\author{
UNIVERSIDADE DE SÃO PAULO \\ FACULDADE DE MEDICINA DE RIBEIRÃO PRETO
}

DÉBORA RIBEIRO CAMPOS

Estudo randomizado sobre a associação da eletroestimulação neuromuscular à mobilização precoce em pacientes críticos de uma unidade de terapia intensiva

Ribeirão Preto 
DÉBORA RIBEIRO CAMPOS

\section{Estudo randomizado sobre a associação da eletroestimulação neuromuscular à mobilização precoce em pacientes críticos de uma unidade de terapia intensiva}

\section{Versão Corrigida}

Tese de doutorado apresentada pela aluna Débora Ribeiro Campos à Faculdade de Medicina de Ribeirão Preto da Universidade de São Paulo para obtenção do título de doutora em ciências.

Área de concentração: Clínica Médica.

Orientador: Prof. Dr. Marcos de Carvalho Borges.

Ribeirão Preto 
Autorizo a reprodução e divulgação total ou parcial deste trabalho, por qualquer meio convencional ou eletrônico, para fins de estudo e pesquisa, desde que citada a fonte.

Campos, Débora Ribeiro

Estudo randomizado sobre a associação da eletroestimulação neuromuscular à mobilização precoce em pacientes críticos de uma unidade de terapia intensiva, 2021.

107 p. : il. ; $30 \mathrm{~cm}$

Dissertação de Doutorado, apresentada à Faculdade de Medicina de Ribeirão Preto/USP. Área de concentração: Clínica Médica.

Orientador: Borges, Marcos de Carvalho.

1. Unidades de terapia intensiva. 2. Estimulação elétrica. 3. Terapia por exercício. 4 . Tempo de internação 
CAMPOS, D. R. Estudo randomizado sobre a associação da eletroestimulação neuromuscular à mobilização precoce em pacientes críticos de uma unidade de terapia intensiva. 2021. Tese de doutorado em ciências (Clínica Médica). Faculdade de Medicina de Ribeirão Preto, Universidade de São Paulo, Ribeirão Preto, 2021.

Aprovado em:

Banca Examinadora

Prof. Dr./Dra.

Instituição

Julgamento

Prof. Dr./Dra.

Instituição

Julgamento

Prof. Dr./Dra.

Instituição

Julgamento 
Dedico este trabalho à minha mãe e ao meu pai, que me guiaram em minha trajetória acadêmica e pessoal, sempre com muito amor, suporte e carinho. Esta conquista também é de vocês. 


\section{Agradecimentos}

Gostaria de agradecer imensamente à minha família e amigos, pois esta tese de doutorado foi fruto de cinco anos de muito trabalho, nos quais eu tive que superar várias barreiras, tanto internas quanto externas. Nesse sentido, o apoio de vocês foi muito importante para que eu tivesse força para persistir e recomeçar quantas vezes fossem necessárias.

Também gostaria de agradecer à Coordenação de Aperfeiçoamento de Pessoal de Nível Superior - Brasil (CAPES), pelo apoio financeiro concedido por meio do Código de Financiamento 001.

À Jackeline e à Thatiana, que me acompanharam no desenvolvimento do projeto. Sinto-me muito grata em trabalhar com pessoas tão dedicadas e dispostas a fazerem sempre o melhor.

Aos fisioterapeutas da UTI da Unidade de Emergência (UE) do Hospital das Clínicas de Ribeirão Preto, em especial: Andi, Larissa, Vivian, Thatiana, Letícia, Talita, Sabrina, Gabriela, Beatriz e a todos os fisioterapeutas que cobriram plantão neste setor. Muitos de vocês foram abertos ao meu projeto desde a sua idealização em 2016, participando, inclusive, da coleta de dados. Por isso, tenho muita gratidão e grande admiração por todos, bem como pelo serviço de fisioterapia desta UTI.

À Dra. Isabella, minha querida amiga, pós-doutoranda na mesma UTI. Sua presença foi como a de um anjo em minha vida. Nós, muitas vezes, dividimos as mesmas dificuldades, e saber que você estava lá, deu-me força para continuar.

Ao Dr. Bruno e ao Dr. Daniel que além de se disponibilizarem a participar do meu trabalho também me auxiliaram no contato com a equipe médica.

A todos os técnicos de enfermagem, enfermeiros, escriturários, médicos e residentes da UTI da UE que também participaram do projeto e dos treinamentos. Obrigada pela paciência e pelo apoio durante todo o desenvolvimento da minha pesquisa. Infelizmente, são muitas pessoas, quando tentei citar os nomes, percebi que sempre faltava alguém. Então, sintam-se todos abraçados e saibam que me lembro de todos os momentos com muito carinho.

Ao Dr. Wilson, à enfermeira Maria Aline, à fisioterapeuta Rosângela e ao Dr. Pazin, que fazem parte da chefia da UE e que permitiram a realização do meu projeto.

À Tânia e ao Rodrigo da Unidade de Pesquisa Clínica que me auxiliaram na parte burocrática do meu projeto, principalmente em seu início.

A todos os funcionários da UE, incluindo todos da equipe de manutenção que foram muito solícitos durante o desenvolvimento da cadeira de PVC, principalmente o Sr. Clóvis. Ao porteiro, Sr. Antônio, à querida Zezé, que me recebia sempre com um sorriso no elevador, e ao meu querido amigo Sr. Nitamar. Acreditem, nunca me esquecerei de todo o apoio que me deram.

Ao secretário da pós-graduação em clínica médica pelo seu trabalho impecável. Emerson, muito obrigada pela paciência e disposição para sempre fazer dar tudo certo. 
Ao Prof. Dr. Rinaldo Guirro que acreditou no meu projeto, me disponibilizou seus equipamentos e me deu suporte com sua expertise em eletroestimulação. Muito obrigada por ter confiado em mim.

To Prof. Dr. Rik Goselink that received me in Belgium for six months and gave me the opportunity to work with his group and participate on his projects. He also supported us on the development of our article, in which we are submitting to a great peer-reviewed magazine. This experienced was very important for me and made me see the world with different eyes. ${ }^{l}$

Ao Prof. Dr. Marcos Borges, que aceitou participar do projeto e me orientou nos mais variados âmbitos que envolvem a pesquisa clínica. Ele me estimulou a pensar grande e ter objetivos que eu nunca havia imaginado, como fazer um estágio fora do país. Obrigada por tudo.

Por fim, gostaria de agradecer a todos os professores que passaram em minha vida até aqui. Eu admiro muito esta profissão e conduzi toda a minha vida buscando me juntar a esse grupo. Encho-me de alegria ao saber que agora eu posso realizar o sonho de trabalhar como professora e, então, poder cumprir o meu propósito de vida.

\footnotetext{
${ }^{1}$ Ao Prof. Dr. Rik Goselink que me recebeu na Bélgica por seis meses e me deu a oportunidade de trabalhar com seu grupo de pesquisa e participar de seus projetos. Ele também nos apoiou no desenvolvimento de nosso artigo, no qual estamos submetendo a uma grande revista científica. Essa experiência foi muito importante para mim e me fez ver o mundo com outros olhos.
} 


\section{Epígrafe}

"Foi o tempo que dedicaste a tua rosa que a fez tão importante." Antoine de Saint-Exupéry 


\section{Resumo}

CAMPOS, D. R. Estudo randomizado sobre a associação da eletroestimulação neuromuscular à mobilização precoce em pacientes críticos de uma unidade de terapia intensiva. 2021. Tese (Doutorado) - Faculdade de Medicina de Ribeirão Preto, Universidade de São Paulo, Ribeirão Preto, 2021.

Introdução: A mobilização precoce (MP) pode acelerar a recuperação funcional, reduzir o tempo de ventilação mecânica e diminuir o tempo de internação hospitalar e na unidade de terapia intensiva (UTI). A estimulação elétrica neuromuscular (EENM) pode ser aplicada precocemente na UTI a fim de manter a força muscular e o estado funcional. Este estudo avaliou o impacto do uso adicional e precoce da EENM a um protocolo de MP. Material e métodos: Este é um estudo controlado e randomizado, realizado na UTI da Unidade de Emergência do Hospital das Clínicas da Faculdade de Medicina de Ribeirão Preto, Universidade de São Paulo, Brasil. Cento e trinta e nove pacientes consecutivos em ventilação mecânica foram incluídos nas primeiras 48 horas de admissão na UTI. Os pacientes foram divididos em dois grupos: MP e MP+EENM. Ambos receberam diariamente o mesmo protocolo de MP. No grupo MP+EENM, os pacientes receberam o uso adicional de EENM, cinco dias por semana, por 60 minutos, começando nas primeiras 48 horas de admissão até a alta da UTI. Foram avaliados o estado funcional, força muscular, independência funcional, tempo de internação na UTI e hospitalar, incidência de delirium, incidência de fraqueza muscular adquirida na UTI, espessura muscular, dias de ventilação mecânica, mortalidade e qualidade de vida. O desfecho primário foi a avaliação pela Escala do estado funcional em unidade de terapia intensiva (FSS-ICU) na alta da UTI. Resultados: Os pacientes do grupo MP+EENM, em comparação com os que só receberam a MP, apresentaram um estado funcional significativamente melhor no primeiro dia acordado $(\mathrm{p}<0,05)$, na alta da UTI $(\mathrm{p}<0,05)$ e na alta hospitalar $(\mathrm{p}<0,05)$. Eles também se levantaram pela primeira vez mais precocemente durante a internação na UTI $(p<0,05)$ e tiveram um 
menor tempo de internação hospitalar, menor incidência de fraqueza muscular adquirida na UTI e melhor força muscular global no primeiro dia acordado $(\mathrm{p}<0,05)$, na alta da UTI $(\mathrm{p}<0,05)$ e na alta hospitalar $(\mathrm{p}<0,05)$. Os dias em ventilação mecânica, a qualidade de vida e a incidência de delirium não mudaram significativamente entre os grupos. Conclusões: A aplicação adicional da EENM precoce a um protocolo de MP promoveu melhores resultados funcionais no primeiro dia acordado, na alta da UTI e na alta hospitalar. Os pacientes que receberam a EENM também levaram menos dias para ficar em pé, tiveram menor tempo de internação hospitalar, menor incidência de fraqueza muscular adquirida na UTI e melhor força muscular nos três momentos avaliados. Estudos futuros ainda são necessários para esclarecer os efeitos de terapias associadas à MP, principalmente para avaliar os desfechos em longo prazo.

Palavras-chave: Unidades de terapia intensiva. Estimulação elétrica. Terapia por exercício. Tempo de internação. 


\begin{abstract}
CAMPOS, D. R. Randomized trial on the association of neuromuscular electrostimulation with early mobilization in critically ill patients of an intensive care unit. 2021. Tese (Doutorado) - Faculdade de Medicina de Ribeirão Preto, Universidade de São Paulo, Ribeirão Preto, 2021.
\end{abstract}

Introduction: Early mobilization (EM) can accelerate functional recovery, reduce the duration of mechanical ventilation and decrease intensive care unit (ICU) and hospital length of stay (LOS). Neuromuscular electrical stimulation (NMES) can be applied earlier during ICU stay to maintain muscle strength and functional status. This study evaluated the impact of the additional use of early NMES to an EM protocol. Methods: This is a randomized controlled trial that took place on the ICUs of the Emergency Unit from the Clinical Hospital of Ribeirão Preto, University of São Paulo, Brazil. One hundred and thirty-nine consecutive mechanical ventilated patients were included in the first 48 hours of ICU admission. The patients were divided into two groups: EM and EM+NMES. Both groups received EM daily. In the EM+NMES group, patients received additionally NMES five days a week, per 60 minutes, starting on the first 48 hours of ICU admission until ICU discharge. Functional status, muscle strength, functional independence, ICU and hospital LOS, incidence of delirium, incidence of ICU-acquired weakness, muscle thickness, days on mechanical ventilation, mortality and quality of life were assessed. Results: Patients in the EM+NMES group presented a significant better functional status on the first day awake, at ICU and hospital discharge and took less days to stand up for the first time during the ICU stay. The EM+NMES group also had a significant shorter hospital LOS, lower incidence of ICUacquired weakness and better global muscle strength at the first day awake, at ICU and hospital discharge. Days on mechanical ventilation, quality of life and incidence of delirium did not significantly change between groups. Conclusions: The additional application of early NMES promoted better functional outcomes on the first day awake and at ICU and hospital discharge. The patients on the EM+NMES group also took fewer days to stand up, had shorter hospital LOS, lower incidence of ICU-acquired weakness and better muscle strength. Future studies are still necessary to clarify the effects of associated therapies to EM, specially to assess long-term outcomes.

Key words: Intensive care units. Electrical stimulation. Exercise therapy. Length of stay. 


\section{Sumário}

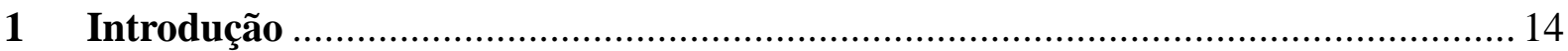

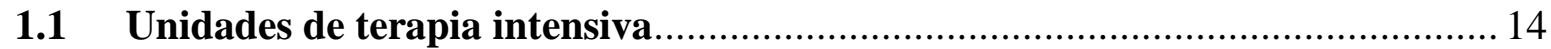

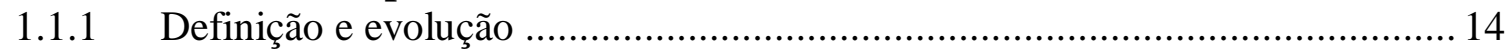

1.1.2 Preditores prognósticos ........................................................................ 15

1.1.3 Consequências da internação na UTI.............................................................. 16

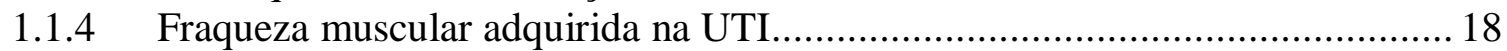

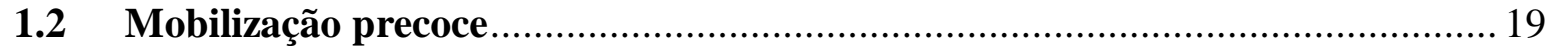

1.2.1 Definição e impactos clínicos...................................................................... 19

1.2.2 Função física e escalas de funcionalidade....................................................... 21

1.2.3 Barreiras à mobilização precoce............................................................... 22

1.3 Estimulação elétrica neuromuscular .............................................................. 23

1.3.1 Definição, parâmetros e características........................................................... 23

1.3.2 Diferenças entre a contração muscular voluntária e a contração causada por

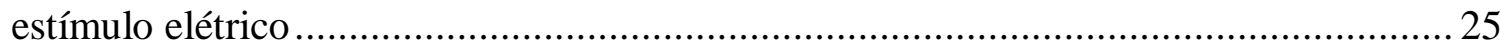

1.3.3 Impactos clínicos da EENM.................................................................... 26

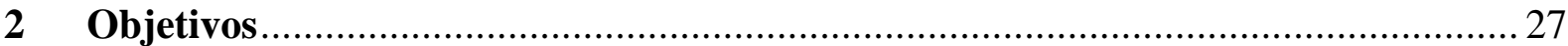

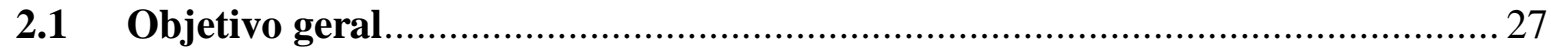

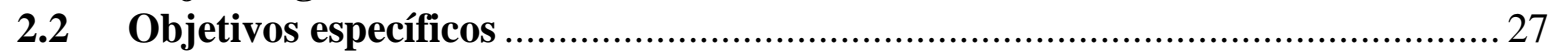

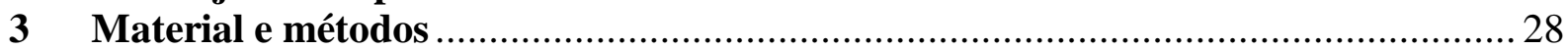

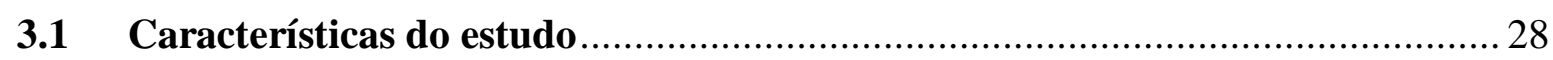

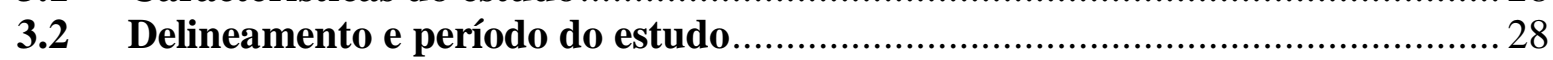

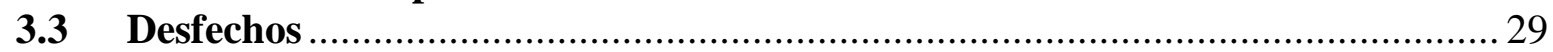

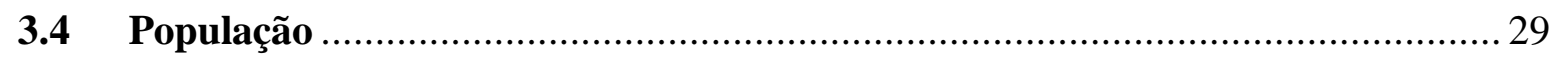

3.5 Grupo multiprofissional de MP.................................................................. 30

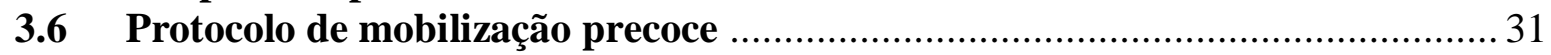

3.7 Protocolo da estimulação elétrica neuromuscular …………………………........ 34

3.8 Critérios de interrupção da aplicação dos protocolos ………………………....... 36

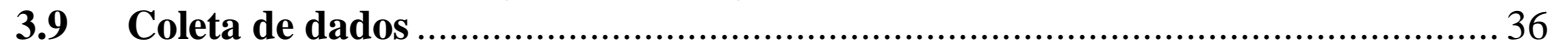

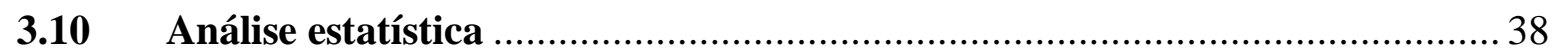

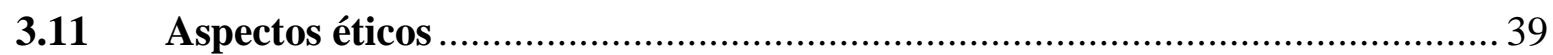

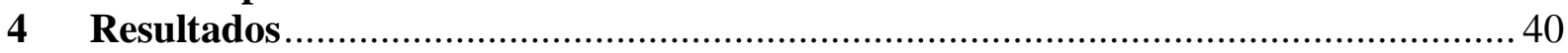

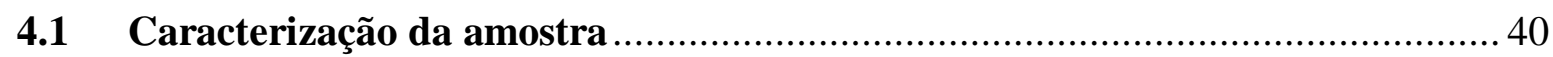

4.2 Características dos protocolos de MP e EENM ………….............................. 42

4.3 Tamanho da amostra de acordo com o desfecho analisado................................. 43

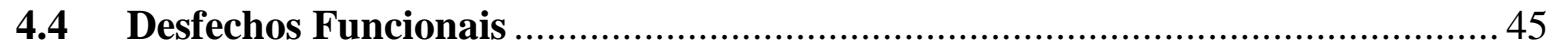

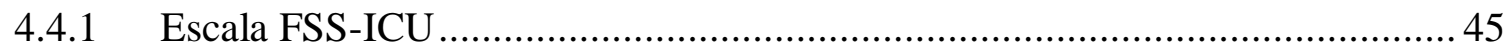

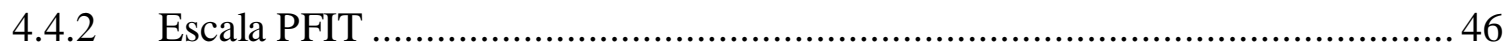

4.4.3 Avaliação da força muscular e incidência de ICU-AW ...................................... 47

4.4.4 Avalição do estado de independência funcional ............................................ 49

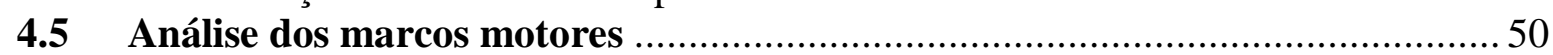

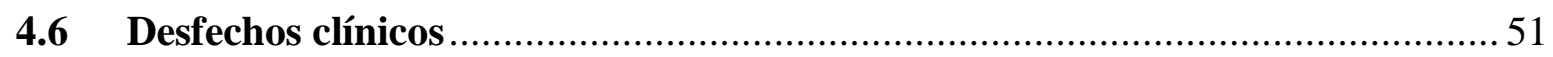

4.6.1 Mortalidade e tempo de ventilação mecânica ………………………………......51

4.6.2 Tempo de internação na UTI e hospitalar .........................................................52

4.6.3 Ultrassom de quadríceps ..................................................................................5 53

4.7 Avaliação da qualidade de vida e da incidência de delirium …………………...54

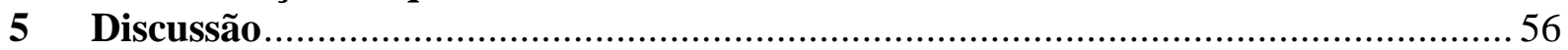

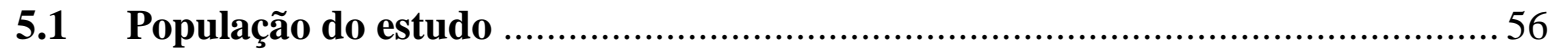

5.2 Aceitabilidade e aplicabilidade dos protocolos .................................................56 


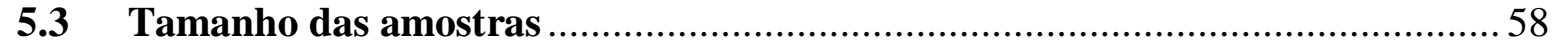

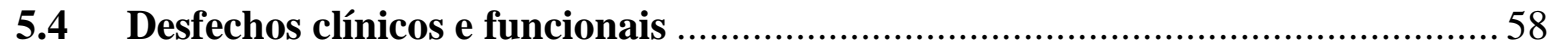

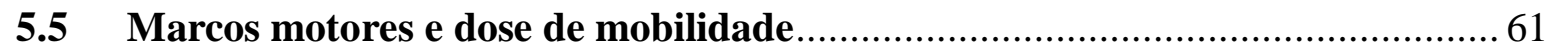

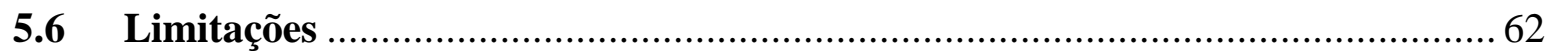

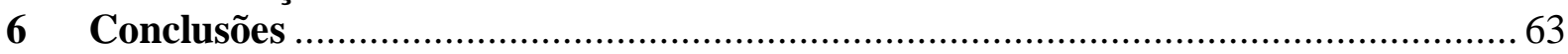

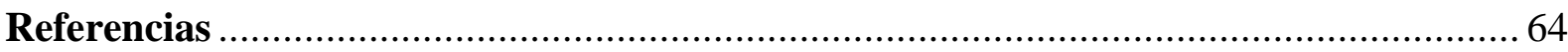

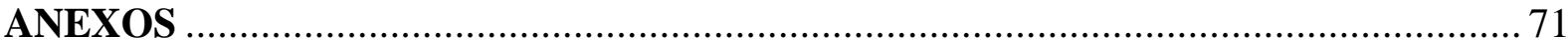

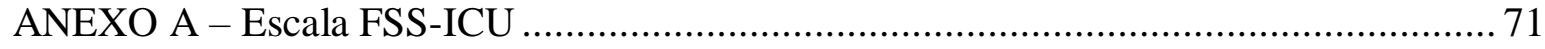

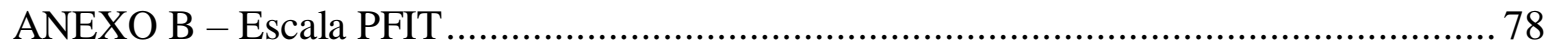

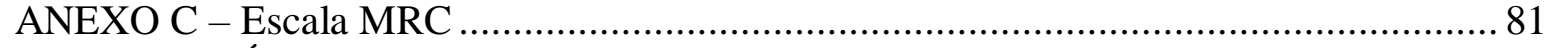

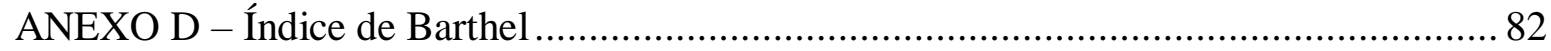

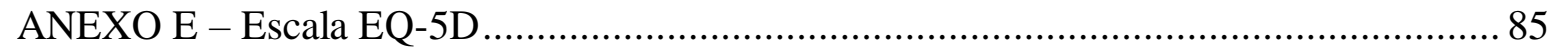

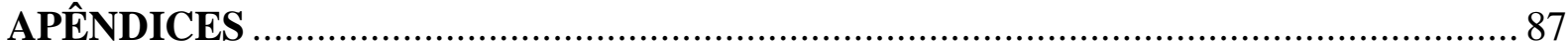

APÊNDICE A - Artigo científico submetido à revista internacional. ............................ 87 


\section{Introdução}

\subsection{Unidades de terapia intensiva}

\subsubsection{Definição e evolução}

Unidades de terapia intensiva (UTIs) podem ser definidas como um ambiente em que profissionais da saúde tratam e observam a evolução clínica de pacientes graves 24 horas por dia. Os pacientes tratados nas UTIs podem apresentar características de todas as especialidades médicas e o objetivo principal é restaurar e manter a função de órgãos vitais, aumentando assim a possibilidade de sobrevida ${ }^{(1)}$. A primeira UTI teve o seu surgimento decorrente da epidemia de poliomielite de 1953 em Copenhagen ${ }^{(1-3)}$. A fim de lidar com este evento impactante, houve a criação de uma sucessão de métodos e soluções não convencionais improvisados às pressas por um hospital dinamarquês que gerou desafios médicos e organizacionais. Isto levou à criação de uma unidade médica especializada para tratar pacientes com poliomielite que, posteriormente, se transformou em uma sala de recuperação com a participação de diversos profissionais, se tornando uma unidade de tratamento intensivo multidisciplinar. Este processo levou 17 meses e foi coordenado pelo médico Dr. Björn Ibsen, considerado o "pai da medicina intensiva"(3).

Apesar de a primeira UTI ter sido criada formalmente em 1952, já no ano de 1850, a enfermeira Florence Nightingale inovou ao criar uma área separada próxima ao posto de enfermagem para soldados britânicos feridos em batalha durante a guerra, especialmente para cuidados após grandes ferimentos de batalha e intervenções cirúrgicas. Isto é frequentemente citado como o verdadeiro começo da medicina intensiva ${ }^{(2,4)}$. Mais uma vez, fica evidente que esta especialidade necessita de um protocolo organizacional envolvendo habilidades de gerenciamento para estruturar e manter um tratamento especializado a pacientes críticos ${ }^{(3)}$.

Inicialmente, embora a maioria das UTIs gerais tenha admitido pacientes clínicos e cirúrgicos, elas tendem a priorizar certos perfis de pacientes com base na natureza do hospital e de acordo com as especialidades das equipes multiprofissionais disponíveis ${ }^{(4)}$. O conceito de UTIs especializadas para atendimento de pacientes no pós-operatório foi rapidamente expandido, especialmente durante a Segunda Guerra Mundial, focadas no tratamento inicial dos pacientes gravemente feridos. O foco era prover intervenções que salvassem vidas baseadas principalmente no controle de sinais vitais ${ }^{(2)}$. 
Além disso, a medicina intensiva está diretamente ligada à necessidade de prover suporte ventilatório, por isso o desenvolvimento da ventilação com pressão positiva foi de extrema importância para o estabelecimento e desenvolvimento da terapia intensiva respiratória ${ }^{(4)}$. Em 1902 foi desenvolvido o primeiro aparato respiratório automático ${ }^{(4)} \mathrm{e}$ agora, mais de 100 anos depois, houve um considerável progresso no desenvolvimento de respiradores modernos, no surgimento da ventilação não-invasiva e até mesmo na evolução da monitorização respiratória ${ }^{(5)}$. Estes desenvolvimentos, além de facilitarem a detecção precoce de interações adversas entre o ventilador e o paciente, aumentaram o conforto do paciente durante a ventilação ${ }^{(6)}$.

Outros fatores envolvidos na medicina intensiva e que tiveram importante progresso nos últimos anos são: tratamento e prevenção de infecções e sepse, monitorização hemodinâmica e cardiovascular, terapias trombolíticas, ressuscitação cardiopulmonar, controle metabólico, manejo do paciente politraumatizado, manejo nutricional, monitorização neurológica, manejo da falência renal e hepática, administração de fluidos e transfusão sanguínea. ${ }^{(5)}$ Baseado nestes fatores e em conjunto com os próprios pacientes ou com a família, os profissionais de saúde devem tomar decisões sobre quais tratamentos são mais indicados de acordo com as condições clínicas dos pacientes. Sendo assim, a discussão do prognóstico clínico se torna mais uma ferramenta fundamental ${ }^{(7)}$.

\subsubsection{Preditores prognósticos}

Apesar de, muitas vezes, o estabelecimento de prognósticos e a tomada de decisões da equipe médica serem baseados na experiência clínica, é importante que se façam estimativas com cálculos numéricos para se avaliar a severidade do quadro clínico ${ }^{(8)}$. Na década de 1970 foram criados alguns modelos de cálculo de predição de prognóstico e de 1979 a 1983 foi desenvolvido um dos instrumentos mais utilizados atualmente, o acute physiology and chronic health evaluation $(\mathrm{APACHE})^{(9,10)}$.

O APACHE I é composto por 34 medidas fisiológicas ${ }^{(9)}$, porém, considerando que naquele tempo a coleta de dados era exclusivamente manual, foi evidenciada a necessidade da criação de um escore prognóstico mais simplificado. Sendo assim, Knaus e colaborados desenvolveram o APACHE II $^{(11)}$. Este escore é mais simples e amplamente utilizado desde 1985. O número de medidas fisiológicas foi reduzido de 34 para 12. Posteriormente foram desenvolvidos o APACHE III e o APACHE IV ${ }^{(10)}$. 
Em 1987, foi criado o Índice de Charlson (Charlson index) com o objetivo de classificar as comorbidades que pudessem alterar o risco de mortalidade, originalmente para uso em estudos longitudinais ${ }^{(12)}$. O índice contém 20 condições clínicas como infarto, insuficiência cardíaca, doença cerebrovascular, úlcera, diabetes, hemiplegia, linfoma, leucemia, doença do fígado, doença renal, dentre outros e considera a idade do paciente, separando-o em 6 grupos. Estes itens recebem pontuações diferentes de acordo com o peso estipulado. A pontuação final é somada e por meio dela constata-se a probabilidade de óbito $^{(13)}$.

Com a finalidade de simplificar as medidas de gravidade, foi também desenvolvido o Simplified Acute Physiology Score 1 (SAPS 1) ${ }^{(10,14)}$. Este escore foi criado em 1984 e, a partir de 14 itens com medidas fisiológicas e variáveis clínicas, reflete o risco de morte dos pacientes internados na UTI. O SAPS 1 tem o objetivo de facilitar os estudos multicêntricos e disponibilizar prognósticos e comparações confiáveis entre grupos de pacientes. As variáveis devem ser coletadas em até 24 horas de admissão na UTI ${ }^{(14)}$. Nos anos de 1990 foi desenvolvido o SAPS 2 com pequenas alterações e em 2005, pesquisadores europeus desenvolveram o SAPS $3 .^{(10,15)}$

Adicionalmente, foi criado o escore de avaliação sequencial de falência de órgãos (SOFA) - Sequential Organ Failure Assessment score - que fornece uma pontuação baseada na avaliação de seis sistemas diferentes: respiratório, cardiovascular, hepático, de coagulação, renal e neurológico ${ }^{(16,17)}$. Este escore se tornou essencial na avaliação de falências de órgãos em pacientes sépticos ${ }^{(16)} \mathrm{e}$, atualmente, é amplamente utilizado para avaliação de pacientes críticos $^{(18)}$.

Considerando que os modelos preditores de prognóstico da UTI já passaram por vários ciclos, espera-se que a próxima geração de modelos preditivos apresentem ainda mais precisão e também incluam medidas de recursos disponíveis e dados financeiros ${ }^{(10)}$.

\subsubsection{Consequências da internação na UTI}

A UTI é um setor complexo, que demanda um trabalho multiprofissional somado à aplicação de técnicas e procedimentos clínicos e cirúrgicos que visam aumentar a sobrevida do paciente ${ }^{(3)}$. Durante a internação na UTI, a causa da internação e as comorbidades prévias associadas à dor e à ansiedade contribuem para uma resposta de estresse que inclui aumento da atividade endógena da catecolaminas, aumento do consumo de oxigênio, taquicardia, hipercoagulabilidade, hipermetabolismo e imunossupressão(19, 20). Portanto, analgesia e 
sedação são administradas para proporcionar conforto ao paciente e garantir sua segurança enquanto diminui a resposta ao estresse e possibilita o foco no tratamento ${ }^{(19,20)}$. No entanto, a sedação em excesso está associada a maior tempo de ventilação mecânica, aumento dos dias de internação na UTI, maior necessidade de avaliações radiológicas do estado mental, maior probabilidade de desenvolver disfunção cerebral e delirium ${ }^{(21)}$. Assim, é necessário equilibrar a necessidade e os benefícios da farmacoterapia sedativa ${ }^{(22)}$.

Adicionalmente, o paciente crítico sofre muitas alterações metabólicas que são resultado de uma complexa combinação de mecanismos e podem levar a múltiplas mudanças nas funções dos órgãos. A resposta metabólica ao estresse envolve um componente neuroendócrino e um componente inflamatório/imune. Quando um estressor é detectado e sinalizado ao sistema nervoso central, uma resposta será acionada, resultando na ativação do sistema nervoso simpático (SNS), do eixo hipotalâmico-hipófise e, posteriormente, afeta processos inflamatórios, imunes e comportamentais. Sendo assim, as intervenções terapêuticas precisam levar em conta a complexidade e os padrões sequenciais da resposta metabólica às doenças críticas ${ }^{(23)}$.

Além disso, devido a sua complexidade clínica, o paciente crítico pode apresentar imobilidade prolongada no leito ocasionando consequências como, por exemplo: alterações metabólicas e inflamação sistêmica, fraqueza muscular, atelectasias, alterações na função vascular, contraturas articulares, tromboembolismo e úlceras de pressão ${ }^{(24)}$. O estudo de Gruther et al. $(2008)^{(25)}$ aponta que a perda de massa muscular aguda apresenta uma correlação negativa com o tempo de internação e parece ser maior nas primeiras 2-3 semanas de imobilização/internação na UTI. Estas consequências podem permanecer após a alta da UTI, ocasionando uma piora da qualidade de $\operatorname{vida}^{(26,27)}$.

Um estudo que explorou as condições reportadas pelos próprios pacientes 4 meses após a alta hospitalar demonstrou que os sintomas mais comumente reportados foram: distúrbios do sono, fadiga, fraqueza e dor ${ }^{(26)}$. Pacientes sobreviventes da síndrome do desconforto respiratório agudo (SDRA) apresentam risco para doenças físicas e neuropsicológicas devido a complicações da própria lesão pulmonar, disfunção multiorgânica e longa permanência na UTI $^{(27)}$. Mesmo um ano após a alta da UTI, estes pacientes podem apresentar incapacidade funcional persistente, perda de massa muscular e fraqueza, enquanto que apenas $49 \%$ retornam ao trabalho ${ }^{(27)}$. Ainda não se sabe quanto tempo leva para que esses pacientes recuperem completamente ou até mesmo se a recuperação completa é realmente possível em todos os $\operatorname{casos}^{(27)}$. 
Outro estudo prospectivo, longitudinal e multicêntrico avaliou pacientes sobreviventes de SDRA e demonstrou que 38\% destes apresentavam fraqueza muscular na alta hospitalar e que uma melhor força muscular na alta pode estar associado a melhor sobrevida nos 5 anos subsequentes ${ }^{(28)}$. Percebe-se que a fraqueza muscular é um problema frequente entre os pacientes críticos que pode ser causada por condições musculares primárias ou por uma desordem secundária ${ }^{(29)}$.

\subsubsection{Fraqueza muscular adquirida na UTI}

Quando a fraqueza muscular se desenvolve como um distúrbio secundário, ela é denominada como fraqueza muscular adquirida na UTI, do inglês, intensive care unit acquired weakness (ICU-AW) ${ }^{(30)}$. A fraqueza adquirida na UTI é normalmente generalizada, simétrica e afeta todos os membros (com predominância nos membros proximais) e músculos respiratórios, enquanto que músculos faciais e oculares são poupados ${ }^{(29,30)}$. O tônus muscular é quase invariavelmente reduzido e os reflexos profundos dos tendões podem ser reduzidos ou normais. A fraqueza pode se originar de um distúrbio neurogênico, denominado de "polineuropatia do doente crítico", de um distúrbio miogênico denominado de "miopatia do doente crítico" ou uma combinação dos dois, nomeado como "polineuromiopatia do doente crítico" $"(29,31)$.

$\mathrm{Na}$ ICU-AW, o exame eletrofisiológico mostra padrões típicos de anormalidades e há uma pronunciada perda de massa muscular, que pode exceder $10 \%$ durante a $1^{\mathrm{a}}$ semana na UTI ${ }^{(32)}$. O estudo de Santos e colaboradores $(2016)^{(33)}$ sugere que a ICU-AW persistente por 6 meses após a resolução da doença crítica está associada a uma fisiopatologia muscular diferente da disfunção aguda. Enquanto que os possíveis mecanismos responsáveis pela perda de massa e função muscular agudas do paciente crítico podem ser a degradação proteolítica muscular em combinação com o silenciamento elétrico e o desacoplamento do mecanismo de excitação-contração, a fraqueza muscular a longo prazo deriva das combinações de atrofia muscular e diminuição da força que podem ocorrer com a ultraestrutura muscular intacta e a função nervosa preservada ${ }^{(33)}$.

A doença crítica, portanto, precisa ser vista como uma sequência contínua de eventos interligados desde os primeiros momentos da internação na UTI, passando pela recuperação até a alta hospitalar e a reabilitação ${ }^{(34)}$. O manejo pré-hospitalar rápido e apropriado para o gerenciamento de pacientes com uma colaboração eficaz entre os serviços de emergência é essencial para otimizar os resultados clínicos. Uma vez internado na UTI, 
um tratamento de sucesso deve envolver não só a sobrevivência do paciente, mas também a sua qualidade de vida ${ }^{(34)}$. Após todo este processo, a decisão de alta de um paciente da UTI deve ser feita com cuidado, pois a alta prematura está associada readmissão na UTI e, possivelmente, com aumento da mortalidade ${ }^{(35)}$.

Assim, considerando os desafios que acompanham a recuperação do doente crítico mesmo após a alta hospitalar, é fundamental expandir as avaliações e intervenções além do ambiente hospitalar, em uma tentativa de melhorar os desfechos físicos e psicológicos dos sobreviventes e de seus familiares cuidadores. Neste contexto, a prevenção tem um importante papel, como com os programas de mobilização precoce em pacientes críticos, que são viáveis, seguros e podem favorecer melhores desfechos $\operatorname{clínicos}^{(24)}$.

\subsection{Mobilização precoce}

\subsubsection{Definição e impactos clínicos}

Ainda não existe um consenso sobre a definição de mobilização precoce (MP). O do significado de "precoce" pode se referir a horas, dias, semanas, ou até mesmo meses ${ }^{(36)}$. Alguns trabalhos definem como "precoce" o primeiro momento em que o paciente se apresenta estável hemodinamicamente, considerando os sistemas respiratório, neurológico e circulatório $^{(37)}$. Uma meta-análise sugere que o melhor momento para se iniciar a MP seria entre 48 a 72 horas da instituição da ventilação mecânica ${ }^{(38)}$.

A definição de "mobilização" pode se referir à movimentação de articulações, membros e até mesmo, pessoas ${ }^{(36)}$. Em alguns casos, a mobilização pode ser usada para descrever um protocolo de tarefas específicas para ficar em pé e caminhar, ministrada pelos profissionais de saúde com aplicação durante toda a internação hospitalar. Em outros casos, mobilização refere-se simplesmente à movimentação de membros dos pacientes no leito ou sentá-los fora da cama ${ }^{(36)}$.

Um programa de MP intensivo pode conter técnicas para respiração adequada durante o exercício, amplitude progressiva de movimento, exercícios enfatizando o fortalecimento muscular, exercícios projetados para melhorar a mobilidade e força do core e exercícios de mobilidade funcional, incluindo mobilidade no leito, transferências, treino de marcha e equilíbrio ${ }^{(39)}$. O seu início pode ser a partir do momento em que o paciente apresente estabilidade hemodinâmica e perdurar durante toda internação na UTI, com o 
objetivo de melhorar a função física até que a maioria dos pacientes possa deambular e realizar atividades de vida diária. Isto torna a MP uma terapia ideal para tratar complicações neuromusculares das doenças críticas ${ }^{(37)}$.

A MP se mostrou aplicável e segura em pacientes críticos, com uma grande variedade de estudos clínicos realizados. Alguns estudos contemplaram pacientes com vários diagnósticos primários como sepse, hemorragias, doença pulmonar obstrutiva crônica (DPOC), lesão pulmonar aguda (LPA), exacerbação de asma e câncer, apresentando como fator de inclusão um tempo de ventilação mecânica (VM) de pelo menos 72 horas $^{(40)}$. Há também estudos realizados especificamente com pacientes diagnosticados com insuficiência

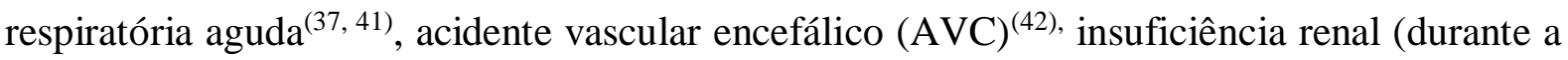
sessão de hemodiálise $)^{(43)}$ e pacientes críticos cirúrgicos ${ }^{(44)}$. Alguns estudos também avaliaram a associação de equipamentos nos protocolos de MP, como, por exemplo, o uso de cicloergômetro $^{(45,46)}$.

Um protocolo de mobilidade aplicado por uma equipe multiprofissional iniciado nas primeiras 48 horas em que o paciente está sob o uso de ventilação mecânica (VM) e nas primeiras 72 horas de internação na UTI pode ser viável, seguro e não aumenta os custos hospitalares ${ }^{(41)}$. Além disso, está associado à diminuição do tempo de permanência na UTI e hospitalar em comparação com pacientes que receberam os cuidados habituais ${ }^{(41)}$.

Uma estratégia para a reabilitação de todo o corpo, que consiste na interrupção diária da sedação e na aplicação de fisioterapia e terapia ocupacional nos primeiros dias de doenças críticas, se mostrou segura e bem tolerada e resultou em melhores desfechos funcionais na alta hospitalar, diminuição nos dias de delirium e diminuição do tempo de ventilação mecânica quando comparados com o tratamento convencional ${ }^{(40)}$.

Outro estudo realizado com pacientes ventilados mecanicamente, usando um processo de controle de qualidade estruturado e multifacetado, reduziu acentuadamente o uso de sedação profunda e aumentou as atividades de MP. Através dessas atividades, foi observada uma diminuição na incidência de delirium na UTI, um aumento na mobilidade funcional, além de uma diminuição no tempo de internação hospitalar ${ }^{(47)}$.

Quanto aos pacientes cirúrgicos, a MP com objetivos direcionados, utilizando-se de um algoritmo validado combinado com o aprimoramento da comunicação interprofissional, melhorou o nível de mobilidade e o tempo de permanência na UTI e aprimorou o desfecho funcional desses pacientes na alta hospitalar ${ }^{(44)}$. Apesar disso, ainda são necessários o desenvolvimento de mais estudos com terapias adicionais à MP para avaliar 
a influência nos desfechos clínicos e possibilitar o estabelecimento de protocolos mais detalhados quanto à frequência, tempo de aplicação e intensidade.

\subsubsection{Função física e escalas de funcionalidade}

A função física, estado funcional, status funcional ou funcionalidade refere-se à capacidade de se realizar várias atividades que vão de autocuidado até atividades mais vigorosas e que exigem graus crescentes de mobilidade, força ou resistência ${ }^{(48)}$. A fim de avaliar os efeitos da MP na função física de pacientes internados em UTI, é importante que se utilize instrumentos específicos ${ }^{(49)}$.

Existem 26 instrumentos para avaliar o status funcional em sobreviventes de UTI, no entanto apenas 6 foram desenvolvidos especificamente para este fim e submetidos à avaliação clinimétrica, que corresponde à aplicabilidade, à capacidade de obter respostas de acordo com o que se deseja avaliar e à capacidade de prever um desfecho clínico ${ }^{(50)}$. Estas seis escalas são: Teste de Função Física em Terapia Intensiva (PFIT) ${ }^{(51)}$, Chelsea Critical Care Physical Assessment tool $(\mathrm{CPAx})^{(52)}$, Escala de mobilidade Perme ${ }^{(53)}$, Surgical intensive care unit Optimal Mobilization Score (SOMS) ${ }^{(54)}$, Escala de Mobilidade na UTI, em inglês ICU Mobility Scale (IMS) ${ }^{(54,55)}$ e a Functional Status Score for the ICU (FSS-ICU) ${ }^{(56,57)}$.

As ferramentas PFIT e CPAx têm as propriedades clinimétricas mais estabelecidas em termos de confiabilidade, validade e capacidade de resposta ${ }^{(49)}$. Parry e colaboradores (2015) apontam que existe uma excelente validade de critério quando comparadas as escalas FSS-ICU e IMS à PFIT no ambiente da UTI. Ainda segundo o estudo, as escalas PFIT e FSS-ICU são as medidas funcionais mais promissoras e devem ser empregadas ao medir a função física na UTI, tanto na prática clínica quanto na pesquisa ${ }^{(49)}$.

A escala PFIT envolve avaliação da força muscular periférica (flexão do ombro e extensão do joelho), sentar-se para prestar assistência e marcha estacionária ${ }^{(51)}$. Ela pode ser usada para orientar a prescrição de exercícios dentro da UTI além de medir a recuperação funcional $^{(51)}$. A FSS-ICU examina um nível hierárquico de dependência/assistência necessária para executar tarefas funcionais de mobilidade no leito, sentado, em pé e andando $^{(49,57)}$. 


\subsubsection{Barreiras à mobilização precoce}

A implementação de um protocolo de MP como parte dos cuidados clínicos de rotina em uma UTI pode ser um desafio. Há uma variedade de barreiras que podem interferir neste processo $^{(58)}$ (tabela $\mathbf{1}$ ).

Tabela 1: Barreiras relacionas à mobilização precoce.

\begin{tabular}{|c|c|}
\hline Tipos de barreiras & \\
\hline $\begin{array}{l}\text { Barreiras relacionadas ao paciente: } \\
\text { sintomas e condições clínicas }\end{array}$ & $\begin{array}{l}\text { Gravidade da doença } \\
\text { Instabilidade hemodinâmica/arritmias } \\
\text { Instabilidade respiratória/assincronia com a VM } \\
\text { Dor } \\
\text { Mal estado nutricional ou obesidade } \\
\text { Fraqueza prévia } \\
\text { Sedação profunda ou paralisia } \\
\text { Delirium ou agitação } \\
\text { Recusa do paciente, falta de motivação, ansiedade } \\
\text { Fadiga, necessidade de descanso, sonolência } \\
\text { Cuidados paliativos } \\
\text { Dispositivos de monitorização }\end{array}$ \\
\hline $\begin{array}{l}\text { Barreiras estruturais: } \\
\text { falta de recursos humanos e/ou técnicos }\end{array}$ & $\begin{array}{l}\text { Número de profissionais limitado e limitação de tempo } \\
\text { Falta de um programa ou protocolo de MP } \\
\text { Falta de equipamentos } \\
\text { Alta precoce da UTI (antes de ser realizado mobilização) }\end{array}$ \\
\hline Barreiras relacionadas à cultura da UTI & $\begin{array}{l}\text { Falta de uma cultura voltada à mobilidade } \\
\text { Falta de uma cultura multidisciplinar } \\
\text { Falta de conhecimento da equipe sobre os riscos e benefícios da } \\
\text { MP } \\
\text { A MP não ser considerada uma prioridade } \\
\text { Falta de conhecimento sobre MP pela família ou pelo paciente }\end{array}$ \\
\hline Barreiras relacionadas a processos & $\begin{array}{l}\text { Falta de planejamento e coordenação } \\
\text { Falta de clareza nas responsabilidades de cada funcionário } \\
\text { Riscos que a MP gera, como stress e lesões } \\
\text { Manutenção da ordem de repouso no leito desnecessariamente } \\
\text { Falta de uma checagem diária para avaliar elegibilidade para MP }\end{array}$ \\
\hline
\end{tabular}

Fonte: Adaptado de Dubb et. al (2016) ${ }^{(58)}$.

Esforços sistemáticos para mudar cultura da UTI visando priorizar a MP e usando uma abordagem interprofissional, com a criação de estratégias que vençam cada uma destas 
barreiras, são importantes componentes da implementação bem-sucedida da MP na prática clínica $^{(58)}$.

É fato que uma importante razão para a inconsistência observada na aplicação da MP em pacientes de UTI é a falta de uma abordagem multiprofissional protocolada e uniforme $^{(41)}$. Por isso, com o objetivo de transpassar algumas destas barreiras, muitos centros têm proposto o uso de protocolos e/ou diretrizes de mobilização precoce, bem como um “Time de mobilização precoce" multiprofissional ${ }^{(41)}$.

\subsection{Estimulação elétrica neuromuscular}

\subsubsection{Definição, parâmetros e características}

A mobilização precoce desempenha um importante papel na prevenção da fraqueza muscular, mas apesar de ser possível realizar mobilizações passivas e mudanças de decúbito em pacientes sedados/inconscientes, não é possível fazer com que eles produzam contrações musculares ativas. Por isso, é importante desenvolver estratégias adicionais para atenuar a atrofia muscular por desuso ${ }^{(59)}$.

A introdução de algum nível de contração muscular pela aplicação da estimulação elétrica neuromuscular (EENM) somada a um suporte nutricional adequado pode aumentar a síntese proteica muscular e prevenir ou atenuar a perda muscular por desuso ${ }^{(59)}$. Uma das vantagens da EENM é que o paciente não necessita estar desperto e consciente para realizar esta terapia, favorecendo a sua utilização mesmo em pacientes sedados.

A EENM ocorre por um impulso elétrico liberado por eletrodos localizados na superfície da pele que promove a ativação de vias intramusculares, causando uma contração muscular visível ${ }^{(60-62)}$. Os estímulos elétricos geralmente são entregues usando um ou mais eletrodos ativos posicionados próximos aos pontos motores musculares e unidades de estimulação pré-programadas. Um pré-requisito para obter contrações musculares com EENM é a presença de um nervo motor intacto ${ }^{(60)}$.

Para a aplicação da EENM, devem-se considerar diversos fatores que são divididos em quatro categorias ${ }^{(60)}$, dispostas na tabela 2. 
Tabela 2: Fatores relacionados à eletroestimulação neuromuscular.

\begin{tabular}{ll}
\hline Categorias & Tipo de corrente; \\
\hline Características dos parâmetros utilizados & Frequência em Hz; \\
& Intensidade de corrente em miliampères (mA); \\
& Duração de pulso em microssegundos ( $\mu$ s); \\
& Tempo de tratamento em minutos; \\
& Rampa ou tempo de subida em segundos: tempo que leva \\
& para se atingir os parâmetros previamente designados; \\
& Ciclo de trabalho ou tempo on-off: tempo em que o estímulo \\
& é ligado e desligado. \\
& Duração da contração; \\
& Dose de treinamento; \\
& Comprimento muscular; \\
& Utilização da EENM sozinha ou em conjunto com outra \\
& terapia. \\
\hline Características da contração muscular & Capacidade do aparelho; \\
& Tipo de eletrodo: implantado ou superficial; \\
& Material do eletrodo: silicone ou carbono. \\
\hline Características do aparelho utilizado & Músculo a ser estimulado; \\
& Número de sessões por semana; \\
& Número total de sessões \\
& Tolerância pelo paciente; \\
& Cumprimento do programado. \\
\hline Fogramação de treinamento & \\
& \\
&
\end{tabular}

Fonte: Adaptado de Maffiuletti et. al (2010) ${ }^{(60)}$.

Existe uma grande heterogeneidade nos parâmetros utilizados ${ }^{(63)}$. No entanto, é fortemente recomendado o uso de corrente bifásica retangular, uma duração de pulso de 100$400 \mu$ s e uma frequência de $50-100 \mathrm{~Hz}$ para ativação muscular ${ }^{(60,63,64)}$.

A intensidade de corrente (mA) é aumentada de acordo com a resposta motora visual no momento da aplicação. A corrente elétrica necessária para uma resposta contrátil geralmente é maior nas extremidades inferiores ${ }^{(65)}$. 


\subsubsection{Diferenças entre a contração muscular voluntária e a contração causada por estímulo elétrico}

As contrações causadas pela EENM são consideravelmente diferentes das contrações de ativação voluntárias. Enquanto nas contrações voluntárias o tempo de recrutamento das unidades motoras é assincrônico, as contrações realizadas pelo aparelho são feitas de forma sincrônica ${ }^{(60)}$. Além disso, a EENM tem um recrutamento espacial de fibras limitado, estimulando as mais superficiais e as mais próximas dos eletrodos ${ }^{(60,64)}$.

A contração muscular provocada por estímulo elétrico pode induzir maiores mudanças metabólicas locais que uma contração muscular voluntária (CMV). Esta característica torna esta técnica um bom adjuvante no tratamento da atrofia do tecido muscular, quando usado em conjunto com a contração muscular ativa ${ }^{(64)}$.

A EENM também pode induzir efeitos sistêmicos benéficos na microcirculação que é um fator relacionado à função endotelial. Desta forma, a aplicação do estímulo elétrico pode mobilizar células progenitoras endoteliais que têm a função de neovascularização, reparo endotelial e restauração do endotélio ${ }^{(66)}$. Paralelamente, devido ao próprio tipo de estímulo elétrico causado pela EENM e à presença de uma maior acidose local que na CMV, fibras musculares do tipo II (rápidas e glicolíticas) são preferencialmente recrutadas durante a EENM. Estas fibras musculares são fisiologicamente constituídas para prover mais potência e força, porém são menos resistentes ${ }^{(64)}$ e a EENM também atua no aumento do tamanho da fibra $^{(63)}$.

Apesar dos efeitos da EENM serem principalmente nos grupos musculares diretamente estimulados, ocorrem também efeitos sistêmicos. A EENM do músculo quadríceps aumenta agudamente a resposta hemodinâmica nas regiões do córtex sensório$\operatorname{motor}^{(67)}$.

Outro fator importante se refere a respondedores e não respondedores à estimulação muscular. Pacientes gravemente enfermos com sepse, edema ou vasopressores são menos propensos a apresentar uma contração adequada do quadríceps sob efeito da EENM, e esta resposta também é mais efetiva no início da permanência na UTI em comparação com uma semana após a internação ${ }^{(61)}$.

Adicionalmente, uma das principais limitações da EENM é a possibilidade de causar desconforto associado à estimulação periférica ${ }^{(60)}$. Esta talvez seja uma importante limitação em pacientes conscientes, mas pode ser um fator menos importante em pacientes comatosos ou sedados. 


\subsubsection{Impactos clínicos da EENM}

Existem protocolos variados e resultados divergentes sobre o uso da EENM e seus impactos clínicos. Há uma prevalência de pesquisas com tamanho amostral pequeno somada a uma falta de estudos com alta qualidade metodológica, o que limita a confiabilidade e comparação dos resultados, especialmente entre intervenções que não utilizam a mesma parametrização. Assim, é necessário o desenvolvimento de mais ensaios clínicos, com o objetivo de estabelecer um padrão para o uso desta terapia, com parâmetros e tempos de estimulação por dia de tratamento bem definidos, de modo que sua eficácia possa ser confirmada, isolada ou em combinação ${ }^{(68,69)}$. Além disso, poucos estudos avaliaram o impacto da EENM na qualidade de vida e em desfechos funcionais ${ }^{(69)}$.

Em pacientes comatosos, a EENM pode ser uma estratégia efetiva para prevenir a atrofia muscular esquelética ${ }^{(70)}$. A aplicação diária da EENM nas extremidades inferiores de pacientes críticos também pode aumentar a espessura da camada muscular ${ }^{(71,}{ }^{72)}$. Em pacientes sépticos, pode aumentar a força muscular ${ }^{(73)}$. Quando aplicada nos músculos vasto lateral, medial e fibular longo em pacientes críticos pode prevenir a instalação da polineuromiopatia do doente crítico, além de diminuir o tempo de ventilação mecânica ${ }^{(74)}$. Em pacientes com doença pulmonar obstrutiva crônica a EENM também melhorou a força muscular e causou um aumento de fibras musculares do tipo $1^{(75)}$.

Quando combinado com um protocolo de exercícios, a EENM também apresentou resultados positivos, em que o grupo que teve a associação das duas técnicas teve uma menor duração do tempo de ventilação mecânica e do tempo de sedação ${ }^{(76)}$. Por outro lado, outros estudos não demonstraram melhores resultados em grupos que foram tratados com EENM associada a um protocolo de mobilização precoce ${ }^{(77,78)}$, a uma plataforma vibratória corporal $^{(77)}$ ou a cicloergômetros ${ }^{(78)}$.

Assim, considerando os benefícios da MP e a facilidade de utilização da EENM em pacientes sedados, nós hipotetizamos que a associação da EENM a um protocolo de MP pode melhorar os desfechos funcionais de pacientes críticos. 


\section{Objetivos}

\subsection{Objetivo geral}

Avaliar se o uso adicional e precoce da EENM a um protocolo de MP, aplicado em pacientes internados em UTI, pode resultar em melhores desfechos clínicos e funcionais.

\subsection{Objetivos específicos}

a) Comparar os efeitos da aplicação da associação de MP e EENM no status funcional, força muscular e independência funcional de pacientes críticos no primeiro dia acordado, na alta da UTI e na alta hospitalar;

b) Comparar os efeitos da aplicação da associação de MP e EENM na mortalidade, tempo de VM, tempo de internação na UTI e hospitalar;

c) Determinar a dose de mobilidade e os marcos motores resultantes do protocolo de MP;

d) Analisar os efeitos da aplicação da EENM nos sinais vitais dos pacientes antes e após as intervenções;

e) Avaliar como a associação de MP e EENM afeta a incidência de delirium durante a internação na UTI e a qualidade de vida dos pacientes na alta da UTI e na alta hospitalar;

f) Detectar as diferenças entre os grupos na incidência de fraqueza muscular adquirida na UTI.

g) Comparar os efeitos da aplicação da associação de MP e EENM na camada muscular de quadríceps; 


\section{Material e métodos}

\subsection{Características do estudo}

O presente estudo se caracteriza como prospectivo, randomizado, unicêntrico. Foi realizado nas UTIs adulto da Unidade de Emergência do Hospital das Clínicas da Faculdade de Medicina de Ribeirão Preto da Universidade de São Paulo (UE-HCFMRP-USP).

A UE-HCFMRP-USP é um hospital exclusivo para o atendimento de urgências e emergências, sendo referência terciária para 26 municípios da Divisão Regional de Saúde XIII do Estado de São Paulo (Ribeirão Preto) que totaliza, aproximadamente, 1.200.000 habitantes. Apresenta uma média de internação anual de aproximadamente 20.000 pacientes. Ela atende exclusivamente pacientes do Sistema Único de Saúde (SUS) e é vinculada à FMRP-USP, o que proporciona o desenvolvimento de atividades de ensino, pesquisa e assistência. A UEHCFMRP-USP tem duas UTIs para pacientes adultos clínicos e cirúrgicos, com um total de 18 leitos.

\subsection{Delineamento e período do estudo}

Os pacientes internados nas UTIs adulto da UE-HCFMRP-USP há menos de 48 horas e que não apresentaram nenhum critério de exclusão, foram randomizados entre dois grupos: o grupo em que foi realizada a MP e o grupo em que foi realizada a MP associada à EENM (MP+EENM), com relação de randomização de 1:1. A coleta de dados teve duração de um ano e seis meses, iniciando-se em julho de 2018 com encerramento em dezembro de 2019.

Incialmente, foi criado um grupo multidisciplinar de MP para discutir questões relacionadas ao treinamento da equipe e às barreiras à aplicação dos protocolos. Posteriormente, foi definido o protocolo de MP, que foi aplicado previamente ao início da randomização, de janeiro a junho de 2018 (seis meses). Assim, a MP foi realizada pela equipe assistencial, de maneira semelhante em todos os pacientes. A EENM foi realizada por uma única fisioterapeuta, 5 vezes por semana, desde a randomização até a alta da UTI. Avaliações do status e independência funcional, força muscular e qualidade de vida foram realizadas por meio de escalas específicas durante a internação na UTI e na alta hospitalar pela mesma avaliadora. Incidência de delirium foi avaliada todos os dias pela equipe de enfermagem. E 
por fim, ultrassom de quadríceps na admissão e na alta da UTI foi aplicado pela equipe médica.

A randomização e o armazenamento de dados foram realizados por meio da plataforma Redcap ${ }^{\circledR}$ (Research Electronic Data Capture: project-redcap.org). A lista de randomização foi gerada eletronicamente (www.sealedenvelope.com). O sigilo da lista de randomização foi mantido durante todo o estudo. A fim de obter uma amostra similar em relação à idade em ambos os grupos, foi realizada randomização estratificada por faixa etária: jovens (18-29 anos), adultos (30-59 anos) ou idosos (60 anos ou mais).

\subsection{Desfechos}

a) Desfecho primário:

- Status funcional avaliado pela escala de estado funcional em unidade de terapia intensiva (FSS-ICU) na alta da UTI.

b) Desfechos secundários:

- Status funcional avaliado pelo teste de função física na unidade de terapia intensiva (PFIT);

- Independência funcional e mobilidade;

- Força muscular;

- Incidência de ICU-AW;

- Tempo de internação na UTI;

- Tempo de internação hospitalar;

- Tempo de VM;

- Mortalidade;

- Avaliação de qualidade de vida;

- Incidência de delirium;

- Medida da espessura da camada muscular de quadríceps.

\subsection{População}

a) Critérios de inclusão:

- Pacientes maiores de 18 anos;

- Previsão de tempo de intubação maior ou igual há 48 horas; 
- Possibilidade de inclusão em até 48 horas da admissão na UTI;

- Assinatura do Termo de Consentimento Livre e Esclarecido pelo paciente ou responsável.

b) Critérios de exclusão:

- Inabilidade de andar sem assistência antes da doença aguda que gerou a internação na UTI;

- Doença neuromuscular prévia (tais como, miastemia grave, esclerose lateral amiotrófica);

- Fratura de quadril;

- Trauma raqui-medular;

- Epilepsia como diagnóstico que levou a internação ou crises convulsivas não controladas;

- Risco de óbito em 24 horas;

- Suspeita ou constatação de morte encefálica;

- Presença de marca-passo;

- Condições musculoesqueléticas anormais e/ou condições de pele que poderiam interferir com a eletroestimulação (por exemplo, fratura de fêmur, uso de fixador externo, ferida em membros inferiores, doença de pele ou queimadura na região anterior da coxa).

\subsection{Grupo multiprofissional de MP}

Para uma melhor adesão ao protocolo, foi criado um grupo multiprofissional de MP baseado em estudos prévios ${ }^{(41)}$. O grupo foi composto por dois enfermeiros, dois técnicos de enfermagem, dois fisioterapeutas e dois médicos. Foram realizadas reuniões para discutir questões relacionadas às barreiras encontradas na implantação do protocolo, como por exemplo: manejo de funcionários, melhores horários para realização de mudanças de decúbito, definição de temas para aulas de capacitação para a equipe, entre outros. 


\subsection{Protocolo de mobilização precoce}

Foi realizado um treinamento com toda a equipe das UTIs, um total de 100 funcionários, incluindo técnicos de enfermagem, enfermeiros, médicos, fisioterapeutas e residentes. Eles foram divididos em grupos de 4 a 8 profissionais, em que eram aplicadas instruções específicas de acordo com a profissão e a função na UTI. O treinamento consistia em uma aula inicial, onde foram abordadas as principais consequências da imobilidade, os benefícios da MP, suas indicações e contraindicações e a importância de uma equipe multiprofissional na aplicação de um protocolo de MP. Além disso, os fisioterapeutas foram instruídos a realizar orientações contínuas para os outros profissionais referentes a técnicas de mudança de decúbito e posicionamentos durante a prática clínica. Também foram desenvolvidos treinamentos práticos referentes ao manejo do dispositivo de guincho.

Após este processo, o protocolo de MP foi aplicado por toda a equipe multiprofissional das UTIs, sendo coordenado pelos fisioterapeutas. Havia em média 8 fisioterapeutas nas UTIs, que revezavam entre os turnos matutino, vespertino e noturno (até as 00:00). A fim de trazer uniformidade ao estudo, o protocolo de MP foi instituído previamente ao início da randomização, de janeiro a junho de 2018 (seis meses).

O protocolo de MP utilizado foi desenvolvido especialmente para este estudo e visa à retirada rápida do paciente do leito e de acordo com as recomendações do Departamento de Fisioterapia da Associação de Medicina Intensiva Brasileira ${ }^{(79)}$. O protocolo tem 6 fases designadas de acordo com o nível de consciência do paciente e o que ele tem capacidade de fazer (Tabela 3). Em pacientes inconscientes ou profundamente sedados é realizada a mobilização passiva e, à medida que o nível de consciência aumenta, são realizados exercícios ativos e resistidos. Atividades fora do leito são sempre prioridade. A aplicação do protocolo de MP foi incorporada às práticas assistenciais das UTIs.

Para facilitar o posicionamento dos pacientes na posição sentada no leito, foi desenvolvido, juntamente com a equipe da engenharia clínica e da manutenção da UEHCFMRP-USP, um equipamento com tubos de policloreto de vinila (PVC). O equipamento foi inspirado em protótipos disponíveis online e teve as dimensões adaptadas para o tamanho dos leitos. Foram construídos três protótipos até a concretização da versão final (figura 1).

As sessões de MP têm duração de 20-60 minutos, conforme a realização das atividades que o paciente consegue completar e de acordo com as fases do protocolo. $\mathrm{O}$ paciente não necessariamente precisa passar por todas as fases, ele pode começar em uma fase 
mais avançada, de acordo com a sua condição clínica. O protocolo serve como guia para que a mobilização seja realizada da forma mais completa possível.

Tabela 3 (continua): Protocolo de mobilização precoce: Requisitos de acordo com a fase designada e exercícios a serem realizados.

\begin{tabular}{|c|c|c|}
\hline Fase & Requisitos & Exercícios \\
\hline Fase 1 & $\begin{array}{l}\text { Paciente inconsciente ou } \\
\text { não colaborativo }\end{array}$ & $\begin{array}{l}\text { Avaliar a possibilidade de diminuir a sedação. } \\
\text { Posição sentada no leito (cabeceira de } 45^{\circ} \text { a } 60^{\circ} \text { ) por pelo menos } 30 \text { min } \\
\text { Cabeceira elevada de } 45^{\circ} \text { a } 60^{\circ} \text { durante a fisioterapia } \\
\text { Alongamentos estáticos } \\
\text { Mudança de decúbito a cada duas horas } \\
\text { Mobilização passiva de MMSS e MMII } \\
\text { Ao final do atendimento, posicionamento à beira leito com apoio para } \\
\text { teste de controle de tronco por } 10 \text { minutos e/ou sentado na poltrona com } \\
\text { o guincho por } 30 \text { minutos a } 1 \text { hora, conforme indicação clínica (paciente } \\
\text { estável hemodinamicamente) }\end{array}$ \\
\hline Fase 2 & $\begin{array}{l}\text { Paciente não totalmente } \\
\text { consciente e colaborativo }\end{array}$ & $\begin{array}{l}\text { Posição sentada no leito (cabeceira de } 45^{\circ} \text { a } 60^{\circ} \text { ) por pelo menos } 30 \text { min } \\
\text { Cabeceira elevada de } 45^{\circ} \text { a } 60^{\circ} \text { durante a fisioterapia } \\
\text { Mobilização predominantemente ativo-assistida de MMSS e MMII } \\
\text { Alongamentos estáticos } \\
\text { Mudança de decúbito a cada duas horas } \\
\text { Propriocepção articular } \\
\text { Ao final do atendimento, posicionamento à beira leito com apoio para } \\
\text { teste de controle de tronco por } 10 \text { minutos e/ou sentado na poltrona com } \\
\text { o guincho por } 30 \text { minutos a } 1 \text { hora, conforme indicação clínica (paciente } \\
\text { estável hemodinamicamente) }\end{array}$ \\
\hline Fase 3 & $\begin{array}{l}\text { Paciente consciente e } \\
\text { colaborativo }\end{array}$ & $\begin{array}{l}\text { Posição sentada no leito (cabeceira de } 45^{\circ} \text { a } 60^{\circ} \text { ) por pelo menos } 30 \text { min } \\
\text { Cabeceira elevada de } 45^{\circ} \text { a } 60^{\circ} \text { durante a fisioterapia } \\
\text { Exercícios ativos e/ou ativos resistidos de MMSS e MMII } \\
\text { Alongamentos estáticos } \\
\text { Propriocepção articular } \\
\text { Mobilização aeróbica } \\
\text { Transferência do paciente para a borda da cama (exercícios de controle } \\
\text { de tronco e equilíbrio) e, se possível, posicioná-lo sentado na poltrona ao } \\
\text { fim do atendimento }\end{array}$ \\
\hline
\end{tabular}

MMSS: membros superiores; MMII: Membros inferiores. 
Tabela 3 (conclusão): Protocolo de mobilização precoce: Requisitos de acordo com a fase designada e exercícios a serem realizados.

\begin{tabular}{|c|c|c|}
\hline Fase & Requisitos & Exercícios \\
\hline Fase 4 & $\begin{array}{l}\text { Paciente consciente } \\
\text { e colaborativo }\end{array}$ & $\begin{array}{l}\text { Realizar fisioterapia com o paciente sentado à beira leito ou sentado na } \\
\text { poltrona } \\
\text { Exercícios ativos e/ou ativos resistidos de MMSS e MMII } \\
\text { Alongamentos estáticos } \\
\text { Propriocepção articular } \\
\text { Mobilização aeróbica } \\
\text { Transferência do paciente para a borda da cama (exercícios de controle } \\
\text { de tronco e equilíbrio) } \\
\text { Posicionamento sentado na poltrona com auxílio da equipe ou guincho } \\
\text { uma vez ao dia, por pelo menos } 30 \text { minutos }\end{array}$ \\
\hline Fase 5 & $\begin{array}{l}\text { Paciente consciente } \\
\text { e colaborativo }\end{array}$ & $\begin{array}{l}\text { Realizar fisioterapia com o paciente sentado beira leito, sentado na } \\
\text { poltrona ou em posição ortostática } \\
\text { Exercícios ativos e ativos resistidos de MMSS e MMII } \\
\text { Alongamentos estáticos } \\
\text { Propriocepção articular } \\
\text { Mobilização aeróbica } \\
\text { Transferência do paciente para a borda da cama (exercícios de controle } \\
\text { de tronco e equilíbrio) } \\
\text { Posicionamento sentado na poltrona ativamente ou com auxílio da } \\
\text { equipe por, no mínimo, uma vez ao dia, por pelo menos } 30 \text { minutos } \\
\text { Ortostatismo ativo (exercícios de equilíbrio e marcha estacionária) }\end{array}$ \\
\hline Fase 6 & $\begin{array}{l}\text { Paciente consciente } \\
\text { e colaborativo }\end{array}$ & $\begin{array}{l}\text { Realizar fisioterapia com o paciente em pé ou sentado na poltrona } \\
\text { Exercícios ativos e ativos resistidos de MMSS e MMII } \\
\text { Alongamentos estáticos } \\
\text { Propriocepção articular } \\
\text { Mobilização aeróbica } \\
\text { Posicionamento sentado na poltrona ativamente por, no mínimo, uma } \\
\text { vez ao dia, por pelo menos } 1 \text { hora } \\
\text { Ortostatismo ativo (exercícios de equilíbrio, controle de tronco e marcha } \\
\text { estática) } \\
\text { Deambulação assistida ou independente }\end{array}$ \\
\hline
\end{tabular}

MMSS: membros superiores; MMII: Membros inferiores.

Fonte: autoria própria (2021). 
Figura 1: Fotos demonstrativas do apoio de PVC desenvolvido em conjunto com a equipe de engenharia clínica e manutenção da UE-HCFMRP-USP.

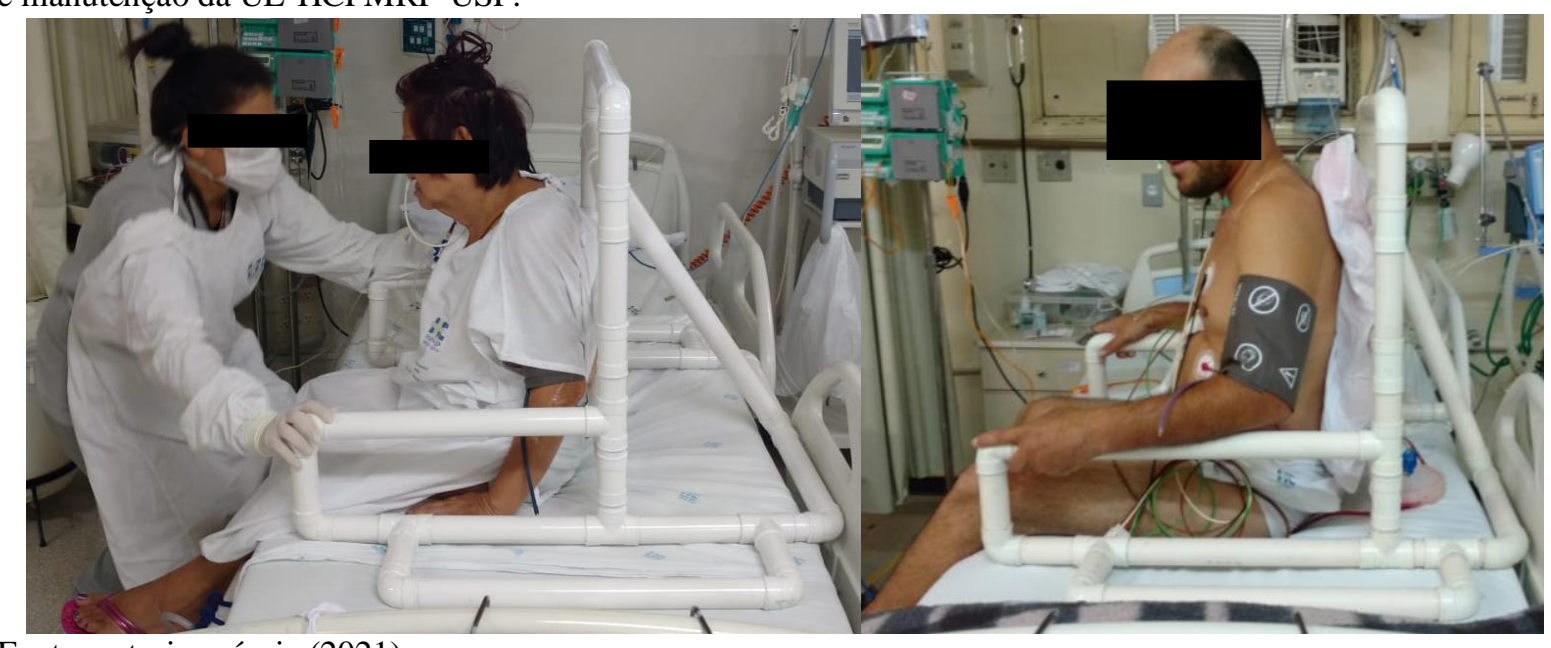

Fonte: autoria própria (2021).

Na grande maioria das situações não é possível classificar a fase exata em que o paciente está no protocolo, pois ele pode apresentar características de duas fases ao mesmo tempo. Como por exemplo, em casos em que há diferença de força e mobilidade entre membros superiores e inferiores ou entre o lado direito e esquerdo. Sendo assim, a fim de classificar e padronizar a dose de mobilidade, foram coletados dados referentes aos marcos motores principais durante a internação na UTI. Estes dados foram coletados por meio das evoluções clínicas diárias da equipe de fisioterapia.

a) Os marcos motores principais são:

- Sentar na beira do leito;

- Sentar na poltrona;

- Ortostatismo;

- Marcha estacionária;

- Deambular.

b) Para cada um destes marcos foram coletadas informações referentes ao:

- Número de pacientes que realizaram a atividade;

- Número de dias de internação até a primeira ocorrência.

\subsection{Protocolo da estimulação elétrica neuromuscular}

O protocolo de EENM foi desenvolvido sob orientação do prof. Dr. Rinaldo Roberto de Jesus Guirro, Departamento de Ciências da Saúde da FMRP-USP. As sessões de EENM foram realizadas exclusivamente pela fisioterapeuta responsável pelo presente estudo, 
apenas nos pacientes do grupo MP+EENM, uma vez por dia, cinco dias por semana desde o primeiro dia de internação até a alta da UTI. O paciente era posicionado em decúbito dorsal, com o tronco elevado de $30^{\circ}$ a $45^{\circ}$ e joelhos estendidos. A EENM era aplicada nos músculos quadríceps femoral e tibial anterior, bilateralmente. Dois eletrodos de silicone-carbono $(8.0 \mathrm{x}$ $4.0 \mathrm{~cm}$ ) eram acoplados transversalmente em cada coxa, sobre os ventres musculares do quadríceps, sendo um proximal e um distal, e outros dois eletrodos de silicone-carbono $(4.0 \mathrm{x}$ $2.0 \mathrm{~cm}$ ) eram acoplados longitudinalmente na região anterior da perna, sobre os ventres musculares do músculo tibial anterior. Um gel hidrossolúvel era aplicado embaixo dos eletrodos e a fixação era feita com fita adesiva, ou com esparadrapo em pacientes mais agitados (figura 2).

Dois aparelhos de EENM modelo DUALPEX®(Quark Equipamentos Médicos, Piracicaba, SP, Brasil) foram utilizados simultaneamente, um para a terapia no músculo quadríceps e o outro para o tibial anterior. Os eletrodos que eram conectados aos aparelhos e ligados simultaneamente, geravam um pulso retangular bifásico e simétrico $(80 \mathrm{~Hz}, 400 \mu \mathrm{s}, 5$ segundos on, 10 segundos off e subida de 1 segundo) por um período de 1 hora.

A intensidade do estímulo era aumentada até o ponto em que houvesse plena contração muscular e em que as fibras estivessem visíveis e/ou palpáveis ${ }^{(80)}$. A máxima intensidade atingida pelo aparelho era $120 \mathrm{~mA}$. Em algumas situações, era também possível observar discreto movimento articular de extensão de joelho (quadríceps) ou dorsiflexão de tornozelo (tibial anterior). Em função da acomodação nervosa e da resposta motora, a intensidade era aumentada ao longo das sessões (incremento de $2 \mathrm{~mA}$ a cada 5 minutos, dependendo da visualização da contração muscular e/ou da sensação do paciente).

Figura 2: Paciente recebendo tratamento com EENM nos músculos quadríceps e tibial anterior de acordo com o protocolo do estudo. Nota-se o posicionamento dos eletrodos nas coxas e pernas, e a utilização de fitas adesivas para fixação.

Fonte: autoria própria (2021).

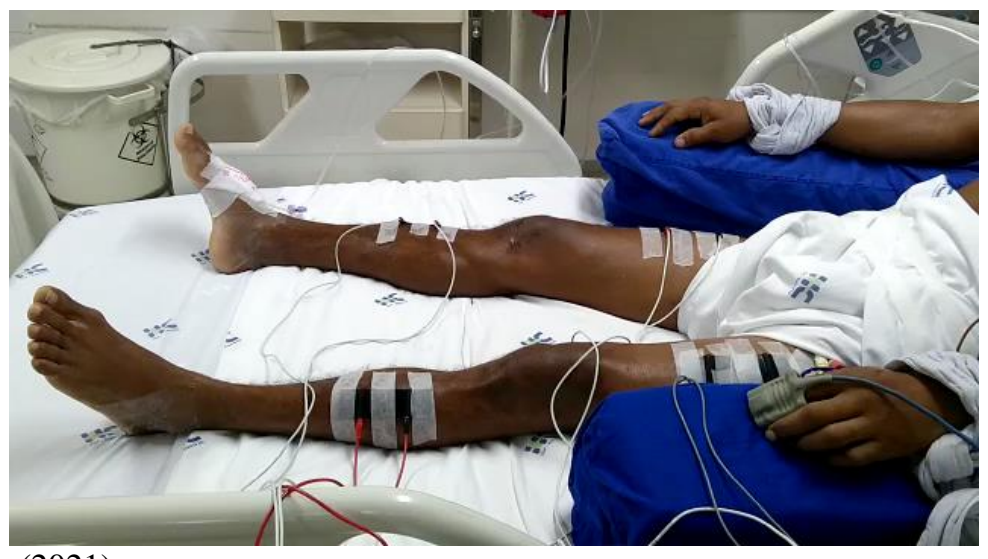




\subsection{Critérios de interrupção da aplicação dos protocolos}

Antes e durante a aplicação dos protocolos de MP e EENM, os fisioterapeutas checavam os sinais vitais e, caso houvesse sinais de instabilidade, a terapia não era iniciada ou era interrompida, com exceção da mobilização passiva ou ativo-assistida leve, desde que houvesse consentimento da equipe médica. Os critérios para interrupção dos protocolos foram:

- Instabilidade cardiovascular: arritmias instáveis ou graves, frequência cardíaca $<60 \mathrm{bpm}$ ou $\geq 130 \mathrm{bpm}$

- Temperatura corporal menor que $36^{\circ} \mathrm{C}$ ou maior que $38^{\circ} \mathrm{C}$;

- Saturação arterial de oxigênio < 90\% persistente ao levantar-se ou deitar-se (a menos que seja a saturação basal do paciente);

- Pressão arterial média menor que $60 \mathrm{mmHg}$ ou maior que $110 \mathrm{mmHg}$;

- Doses crescentes de vasopressores nas últimas 2 horas;

- Paciente agitado impossibilitando terapia;

- Dor incontrolável;

- Cuidados paliativos instituídos ao longo da internação;

- Sinais de trombose venosa profunda (não era realizado no membro afetado).

\subsection{Coleta de dados}

Na tabela 4, encontra-se um resumo das avaliações realizadas. No momento da admissão na UTI, dados demográficos e características clínicas dos pacientes foram obtidas, incluindo a aplicação de escores e escalas que determinam a gravidade da doença, prognóstico e disfunção orgânica, como Índice de comorbidade de Charlson, Simplified Acute Physiology Score 3 (SAPS 3) e o Sequential Organ Failure Assessment (SOFA).

Também foram coletados dados referentes ao tempo de internação, medicamentos utilizados, número de dias de intubação, traqueostomia e VM, antecedentes pessoais, comorbidades e efeitos adversos como: quedas, extubação acidental, angina, arritmias e convulsões durante a aplicação dos protocolos. A incidência de delirium foi avaliada pela escala CAM-ICU ${ }^{(81,82)}$ todos os dias de internação até a alta da UTI, pela equipe de enfermagem durante o período da manhã. 
Para análise do status funcional, foi aplicada a Escala de Estado Funcional em Unidade de Terapia Intensiva ou, em inglês, Functional Status Score for the Intensive Care Unit (FSS-ICU) ${ }^{(49,83)}$ (ANEXO A) e o Teste de Função Física na unidade de terapia intensiva $(\text { PFIT })^{(84)}$ (ANEXO B). Estas escalas foram aplicadas no primeiro dia em que o paciente despertava e apresentava nível de consciência adequado para responder a pelo menos três das seguintes ordens (“abrir/fechar os olhos", "olhar para mim”, "coloque a língua para fora”, "balance sua cabeça", "levante as sobrancelhas") ${ }^{(85)}$, e posteriormente, na alta da UTI e na alta hospitalar.

A escala FSS-ICU é dividida em 5 itens/tarefas: rolar, transferir da posição supina para sentada, transferir da posição sentada para a posição de pé, sentar na beira da cama, e andar. Cada um destes itens recebe uma pontuação da 1 a 7 , em que 1 significa menor independência e 7 significa maior independência, variando de 7 a 35 pontos possíveis. Já a escala PFIT é dividida 4 itens: assistência (sentar para levantar), cadência (passos/minuto), ombro (força de flexão) e joelho (força de extensão). Cada item pode ser pontuado de 0 a 3 , em que 0 significa menor independência e 3 significa maior independência, variando de 0 a 12 pontos possíveis.

Para avaliação da força muscular global, foi utilizada a escala Medical Research Council (MRC) ${ }^{(86,87)}$ (ANEXO C), que pontua de 0 a 5 a força muscular de flexores de ombro, flexores de cotovelo, extensores de punho, flexores de quadril, extensores de joelho e dorsiflexores de tornozelo. Esta escala também foi aplicada no primeiro dia acordado, na alta da UTI e na alta hospitalar. O paciente foi classificado com ICU-AW quando apresentou MRC $<48$ pontos na alta da $\mathrm{UTI}^{(88)}$.

O índice de Barthel $^{(89)}$ (ANEXO D) foi aplicado de forma retrógrada com os familiares do paciente na admissão da UTI para se obter uma estimativa do grau de independência funcional basal pré-internação. Posteriormente, foi aplicado na alta da UTI e na alta hospitalar. Esta escala possui 10 itens: alimentação, banho, atividades rotineiras, vestir-se, função intestinal, sistema urinário, uso do banheiro, transferência, mobilidade e escadas. A pontuação vai de 0 a 100, em que 0 significa menor independência e 100 significa maior independência.

Para análise da qualidade de vida, foi aplicado o questionário Euro Quality of life5 dimensions (EQ-5D) ${ }^{(90,91)}$ (ANEXO E) no momento da alta da UTI e na alta hospitalar. O EQ-5D avalia 5 domínios (mobilidade, cuidados pessoas, atividades habituais, dor/mal estar e ansiedade/depressão em uma escala de 3 pontos (em que "1" demonstra mais independência e/ou a não apresentação de problemas em determinado item e "3" demonstra menor 
independência e/ou presença de problemas), além de avaliar o estado de saúde, em que o próprio paciente determina uma pontuação de 0 a 100 de acordo com uma escala visual analógica. Para análise das fibras musculares, foi realizado exame de ultrassom ${ }^{(25,92)}$ por dois médicos treinados, na admissão e na alta da UTI, no músculo quadríceps.

Tabela 4: Avaliações e escalas utilizadas e os respectivos momentos de avaliação.

\begin{tabular}{c|c|c|c|c|c|c|c}
\hline Avaliação & $\begin{array}{l}\text { Charlson } \\
\text { SAPS 3 } \\
\text { SOFA }\end{array}$ & $\begin{array}{c}\text { CAM- } \\
\text { ICU }\end{array}$ & MRC & US & $\begin{array}{l}\text { FSS-ICU } \\
\text { PFIT }\end{array}$ & $\begin{array}{c}\text { Índice de } \\
\text { Barthel }\end{array}$ & EQ-5D \\
\hline Admissão na UTI & $\mathrm{X}$ & & & $\mathrm{X}$ & & $\mathrm{X} *$ & \\
\hline Primeiro dia consciente & & & $\mathrm{X}$ & & $\mathrm{X}$ & & \\
\hline Todos os dias na UTI & & $\mathrm{X}$ & & & & & $\mathrm{X}$ \\
\hline Alta da UTI & & & $\mathrm{X}$ & $\mathrm{X}$ & $\mathrm{X}$ & $\mathrm{X}$ & $\mathrm{X}$ \\
\hline Alta Hospitalar & & & $\mathrm{X}$ & & $\mathrm{X}$ & $\mathrm{X}$ & $\mathrm{X}$ \\
\hline
\end{tabular}

SAPS 3: Simplified Acute Physiology Score; SOFA: Sequential Organ Failure Assessment; CAM-

ICU: Confusion Assessment Method in Intensive Care Unit; MRC: Medical Research Council; FSS-

ICU: Escala de Estado Funcional em Unidade de Terapia Intensiva; PFIT: Teste de Função Física na unidade de terapia intensiva; US: Ultrassom de quadríceps; EQ-5D: Euro Quality of life-5 dimensions. *Índice de Barthel pré-internação, aplicado com a família do paciente na admissão da UTI.

\subsection{Análise estatística}

A amostra do estudo foi calculada baseando-se nos dados obtidos em nosso hospital e em estudos prévios ${ }^{(57,93)}$. Considerando uma alteração mínima de 7 pontos na escala FSS-ICU, assumindo um nível de significância de 5\%, um poder de 80\% (beta = 20\%) e um desvio padrão de 8 , são necessários 21 pacientes em cada grupo no desfecho primário FSS-ICU na alta da UTI.

Para testar a distribuição da amostra, foram utilizados os testes de normalidade Shapiro-Wilk e D'Agostino \& Pearson. As variáveis categóricas foram expressas em porcentagem. As variáveis contínuas foram expressas em média e desvio padrão quando apresentaram distribuição normal, e mediana e intervalo interquartil quando apresentaram distribuição não-normal. Para as variáveis categóricas, foi utilizado o teste de Fisher para análise entre dois grupos e o teste do Qui-Quadrado para vários grupos. Para variáveis contínuas com distribuição normal, foi utilizado o teste $\mathrm{t}$ student. Para variáveis com 
distribuição não normal, foi utilizado o teste não paramétrico de Mann Whitney. Foi considerado significativo um valor de $\mathrm{p}<0,05$. As análises foram realizadas no programa GraphPadPrism ${ }^{\circledR}$ e SPSS ${ }^{\circ}$.

\subsection{Aspectos éticos}

Em observância a legislação que regulamenta a pesquisa com seres humanos, o protocolo de pesquisa foi submetido e iniciado somente após aprovação do Comitê de Ética em Pesquisa do HCFMRP-USP (CAAE: 72872117.2.0000.5440). O estudo foi publicado no Registro Brasileiro de Ensaios Clínicos (ReBEC: https://ensaiosclinicos.gov.br; RBR$3 \mathrm{w} 3 \mathrm{kmg})$.

O Termo de Consentimento Livre e Esclarecido tinha uma linguagem simples. Os participantes ou os seus representantes legais foram convidados a participar da pesquisa e informados sobre os objetivos, procedimentos, riscos, desconfortos, benefícios, garantia do anonimato e que a participação deles era voluntária. Daqueles que concordaram em participar, foi solicitado anuência mediante assinatura do TCLE. O estudo foi conduzido em conformidade com as resoluções nacionais e internacionais como descritas nos seguintes documentos: Resolução CNS 466/12, Declaração de Helsinque e todas as suas revisões e alterações e o Documento de Las Américas. 


\section{Resultados}

\subsection{Caracterização da amostra}

Neste ensaio clínico, 451 pacientes foram triados e 139 foram incluídos. Deste total, 69 pacientes foram randomizados para o grupo MP e 70 no grupo MP+EENM. Quarenta pacientes completaram o protocolo no grupo MP e 34 pacientes completaram o protocolo no grupo MP+EENM (Figura 3). A coleta de dados foi realizada até atingir uma amostra de pelo menos 21 pacientes na escala FSS-ICU em ambos os grupos na alta da UTI.

Figura 3: Fluxograma do estudo.

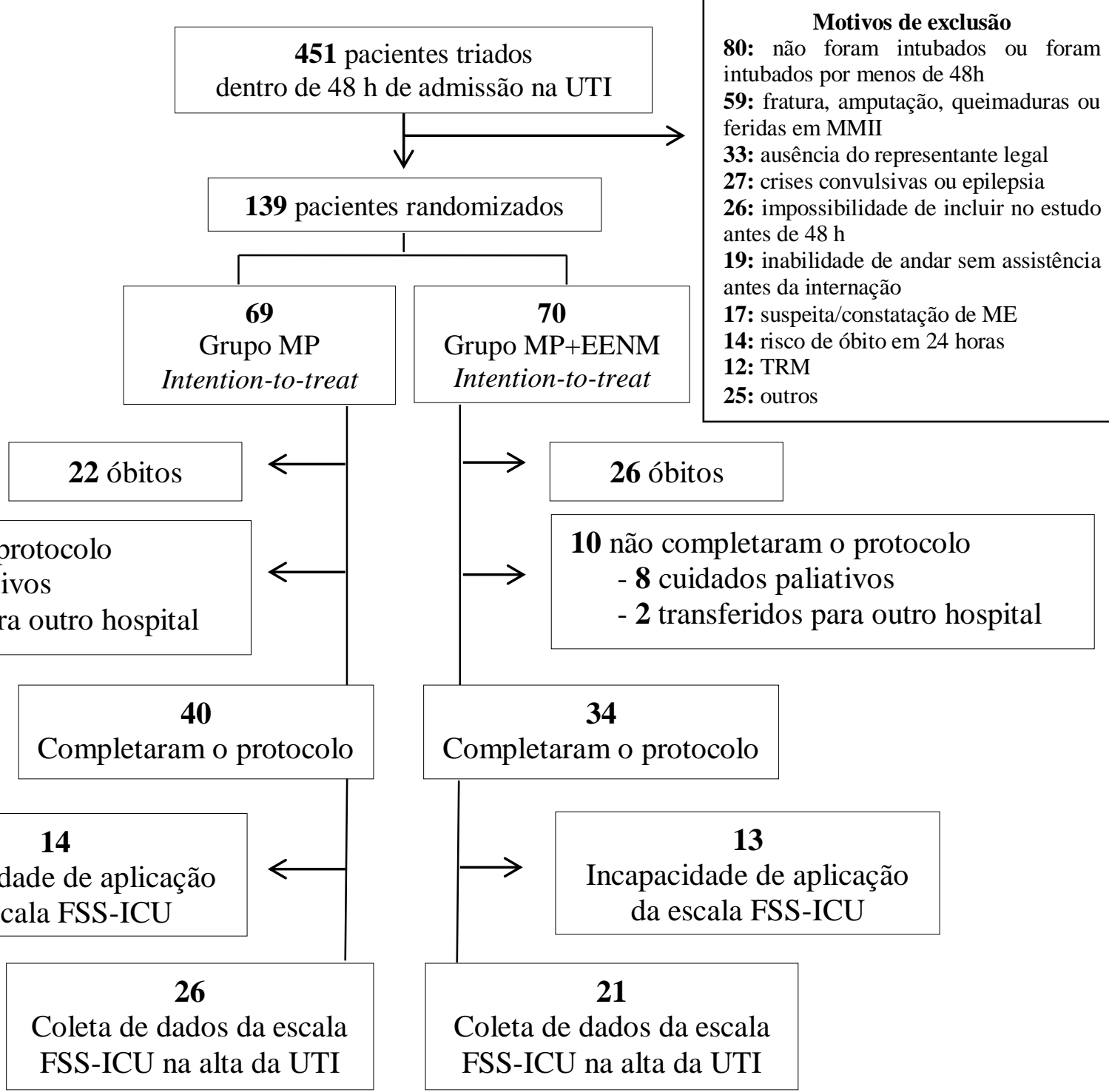

MP: Mobilização Precoce; EENM: Estimulação elétrica neuromuscular; FSS-ICU: Escala de Estado Funcional em Unidade de Terapia Intensiva; MMII: membros inferiores; TCLE: Termo de Consentimento Livre e Esclarecido; h: horas; ME: morte encefálica; TRM: Trauma raqui-medular.

Fonte: autoria própria (2021). 
As características dos pacientes dos dois grupos estão na Tabela 5. Não houve diferenças estatisticamente significantes entre os grupos nas variáveis analisadas (idade, sexo, SAPS 3, SOFA, índice de Charlson, índice de massa corpórea, índice de Barthel, diagnósticos da internação e antecedentes pessoais).

Tabela 5: Caracterização da amostra quanto a idade, sexo, SAPS, SOFA, índice de Charlson, índice de Barthel retrógrado e diagnósticos da internação.

\begin{tabular}{|c|c|c|c|}
\hline Variáveis & $\begin{array}{c}\text { Grupo MP } \\
n=69\end{array}$ & $\begin{array}{c}\text { Grupo MP + EENM } \\
n=70\end{array}$ & $\mathrm{p}$ \\
\hline Idade & $49,68 \pm 17,66$ & $49,57 \pm 17,85$ & 0,971 \\
\hline Sexo Feminino & $42,03 \%$ & $28,57 \%$ & \multirow{2}{*}{0,112} \\
\hline Sexo Masculino & $57,97 \%$ & $71,43 \%$ & \\
\hline SAPS 3 & $69,59 \pm 16,50$ & $69,36 \pm 12,78$ & 0,924 \\
\hline SOFA & $10,04 \pm 2,74$ & $9,81 \pm 3,04$ & 0,642 \\
\hline Índice de Charlson & $2,81 \pm 2,42$ & $2,72 \pm 2,40$ & 0,799 \\
\hline IMC & $26,78 \pm 6,51$ & $26,63 \pm 6,28$ & 0,926 \\
\hline Índice de Barthel retrógrado & $99,41 \pm 2,03$ & $99,57 \pm 1,65$ & 0,694 \\
\hline \multicolumn{4}{|l|}{ Diagnóstico } \\
\hline AVE & $15(21 \%)$ & $22(31 \%)$ & \\
\hline TCE & $18(26 \%)$ & $19(27 \%)$ & \\
\hline Pós-cirúrgico & $43(62 \%)$ & $37(52 \%)$ & \\
\hline Insuficiência respiratória & $36(52 \%)$ & $35(50 \%)$ & 0,827 \\
\hline Sepse & $34(49 \%)$ & $30(43 \%)$ & \\
\hline Outros diagnósticos & $4(6 \%)$ & $3(4 \%)$ & \\
\hline \multicolumn{4}{|l|}{ Antecedentes pessoais } \\
\hline Hipertensão Arterial & $19(27 \%)$ & $25(36 \%)$ & \\
\hline Diabetes & $14(20 \%)$ & $12(17 \%)$ & \\
\hline Tabagismo & $15(22 \%)$ & $21(30 \%)$ & 0,894 \\
\hline Etilismo & $18(26 \%)$ & $17(24 \%)$ & \\
\hline Drogadição & $12(17 \%)$ & $11(16 \%)$ & \\
\hline DPOC & $5(7 \%)$ & $6(9 \%)$ & \\
\hline Previamente hígido & $11(16 \%)$ & $13(19 \%)$ & \\
\hline
\end{tabular}

MP: Mobilização precoce; EENM: Estimulação elétrica neuromuscular; SAPS 3: Simplified Acute Physiology Score 3; SOFA: Sequential Organ Failure Assessment; AVE: Acidente vascular cerebral; TCE: Trauma crânio encefálico; DPOC: Doença pulmonar obstrutiva crônica. 
A Tabela 6 mostra os dias de utilização dos principais medicamentos, tais como sedativos, bloqueadores neuromusculares, drogas vasoativas e antibióticos, durante a internação na UTI, nos dois grupos. Não foram encontradas diferenças significativas entre os grupos.

Tabela 6: Principais medicamentos utilizados durante a internação na UTI.

\begin{tabular}{lccc}
\hline Variáveis & MP & MP + EENM & $\mathrm{p}$ \\
\hline Dias com sedativos & $6,48 \pm 3,92$ & $6,36 \pm 4,37$ & 0,683 \\
Dias com bloqueadores neuromusculares & $1,70 \pm 3,15$ & $1,22 \pm 2,71$ & 0,241 \\
Dias com opioides & $14,76 \pm 9,95$ & $12,24 \pm 7,69$ & 0,141 \\
Dias com dexmetomidina & $0,59 \pm 1,40$ & $0,84 \pm 1,68$ & 0,530 \\
Dias com benzodiazepínicos & $3,42 \pm 5,21$ & $4,00 \pm 4,68$ & 0,291 \\
Dias com drogas vasoativas & $7,09 \pm 4,93$ & $5,26 \pm 4,28$ & 0,054 \\
Dias com antibióticos & $13,09 \pm 8,55$ & $10,38 \pm 6,47$ & 0,058 \\
\hline
\end{tabular}

MP: Mobilização precoce; EENM: estimulação elétrica neuromuscular.

As variáveis estão expressas em média \pm desvio padrão.

Fonte: autoria própria (2021).

\subsection{Características dos protocolos de MP e EENM}

Não houve efeitos adversos relacionados à aplicação dos protocolos. O tempo para se iniciar o protocolo de MP foi semelhante nos dois grupos (15h48min $\pm 8 \mathrm{~h} 42 \mathrm{~min}$ no grupo MP vs. $15 \mathrm{~h} 44 \mathrm{~min} \pm 12 \mathrm{~h} 26 \mathrm{~min}$ no grupo MP+EENM). Em relação ao protocolo de EENM, ele foi iniciado em 33h52min \pm 12h30min após a admissão na UTI, com uma média de 7,22 \pm 3,70 sessões por paciente, totalizando 422 sessões de EENM (Tabela 7).

Tabela 7: Avaliação da aplicação do protocolo de MP e de EENM.

\begin{tabular}{lccc}
\hline Variáveis & MP & MP+EENM & $\mathrm{p}$ \\
\hline Tempo para início da MP & 15h48min $\pm 8 \mathrm{~h} 42 \mathrm{~min}$ & $15 \mathrm{~h} 44 \mathrm{~min} \pm 12 \mathrm{~h} 26 \mathrm{~min}$ & 0,334 \\
Número de sessões de fisioterapia motora & $17,90 \pm 13,47$ & $15,24 \pm 11,25$ & 0,281 \\
Tempo para início da EENM & ----- & $33 \mathrm{~h} 52 \mathrm{~min} \pm 12 \mathrm{~h} 30 \mathrm{~min}$ & ------ \\
Número de sessões de EENM realizadas & ------ & $7,22 \pm 3,70$ & ------ \\
\hline
\end{tabular}

MP: Mobilização precoce; EENM: estimulação elétrica neuromuscular; h: hora; min: minuto.

As variáveis estão expressas em média \pm desvio padrão.

Fonte: autoria própria (2021). 
Os valores de frequência cardíaca (FC), pressão arterial média (PAM), frequência respiratória (FR) e saturação arterial de oxigênio $\left(\mathrm{SpO}_{2}\right)$ coletados antes e após as sessões de EENM encontram-se na tabela 8. Houve diferenças estatísticas apenas em relação a $\mathrm{SpO}_{2}$, em que a média foi de 96,30 \pm 2,61 antes da EENM para 96,6 \pm 2,47 após a EENM, com $\mathrm{p}=0,001$.

Tabela 8: Valores de FC, PAM, FR e $\mathrm{SpO}_{2}$ antes e depois das sessões de EENM e a média da diferença entre os dois momentos

\begin{tabular}{lccc}
\hline Variáveis & Antes da EENM & Após a EENM & $\mathrm{p}$ \\
\hline FC & $99,60 \pm 19,22$ & $99,70 \pm 19,52$ & 0,739 \\
PAM & $88,39 \pm 14,60$ & $88,71 \pm 14,76$ & 0,398 \\
FR & $22,08 \pm 7,35$ & $22,09 \pm 7,27$ & 0,880 \\
$\mathrm{SpO}_{2}$ & $96,30 \pm 2,61$ & $96,6 \pm 2,47$ & 0,001
\end{tabular}

EENM: Estimulação elétrica neuromuscular; FC: Frequência cardíaca; PAM: Pressão arterial média; FR: Frequência respiratória e $\mathrm{SpO}$ 2: Saturação arterial de oxigênio.

Fonte: autoria própria (2021).

\subsection{Tamanho da amostra de acordo com o desfecho analisado}

Devido às condições clínicas dos pacientes, nem todas as avaliações puderam ser realizadas em todos os momentos. Assim, na tabela 9 encontra-se o número de pacientes que realizou cada avaliação. 
Tabela 9: Número de pacientes em cada avaliação nos diferentes momentos.

\begin{tabular}{|c|c|c|c|}
\hline & Grupo MP & Grupo MP+EENM & Total \\
\hline \multicolumn{4}{|l|}{ Pré-internação } \\
\hline Índice de Barthel, n & 38 & 34 & 137 \\
\hline \multicolumn{4}{|l|}{$1^{\circ}$ dia acordado } \\
\hline FSS-ICU, n & 23 & 20 & 43 \\
\hline PFIT, $\mathrm{n}$ & 22 & 18 & 40 \\
\hline $\mathrm{MRC}, \mathrm{n}$ & 18 & 15 & 33 \\
\hline \multicolumn{4}{|l|}{ Alta da UTI } \\
\hline FSS-ICU, n & 26 & 21 & 47 \\
\hline PFIT, $\mathrm{n}$ & 22 & 18 & 40 \\
\hline MRC, $\mathrm{n}$ & 22 & 18 & 40 \\
\hline EQ-5D, n & 25 & 18 & 43 \\
\hline Índice de Barthel, n & 38 & 34 & 72 \\
\hline \multicolumn{4}{|l|}{ Alta Hospitalar } \\
\hline FSS-ICU, n & 26 & 24 & 50 \\
\hline PFIT, $\mathrm{n}$ & 26 & 21 & 47 \\
\hline $\mathrm{MRC}, \mathrm{n}$ & 23 & 22 & 45 \\
\hline EQ-5D, n & 26 & 21 & 47 \\
\hline Índice de Barthel, $\mathrm{n}$ & 36 & 33 & 69 \\
\hline
\end{tabular}

MP: Mobilização precoce; EENM: Estimulação elétrica neuromuscular; Barthel: Índice de Barthel; FSS-ICU: Functional Status Score for the Intensive Care Unit; PFIT: Teste de Função Física na unidade de terapia intensiva; MRC: Medical Research Council; EQ-5D: Euro Quality of life-5 dimension, UTI: Unidade de Terapia intensiva.

Fonte: autoria própria (2021). 


\subsection{Desfechos Funcionais}

\subsubsection{Escala FSS-ICU}

Na Figura 4 encontram-se os resultados da escala FSS-ICU aplicada em três momentos: $1^{\mathrm{o}}$ dia consciente, alta da UTI e alta hospitalar. Os pacientes do grupo MP+EENM apresentaram valores significativamente maiores do que os pacientes do grupo MP em todos os momentos avaliados. Primeiro dia acordado: $15.2 \pm 8.4$ no grupo MP vs. $21.2 \pm 6.8$ no grupo MP+EENM (p=0.0154). Alta da UTI: $18.9 \pm 8.6$ no grupo MP vs. $26.3 \pm 7.8$ no grupo MP+EENM (p=0.0039). Alta hospitalar: $24.5 \pm 8.7$ vs. $29 \pm 8.8(\mathrm{p}=0.0144)$, respectivamente.

Figura 4: Avaliação do estado funcional, utilizando-se a escala FSS-ICU, nos grupos MP e MP+EENM em diferentes momentos da internação.
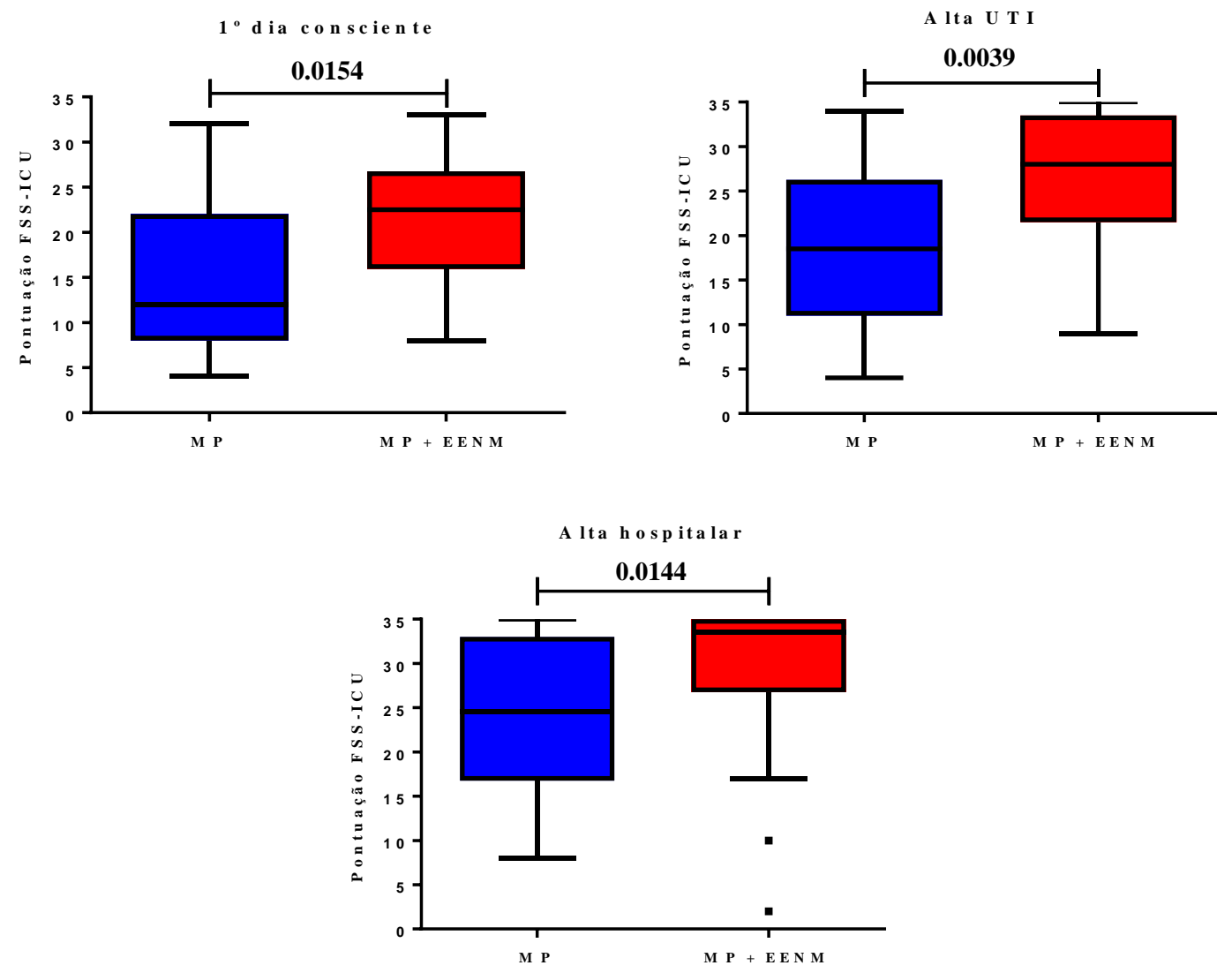

MP: Mobilização precoce; EENM: Estimulação elétrica neuromuscular; UTI: Unidade de terapia intensiva; FSS-ICU: Functional Status Score for the Intensive Care Unit.

Fonte: autoria própria (2021). 


\subsubsection{Escala PFIT}

Quando o status funcional foi avaliado com a escala PFIT, os pacientes do grupo MP+EENM também apresentaram valores significativamente maiores do que os pacientes do grupo MP em todos os momentos avaliados (Figura 5). No $1^{\circ}$ dia consciente na UTI os valores são $8.18 \pm 2.45$ vs $5.80 \pm 2.88$ ( $\mathrm{p}=0,0189)$, na alta da UTI os valores são $10.28 \pm 2.34$ vs $6.90 \pm 2.56(\mathrm{p}=0,0001)$ e na alta hospitalar os valores são $10.52 \pm 2.27$ vs $8.61 \pm 2.65$ $(\mathrm{p}=0,0054)$, respectivamente.

Figura 5: Avaliação do estado funcional, utilizando-se a escala PFIT, nos grupos MP e MP+EENM em diferentes momentos da internação.
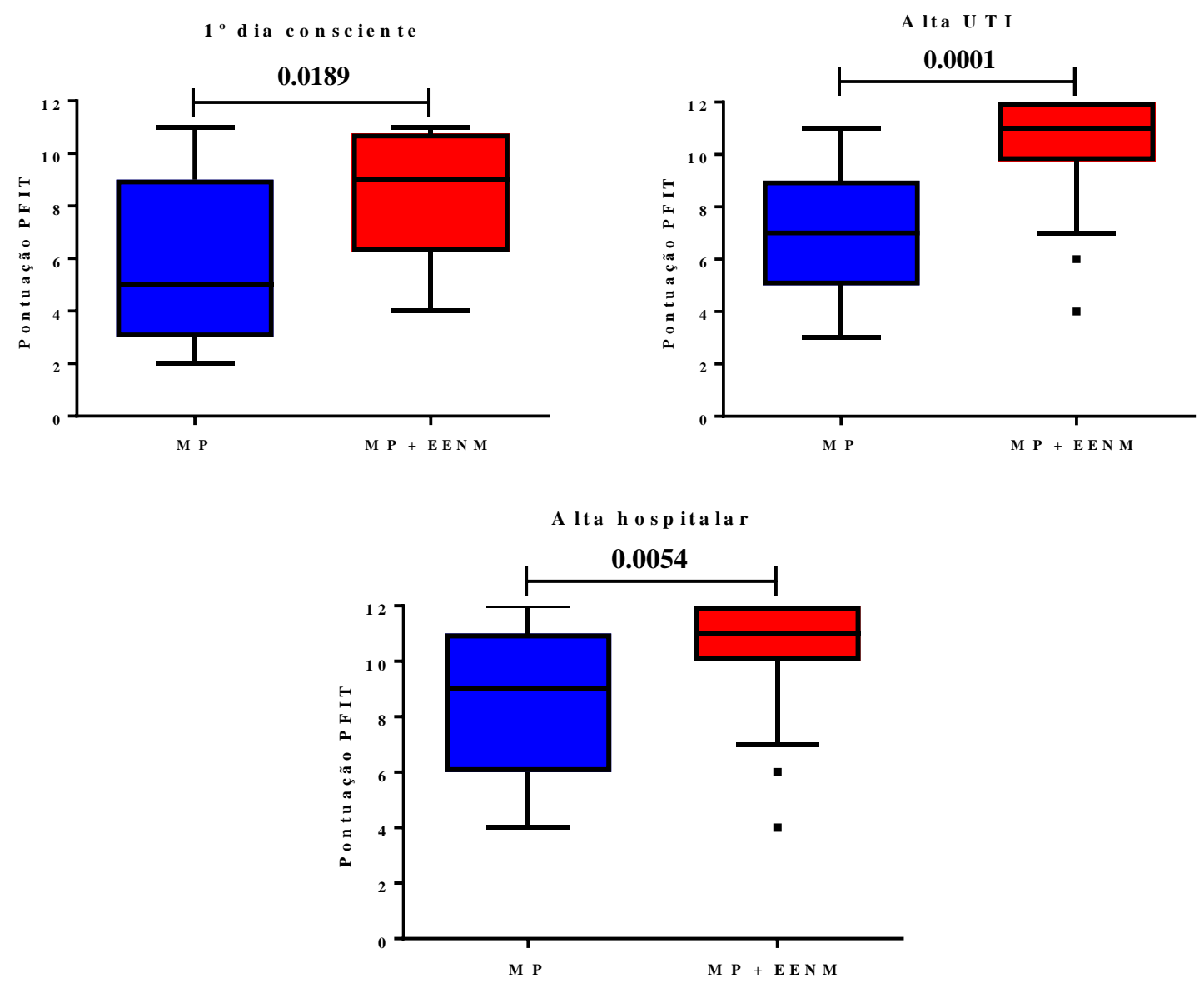

MP: Mobilização precoce; EENM: Estimulação elétrica neuromuscular; UTI: Unidade de terapia intensiva; PFIT: Teste de Função Física na unidade de terapia intensiva.

Fonte: autoria própria (2021). 


\subsubsection{Avaliação da força muscular e incidência de ICU-AW}

Os pacientes do grupo MP+EENM apresentaram valores significativamente maiores de força muscular do que os pacientes do grupo MP nos três momentos avaliados. Os valores de MRC total e de MRC de membros inferiores (MMII) estão dispostos na tabela 10. A figura 6 demonstra a representação gráfica dos valores de MRC nos três momentos de avaliação. O grupo MP+EENM apresentou incidência significativamente menor de ICU-AW do que o grupo MP (11\% vs. $45 \%$; $\mathrm{p}=0,035)$.

Tabela 10: Valores de MRC total e de MMII no primeiro dia consciente, na alta da UTI e na alta hospitalar.

\begin{tabular}{lccc}
\hline Desfecho & MP & MP+EENM & $p$ \\
\hline Primeiro dia consciente & $42(33-54)$ & $54(47-60)$ & 0,0111 \\
MRC total & $22(18-27)$ & $30(24-30)$ & 0,0056 \\
MRC MMII & & & \\
Alta da UTI & $50(39-56)$ & $58,5(55-60)$ & 0,0007 \\
MRC total & $26,5(20-28)$ & $30(28-30)$ & 0,0003 \\
MRC MMII & & $59(56-60)$ & 0,0105 \\
Alta Hospitalar & $52(49-59)$ & $30(29-30)$ & 0,0103 \\
MRC total & $28(23-30)$ & & \\
MRC MMII &
\end{tabular}

MRC: Medical Research Council; MMII: membros inferiores; UTI: unidade de terapia intensiva.

Fonte: autoria própria (2021). 
Figura 6: Avaliação da força muscular global nos grupos MP e MP+EENM em diferentes momentos da internação.
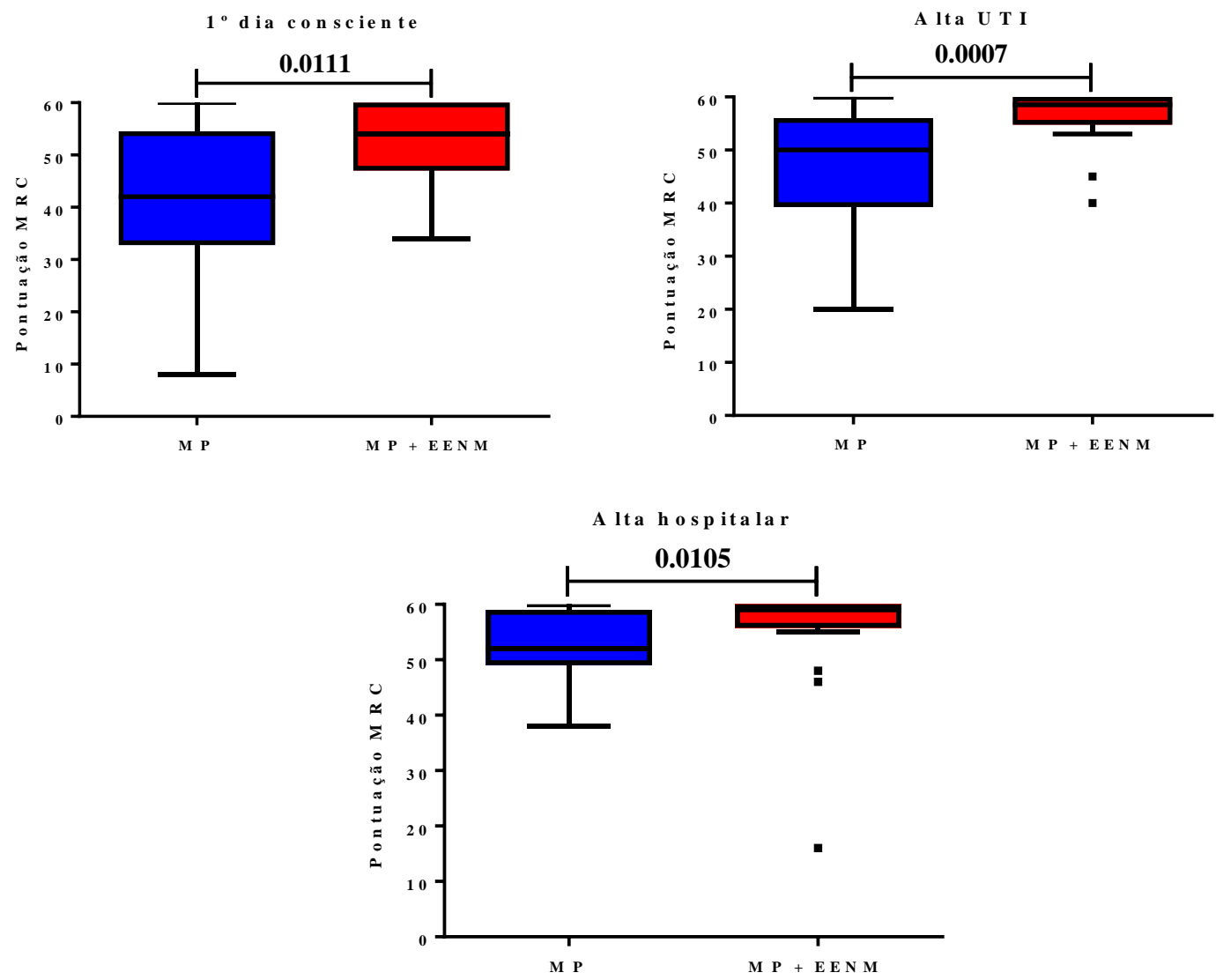

MP: Mobilização precoce; EENM: Estimulação elétrica neuromuscular; UTI: Unidade de terapia intensiva; MRC: Medical Research Council.

Fonte: autoria própria (2021). 


\subsubsection{Avalição do estado de independência funcional}

A Figura 7 mostra os resultados referentes ao estado de independência funcional, avaliada pelo o Índice de Barthel, na alta da UTI e na alta hospitalar. Não houve diferenças estatísticas entre os grupos.

Figura 7: Avaliação do estado de independência funcional nos grupos MP e MP+EENM em diferentes momentos da internação.
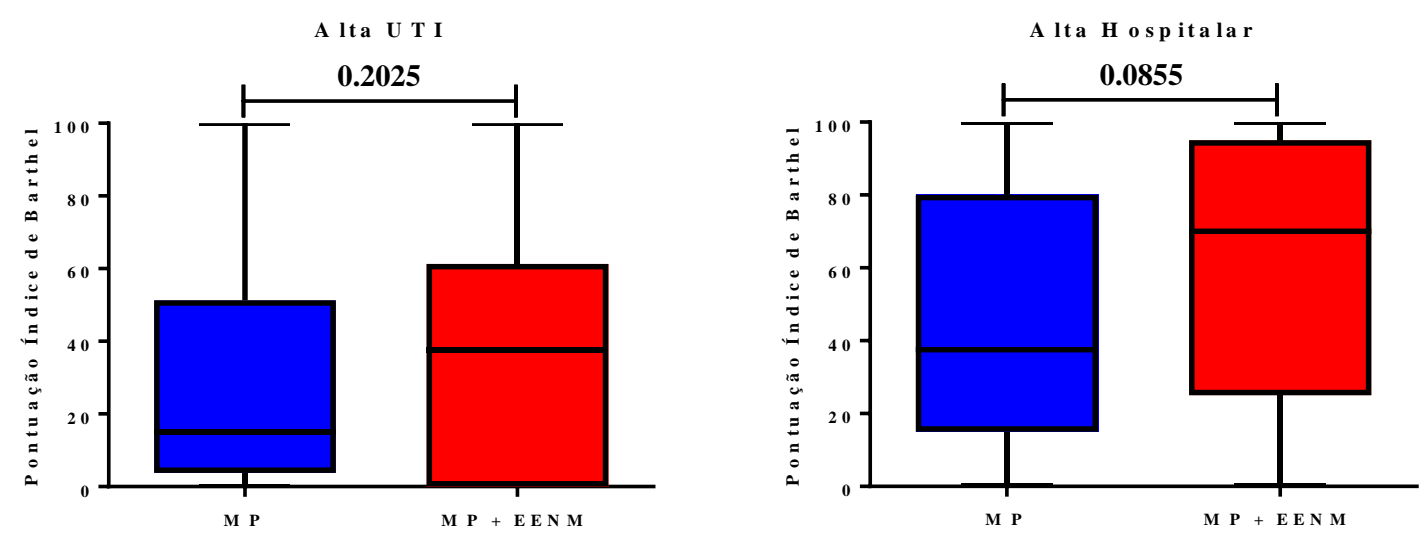

MP: Mobilização precoce; EENM: Estimulação elétrica neuromuscular; UTI: Unidade de terapia intensiva.

Fonte: autoria própria (2021). 


\subsection{Análise dos marcos motores}

Os grupos receberam uma quantidade semelhante de sessões de fisioterapia motora: 17 (8-26) no grupo MP vs. 15 (10-22) no grupo MP+EENM. Não houve diferenças significativas no número de pacientes que atingiram cada marco motor nos grupos estudados (Tabela 11).

Tabela 11: Número de pacientes sobreviventes que realizaram cada marco motor.

\begin{tabular}{lccc}
\hline Marco motor & $\begin{array}{c}\text { Grupo MP } \\
\mathrm{n}=47\end{array}$ & $\begin{array}{c}\text { Grupo MP+EENM } \\
\mathrm{n}=44\end{array}$ & $\mathrm{p}$ \\
\hline Sentar na beira do leito, $\mathrm{n}$ & $34(72 \%)$ & $29(65 \%)$ & \\
Sentar na poltrona, $\mathrm{n}$ & $34(72 \%)$ & $29(65 \%)$ & \\
Ortostatismo, $\mathrm{n}$ & $29(61 \%)$ & $25(56 \%)$ & 0,916 \\
Marcha estacionária, $\mathrm{n}$ & $11(23 \%)$ & $11(25 \%)$ & \\
Deambular, $\mathrm{n}$ & $16(34 \%)$ & $19(43 \%)$ & \\
\hline
\end{tabular}

MP: mobilização precoce; EENM: Eletroestimulação neuromuscular. Fonte: autoria própria (2021).

Na figura 8 encontra-se o número de dias até a primeira vez em que as seguintes atividades foram realizadas: sentar na beira do leito, sentar na poltrona, ortostatismo e deambular. Os pacientes do grupo MP+EENM conseguiram ficar em pé significativamente antes do que os pacientes do grupo MP: 8 (5-11) dias vs. 10 (6-13) dias ( $\mathrm{p}=0.036)$, respectivamente. Nos demais marcos, não houve diferenças significativas entre os grupos. 
Figura 8: Curvas Kaplan-Meier referentes ao número de dias de internação na UTI até a primeira vez em que cada atividade foi realizada.
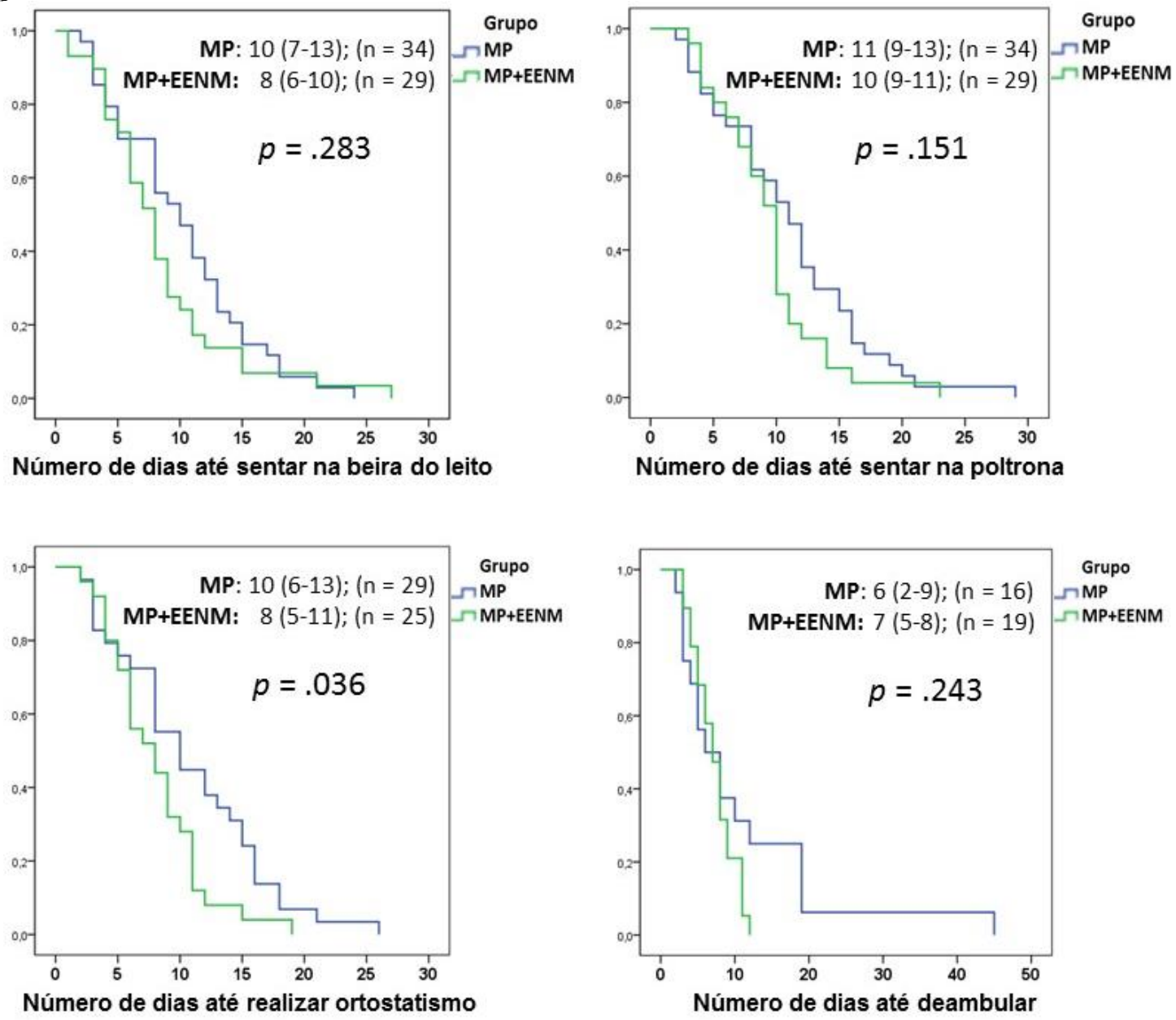

MP: mobilização precoce. EENM: Estimulação elétrica neuromuscular.

As variáveis estão expressas em mediana (intervalo interquartil).

Fonte: autoria própria (2021).

\subsection{Desfechos clínicos}

\subsubsection{Mortalidade e tempo de ventilação mecânica}

Houve 22 óbitos no grupo MP e 26 no grupo MP+EENM ( $\mathrm{p}=0,593)$ até a alta da UTI e um total de 24 óbitos no grupo MP e 30 no grupo MP+EENM até a alta hospitalar ( $\mathrm{p}=0,385$ ). Quanto aos dias de VM, a média foi de 11,94 $\pm 6,09$ no grupo MP e 10,72 $\pm 6,91$ no grupo MP+EENM ( $\mathrm{p}=0,135)$ (Tabela 12). 
Tabela 12: Mortalidade na alta da UTI e na alta hospitalar e dias de ventilação mecânica.

\begin{tabular}{lccc}
\hline Variáveis & Grupo MP & Grupo MP + EENM & P \\
& $\mathrm{n}=69$ & $\mathrm{n}=70$ & \\
\hline Mortalidade na alta da UTI & $22(31,88 \%)$ & $26(37,14 \%)$ & 0,593 \\
Mortalidade na alta hospitalar & $24(34,78 \%)$ & $30(42,86 \%)$ & 0,385 \\
Dias de ventilação mecânica & $11,94 \pm 6,09$ & $10,72 \pm 6,91$ & 0,135
\end{tabular}

MP: Mobilização precoce; EENM: Estimulação elétrica neuromuscular; UTI: Unidade de terapia intensiva.

Fonte: autoria própria (2021).

\subsubsection{Tempo de internação na UTI e hospitalar}

Na figura 9 encontra-se o tempo de internação na UTI e hospitalar. Não houve diferença estatisticamente significante em relação aos dias de internação na UTI entre os grupos $(\mathrm{p}=0,061)$. Por outro lado, os pacientes do grupo MP+EENM apresentaram significativamente menos dias de internação hospitalar do que os pacientes do grupo MP $(24,11 \pm 18,98$ vs. $32,01 \pm 26,25 ; \mathrm{p}=0,048)$.

Figura 9: Tempo de internação na UTI e tempo de internação hospitalar nos grupos MP e MP+EENM.
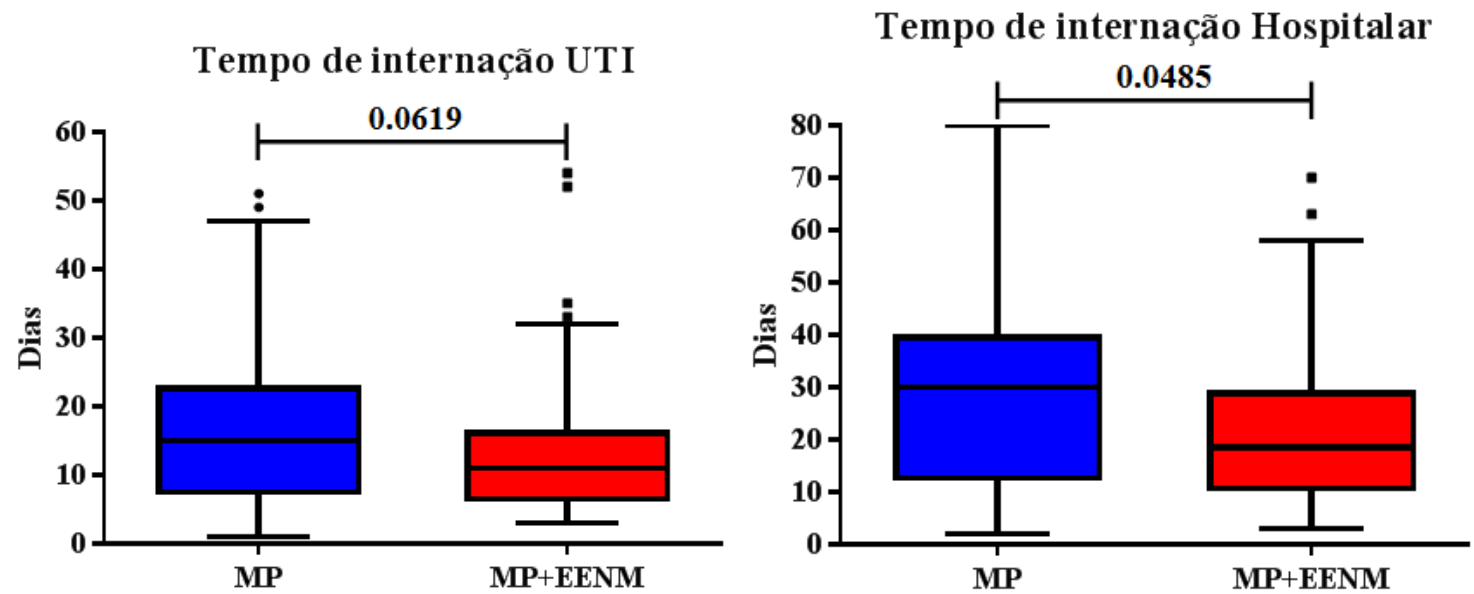

UTI: Unidade de terapia intensiva; MP: Mobilização precoce; EENM: Estimulação elétrica neuromuscular; DP: Desvio padrão.

Fonte: autoria própria (2021). 


\subsubsection{Ultrassom de quadríceps}

Em relação às medidas do quadríceps por ultrassom, foram considerados apenas os dados dos pacientes que tiveram coletas nos dois momentos (admissão e alta da UTI), obtendo-se uma amostra final de 14 pacientes no grupo MP e 10 pacientes no grupo EENM. Não houve diferença significativa na espessura do quadríceps avaliado por ultrassom nos pacientes de um mesmo grupo quando comparada a admissão e a alta da UTI (figura 10) e quando um grupo foi comparado ao outro nos dois momentos (tabela 13).

Figura 10: Medidas de ultrassom de quadríceps na admissão e na alta da UTI no grupo MP e no grupo MP+EENM.

Ultrassom de quadríceps - grupo MP

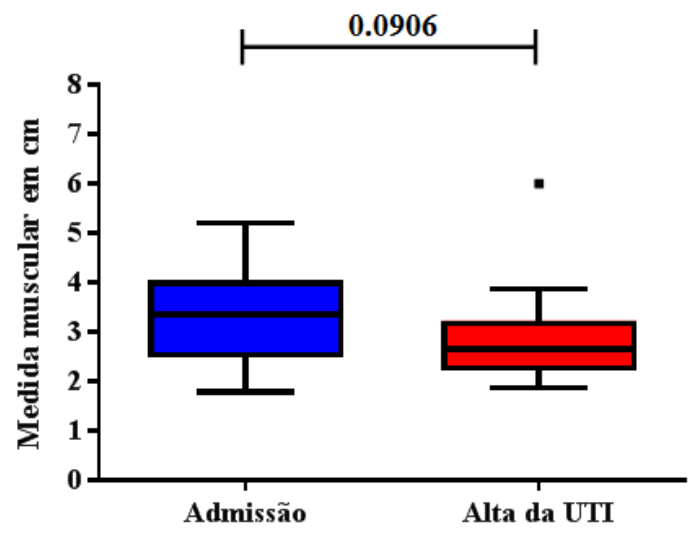

Ultrassom de quadríceps - grupo MP+EENM

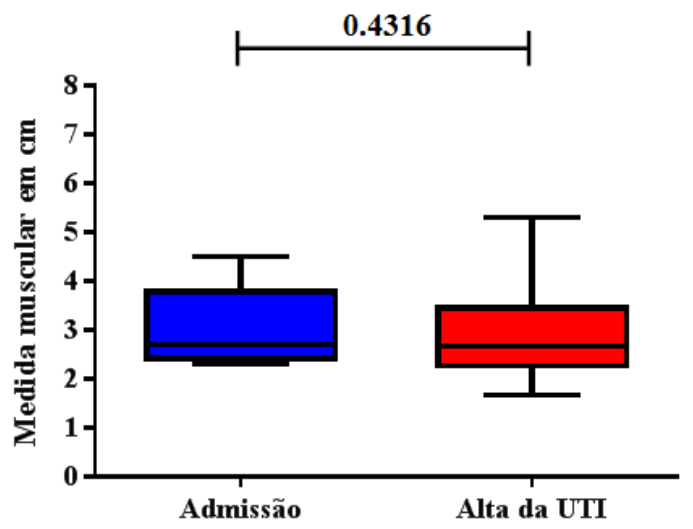

Fonte: autoria própria (2021).

Tabela 13: Medidas da camada muscular de quadríceps, comparando os grupos MP e MP+EENM na admissão da UTI e na alta da UTI: análise não pareada.

\begin{tabular}{lccc}
\hline Medida de quadríceps por ultrassom & Grupo MP & Grupo MP+EENM & P \\
\hline Admissão na UTI (cm) & $n=14$ & $n=10$ & \\
Alta da UTI (cm) & $3,27 \pm 0,97$ & $3,05 \pm 0,81$ & 0,575 \\
Alta da UTI - Admissão na UTI (cm) & $2,90 \pm 1,04$ & $2,93 \pm 1,06$ & 0,873 \\
\hline
\end{tabular}

Fonte: autoria própria (2021). 


\subsection{Avaliação da qualidade de vida e da incidência de delirium}

Os valores da escala de qualidade de vida, avaliados pelo questionário EQ-5D, estão expressos na tabela 14. A incidência de delirium está expressa na tabela 15 em dois momentos de avaliação (alta da UTI e alta hospitalar). Não houve diferenças estatísticas entre os grupos MP e MP+EENM em nenhum dos momentos de avaliação em relação à melhora na qualidade de vida e à incidência de delirium.

Tabela 14: Avaliação da qualidade de vida na alta da UTI e na alta hospitalar

\begin{tabular}{|c|c|c|c|c|c|}
\hline \multicolumn{3}{|c|}{ ALTA DA UTI } & \multicolumn{3}{|c|}{ ALTA HOSPITALAR } \\
\hline MP & MP+EENM & $\mathrm{p}$ & MP & MP+EENM & $\mathrm{P}$ \\
\hline \multicolumn{6}{|l|}{ Mobilidade* } \\
\hline $2,42 \pm 0,50$ & $2,16 \pm 0,51$ & 0,140 & $1,72 \pm 2,28$ & $1,49 \pm 2,21$ & 0,467 \\
\hline \multicolumn{6}{|c|}{ Cuidados Pessoais* } \\
\hline $2,45 \pm 2,58$ & $2,03 \pm 2,62$ & 0,902 & $1,90 \pm 2,56$ & $1,43 \pm 2,18$ & 0,101 \\
\hline \multicolumn{6}{|c|}{ Atividades Habituais* } \\
\hline $2,61 \pm 0,57$ & $2,50 \pm 0,51$ & 0,477 & $2,16 \pm 2,68$ & $1,71 \pm 2,38$ & 0,086 \\
\hline \multicolumn{6}{|c|}{ Dor e mal estar* } \\
\hline $1,60 \pm 2,00$ & $1,38 \pm 2,05$ & 0,541 & $1,37 \pm 1,93$ & $1,36 \pm 1,96$ & 0,938 \\
\hline \multicolumn{6}{|c|}{ Ansiedade e Depressão* } \\
\hline $1,86 \pm 2,51$ & $1,77 \pm 2,44$ & 0,693 & $2,01 \pm 2,60$ & $1,71 \pm 2,38$ & 0,224 \\
\hline \multicolumn{3}{|c|}{ ALTA DA UTI } & \multicolumn{3}{|c|}{ ALTA HOSPITALAR } \\
\hline MP & MP+EENM & $\mathrm{P}$ & MP & MP+EENM & $\mathrm{P}$ \\
\hline \multicolumn{6}{|c|}{ Estado de saúde** } \\
\hline $62,2 \pm 18,4$ & $69,0 \pm 21,3$ & 0,225 & $71,7 \pm 19,6$ & $81,1 \pm 15,3$ & 0,086 \\
\hline
\end{tabular}

* Menor pontuação = maior independência ou ausência de problemas

** Maior pontuação = melhor estado de saúde

EQ-5D: Euro Quality of life-5 dimensions; MP: Mobilização precoce; EENM: Estimulação elétrica neuromuscular; UTI: Unidade de terapia intensiva.

Fonte: autoria própria (2021). 
Tabela 15: Valores referentes a escala CAM-ICU. Estão dispostos o número de testes realizados pela equipe de enfermagem durante a internação na UTI e o número de vezes em que os testes deram positivo no grupo MP e no grupo MP+EENM. Não houve diferenças significativas entre os dois grupos.

$$
\text { Teste de detecção de delirium: CAM-ICU }
$$

\begin{tabular}{lccc}
\hline Grupos & Número de testes realizados & Número de vezes positivo & $\mathrm{P}$ \\
\hline MP & 198 & 118 & 0,183 \\
MP + EENM & 129 & 98 &
\end{tabular}

CAM-ICU: Confusion Assessment Method in Intensive Care Unit; MP: Mobilização precoce; EENM: Estimulação elétrica neuromuscular.

Fonte: autoria própria (2021). 


\section{Discussão}

Em nosso conhecimento, este é o primeiro estudo prospectivo e randomizado que delineou como desfecho primário a avaliação da funcionalidade (obtida pela escala FSS-ICU) de pacientes internados em UTI que foram submetidos à aplicação precoce e adicional da EENM a um protocolo de MP. Os pacientes que realizaram a MP e receberam a EENM apresentaram melhores desfechos funcionais no primeiro dia consciente, na alta da UTI e na alta hospitalar e levaram menos tempo para se levantar pela primeira vez durante a internação na UTI. Eles também tiveram um menor tempo de internação hospitalar, menor incidência de ICU-AW e maior força muscular nos três momentos avaliados. Adicionalmente, não houve eventos adversos relacionados aos protocolos do estudo. Esses resultados demonstram que a realização de EENM associada a MP é benéfica e factível de ser realizada em pacientes críticos.

\subsection{População do estudo}

Incialmente, avaliamos as características clínicas e demográficas, que foram semelhantes nos dois grupos estudados. Isto reforça que o processo de randomização foi adequado e os dois grupos, equiparáveis. Adicionalmente, os preditores prognósticos e escores de disfunção orgânica como o índice de Charlson, SAPS 3 e SOFA ${ }^{(7)}$ também foram semelhantes entre os dois grupos.

Além disso, considerando que o uso de alguns medicamentos, como sedativos, bloqueadores neuromusculares e drogas vasoativas podem também interferir em desfechos funcionais, mobilidade, incidência de delirium, prognóstico, tempo de internação, e recuperação clínica ${ }^{(20)}$, nós avaliamos os dias de utilização dessas classes medicamentosas, que foi semelhante nos dois grupos. Não foi possível avaliar a dose desses medicamentos, porém considerando que o perfil diagnóstico dos dois grupos é similar e que eles estavam submetidos ao mesmo protocolo de sedação, acreditamos que a dose dos medicamentos seja similar.

\subsection{Aceitabilidade e aplicabilidade dos protocolos}

Todos os pacientes foram monitorados durante as sessões de MP e EENM para identificar possíveis instabilidades fisiológicas. Não ocorreram efeitos adversos decorrentes das sessões de MP. Já em relação à EENM, nenhuma sessão foi interrompida por 
instabilidade clínica e nenhum efeito adverso foi registrado. No entanto, 4 sessões foram interrompidas por intolerância à dor causada pela EENM. Após estas ocorrências, foi instituído que caso o paciente mantivesse queixa de dor, a intensidade da EENM seria diminuída, mas a sessão não seria interrompida ${ }^{(61)}$.

A perda de massa muscular aguda apresenta uma correlação negativa com o tempo de internação e parece ser maior nas primeiras 2-3 semanas de imobilização/internação na UTI ${ }^{(25)}$, que é também o momento em que grande parte dos pacientes passa por períodos de sedação ${ }^{(94)}$. Esta diminuição da massa muscular pode ocorrer de forma substancial em apenas 5 dias de imobilização ${ }^{(95)}$ por isso, é importante desenvolver estratégias intervencionistas para atenuar a atrofia muscular durante este período de desuso ${ }^{(59)}$.

Ainda não existe um consenso sobre o melhor momento para se iniciar a MP, porém considerando as características da perda muscular no paciente crítico, uma metaanálise sugere que o ideal seria iniciar a mobilização entre 48 a 72 horas da instituição da ventilação mecânica $^{(38)}$. Em ambos os grupos, o protocolo de MP iniciou-se no primeiro dia de internação na UTI conforme planejado, visto que todos os pacientes foram admitidos já intubados e sob ventilação mecânica.

A introdução de algum nível de contração muscular pela aplicação da estimulação elétrica neuromuscular (EENM) somada a um suporte nutricional adequado pode aumentar a síntese proteica muscular e prevenir ou atenuar a perda muscular a curto prazo em várias populações clínicas ${ }^{(59)}$. Sendo assim, uma das vantagens da associação da EENM à MP em relação a MP isolada é que o paciente não necessita estar desperto e consciente para realizar esta terapia ${ }^{(70)}$, favorecendo assim a possibilidade de aplicação o mais cedo possível após a admissão. Por estes motivos, no grupo MP+EENM, a eletroestimulação iniciou-se dentro de uma média de 34 horas de internação na UTI.

Em nosso estudo, o tempo de terapia foi estipulado em 60 minutos com base em estudos prévios $^{(72,74)}$. Este é outro fator que pode influenciar nos benefícios da EENM, visto que quando esta terapia foi aplicada por menos tempo, não resultou em melhor força muscular e melhor estado funcional ${ }^{(77)}$.

Para avaliar eventos adversos, antes e após as sessões de EENM, foram coletadas informações referentes aos dados vitais, que se mantiveram estáveis, reforçando que a EENM é uma terapia segura. Nós observamos apenas uma variação na $\mathrm{SpO}_{2}$, que apresentou um leve aumento na média entre antes e depois de cada intervenção (aproximadamente 0,30 pontos), o que é não tem impacto clínico. Este discreto aumento pode ter sido causado não diretamente pela ação da EENM, mas pelo fato de que muitas vezes o paciente era acordado para a 
realização da terapia além do estímulo elétrico causar um leve desconforto, de forma que aumentasse o nível de despertar do paciente. Sergers e colaboradores (2014) já haviam demonstrado que a EENM é uma intervenção segura e que não provoca grandes alterações dos dados vitais durante sua aplicação.

\subsection{Tamanho das amostras}

É importante considerar que cada escala utilizada neste estudo avalia diferentes aspectos da evolução do paciente, com vantagens e limitações. Devido aos diferentes níveis de dificuldade, obtivemos menos pacientes em ambos os grupos avaliados com a escala MRC e a PFIT. Estas escalas apresentam testes de força, o que necessita da cooperação total do paciente. A escala PFIT ainda apresenta um teste de resistência física (marcha estacionária), e embora ela tenha sido criada especialmente para a UTI, isto a torna mais complexa para pacientes gravemente enfermos.

Em contrapartida, a FSS-ICU avalia exclusivamente as atividades funcionais, principalmente com foco nas mudanças de posicionamento, o que a tornou mais adequada para a avaliação da nossa amostra (pacientes críticos graves). Devido a estas diferenças entre as escalas, foram obtidos diferentes tamanhos de amostra em cada desfecho.

\subsection{Desfechos clínicos e funcionais}

Os pacientes que foram submetidos à EENM precoce adicionalmente à MP, comparados com o grupo que foi submetido somente à MP, obtiveram escores mais elevados nas duas escalas funcionais (FSS-ICU e PFIT) e no MRC no primeiro dia acordado, na alta da UTI e na alta hospitalar. Embora alguns estudos também tenham demonstrado que o uso adicional de EENM pode aumentar ou manter a força muscular em pacientes críticos ${ }^{(62,70,72,}$ 74), outros estudos não observaram esses efeitos ${ }^{(77,78,96)}$. A diferença pode estar relacionada ao tempo para início da EENM, período de tratamento e protocolos utilizados. Em nosso estudo, a EENM foi iniciada no segundo dia de admissão à UTI e este pode ser um fator importante para a melhora do estado funcional dos pacientes avaliados.

Embora não haja consenso quanto ao melhor momento para iniciar a eletroestimulação, sabe-se que a perda muscular ocorre cedo e rapidamente durante a primeira semana de doença crítica ${ }^{(32)}$. Como a EENM não requer a cooperação do paciente, pode ser aplicada precocemente, antes do início da perda muscular e com um período de tratamento de mais de 30 minutos diários ${ }^{(70,72,74)}$. 
Alguns estudos não demostraram benefícios da $\operatorname{EENM}^{(77,96,97)}$. Curiosamente, alguns deles não aplicaram a EENM tão cedo como em nosso estudo. Wollersheim et al. $2019^{(77)}$ iniciaram a intervenção com EENM 72 horas após a admissão na UTI e o grupo que foi estimulado não apresentou melhor força muscular. Kho et al. 2015(96), iniciou a EENM somente em pacientes com 7 dias na UTI ou pelo menos 4 dias em VM, e também não demonstraram melhora da força de membros inferiores na alta hospitalar.

Similar ao presente estudo, Fossat et al. $2018^{(78)}$ iniciou o tratamento por EENM em pacientes críticos com média de 30 horas após a admissão na UTI e não encontrou melhores resultados nos pacientes estimulados. Eles levantaram a hipótese de que os músculos estavam em uma fase catabólica e isso pode ter interferido na eficácia da eletroestimulação ${ }^{(78)}$. Como neste estudo utilizou-se o cicloergômetro adicionalmente à EENM, outra possibilidade é que a combinação das terapias possa ter sobrecarregado os músculos. Mais pesquisas são necessárias para avaliar o melhor momento de início de tratamento, a associação destas terapias e os melhores protocolos de eletroestimulação.

Outro fator importante a se considerar é a população dos diferentes estudos em EENM. No presente estudo, não excluímos pacientes com acidente vascular cerebral e traumatismo cranioencefálico, visto que esses diagnósticos são prevalentes na maioria das UTIs. Como os pacientes neurológicos podem permanecer sedados por um período mais longo, essa população pode se beneficiar com a aplicação precoce da EENM. Routsi et al. $2010^{(74)}$ também incluiu pacientes neurológicos em seu estudo e demonstrou que a EENM preveniu o desenvolvimento de polineuromiopatia de doença crítica e resultou em menor duração do desmame ventilatório. Diferentemente do estudo de Fossat et al. 2018 ${ }^{(78)}$, em que não foram encontrados resultados benéficos no grupo eletroestimulado, mas os pacientes neurológicos foram excluídos.

Em pacientes comatosos, um estudo avaliou o impacto da aplicação unilateral de eletroestimulação duas vezes ao dia no músculo quadríceps de 6 pacientes críticos totalmente sedados por 7 dias $^{(70)}$. Enquanto que na coxa não estimulada houve perdas de fibras musculares tipo 1 e tipo 2 , no lado estimulado não foi encontrado atrofia muscular ${ }^{(70)}$. Diferente do presente estudo, nesta pesquisa os pacientes foram eletroestimulados unilateralmente e o membro inferior contralateral foi utilizado como controle, o que pode ter gerado esta discrepância nos resultados.

Dois outros estudos randomizados realizados com a estimulação de extremidades inferiores em pacientes críticos demonstraram que os grupos estimulados apresentavam uma maior espessura da camada muscular ${ }^{(71,72)}$. No estudo de Gruther et al. $2010^{(71)}$, foi utilizado 
um protocolo similar ao presente estudo, porém os pacientes foram divididos em agudos e crônicos, e esta diferenciação pode ter gerado resultados mais específicos. Já no estudo de Gerovasili et al. $2009^{(72)}$, os pacientes também foram estimulados por um período similar, porém o tempo on era de 12 segundos e o tempo off era de 6 segundos, o que seria praticamente o oposto do protocolo do presente estudo (tempo on 5 segundos e tempo off 10 segundos). Em contraste, um estudo realizado com pacientes com choque séptico observou que EENM realizada por 7 dias consecutivos nos músculos quadríceps não foi capaz de impedir a redução da massa muscular ${ }^{(68)}$. Esta divergência de resultados em pacientes com o mesmo quadro clínico mostra, mais uma vez, a necessidade de mais pesquisas com padronização dos parâmetros instituídos na aplicação da EENM.

Em outro estudo com pacientes sépticos, foram avaliados 16 indivíduos que foram estimulados nos músculos bíceps braquial e vasto medial (quadríceps) em um lado do corpo $^{(73)}$. Os resultados mostraram que o lado estimulado apresentou maior força muscular quando comparado ao lado não estimulado ${ }^{(73)}$. Estes achados corroboram com os resultados do presente estudo, em que dentre a heterogeneidade de diagnósticos, cerca de $43-49 \%$ dos pacientes apresentaram sepse na admissão.

Estudos anteriores já demonstraram que quando combinado com um protocolo de exercícios, a EENM pode apresentar desfechos positivos ${ }^{(76)}$. Um ensaio clínico dividiu pacientes críticos em quatro grupos: 1) fisioterapia convencional, 2) exercícios padronizados, 3) EENM e 4) exercícios padronizados + EENM. O grupo que teve a associação das duas técnicas teve uma menor duração do tempo de VM e do tempo de sedação ${ }^{(76)}$. Em nosso estudo, o uso adicional de EENM diminuiu os dias de VM, porém sem diferença estatística, provavelmente devido ao pequeno tamanho da amostra. Outros estudos anteriores já mostraram que a EENM pode acelerar o desmame ventilatório ${ }^{(74,93)}$. Embora tenhamos usado uma duração da sessão de EENM semelhante (60 minutos em nosso estudo vs. 55 minutos no estudo de Routsi et al.), a contração muscular diária em nosso estudo durou aproximadamente $33 \%$ menos (considerando o protocolo de EENM), o que também pode ter influenciado nos efeitos da EENM na $\mathrm{VM}^{(74)}$.

Quanto à ICU-AW, sabe-se que é um preditor de aumento do tempo de VM e de permanência na $\mathrm{UTI}^{(88)}$. No presente estudo, o grupo MP+EENM apresentou menor incidência de ICU-AW, o que corrobora com estudos prévios ${ }^{(74)}$. Estes pacientes tiveram um tempo de internação hospitalar mais curto, o que também é consistente com um estudo piloto que avaliou o efeito da EENM no quadríceps de pacientes críticos ${ }^{(93)}$. Estes achados reforçam o conceito de que a fraqueza muscular adquirida durante a internação pode retardar a 
recuperação do paciente mesmo após a alta da $\mathrm{UTI}^{(27,28)}$, considerando que os pacientes do grupo MP apresentaram um maior tempo de internação hospitalar. Além disso, estes dados demonstram que a resposta à EENM vai além dos grupos musculares diretamente estimulados e se propaga a outros desfechos clínicos.

Não demonstramos diferenças entre os grupos em relação à qualidade de vida, medida pela escala EQ-5D. Outros estudos utilizaram a escala SF-36(98, 99) para medir a influência da MP na qualidade de vida de pacientes críticos, mas também não obtiveram resultados significativos. Além disso, o uso adicional de NMES resultou em melhor estado funcional, mas não foi suficiente para diminuir a incidência de delirium ou melhorar a independência do paciente nas atividades avaliadas pelo índice de Barthel.

Adicionalmente, durante a internação na UTI, a ocorrência de delirium é frequente em pacientes críticos, e pode ser associado a um maior tempo de internação na UTI e hospitalar ${ }^{(100)}$, maior tempo de ventilação mecânica ${ }^{(101)}$ e consequentemente, um aumento da mortalidade ${ }^{(101)}$. Alguns estudos demonstraram uma ligação entre delirium e declínio funcional $^{(100,102,103)}$. Devido a sua associação com desfechos desfavoráveis, é muito importante que sejam definidas intervenções que possam alterar seu curso ${ }^{(104)}$. É recomendado que estas intervenções sejam farmacológicas como a preferência pelo uso de dexmedetomidina em conjunto com despertar diário ou não farmacológicas, como por exemplo, a MP ${ }^{(20)}$. No estudo de Schweickert et al. $2009^{(40)}$, uma estratégia que consistia na interrupção da sedação e a intervenção de fisioterapia e terapia ocupacional nos primeiros dias da doença crítica resultou em melhores resultados funcionais na alta hospitalar e menor duração do delirium. No presente estudo, apesar do uso adicional de EENM ter resultado em um melhor status funcional, não foi suficiente para diminuir a incidência de delirium.

\subsection{Marcos motores e dose de mobilidade}

Em nossa amostra, com algum tipo de suporte, cerca de 56-61\% dos pacientes sobreviventes conseguiram ficar em posição ortostática pelo menos uma vez durante a internação na UTI e cerca de 34-43\% conseguiram deambular, em ambos os grupos. Estes dados são semelhantes ao observado por Burtin e colaboradores ${ }^{(45)}$, em que na alta da UTI, a maioria dos pacientes em ambos os grupos ainda eram incapazes de levantar-se ou andar independentemente. 
Pela análise obtida nas curvas de kaplan-meier, o grupo MP+EENM atingiu o marco motor "ortostatismo" primeiro. Esses dados reforçam a hipótese de que a terapia com EENM pode ter acelerado a recuperação do estado funcional ${ }^{(105)}$.

Apesar dos pacientes do grupo MP+EENM apresentarem maiores valores nas avaliações funcionais e levarem menos dias para ficar em posição ortostática durante a internação na UTI, não foram encontradas diferenças significativas entre os grupos em relação a quantidade de pacientes que atingiram cada marco motor. Isto pode ser explicado pelo fato das escalas funcionais utilizadas (FSS-ICU e PFIT) abordarem os graus de dependência com uma maior sensibilidade em cada uma das tarefas propostas, o que gera resultados mais específicos $^{(49)}$. Como por exemplo, na escala FSS-ICU, o paciente que fica de pé ou se levanta de uma posição sentada sem assistência recebe pontuação 7 , enquanto o paciente que precisa de assistência máxima quando se levanta de uma posição sentada obtém uma pontuação 2. Neste caso, ambos os pacientes ficaram de pé, mas há uma diferença de 5 pontos entre eles quando considerado o grau de independência e a necessidade de suporte, e isso pode impactar diretamente na especificidade dos dados coletados e na qualidade de vida do paciente ${ }^{(49,57,83)}$.

\subsection{Limitações}

Primeiramente, este estudo não foi cego e, portanto, um viés potencial está associado aos médicos, enfermeiros, fisioterapeutas, e outros profissionais de saúde que tratavam dos pacientes em ambos os braços do estudo. Em segundo lugar, o tamanho da amostra e a heterogeneidade diagnóstica dos pacientes não permitiram definir um grupo específico de indivíduos nos quais a associação da MP+EENM seria mais benéfica, apesar de nossos resultados demonstrarem que ela pode ser aplicada em uma UTI geral. Terceiro, não realizamos nenhuma medida não-volitiva para determinar a força muscular, o que pode ter selecionado os pacientes que cooperaram com os testes utilizados.

Apesar destas limitações, este projeto apresentou evidências de que a associação da EENM a um protocolo de MP pode melhorar a força muscular, desfechos funcionais e até mesmo, diminuir os dias de internação hospitalar. Este é o primeiro estudo que mostrou que a associação da EENM a MP pode ser responsável por desfechos funcionais positivos em pacientes críticos. Estudos futuros podem esclarecer os efeitos destas terapias associadas e o quanto podem interferir em desfechos a longo prazo. 


\section{Conclusões}

Concluímos que a aplicação precoce da EENM adicionalmente a um protocolo de MP, a partir do primeiro dia de internação na UTI, promoveu melhores resultados funcionais em pacientes críticos no primeiro dia acordado, na alta da UTI e na alta hospitalar. Adicionalmente, esses pacientes também levaram menos tempo para se levantar pela primeira vez durante a internação na UTI, tiveram um menor tempo de internação hospitalar, menor incidência de ICU-AW e maior força muscular nos três momentos avaliados. Esses resultados demonstram que a realização de EENM associada a MP é benéfica e factível de ser realizada em pacientes críticos. Assim, ainda é necessário o desenvolvimento de grandes ensaios clínicos de alta qualidade nesta área, com o objetivo de propor um padrão de uso destas terapias. 


\section{Referencias}

1. Berthelsen PG, Cronqvist M. The first intensive care unit in the world: Copenhagen 1953. Acta Anaesthesiol Scand. 2003;47(10):1190-5.

2. Weil MH, Tang W. From intensive care to critical care medicine: a historical perspective. Am J Respir Crit Care Med. 2011;183(11):1451-3.

3. Reisner-Sénélar L. The birth of intensive care medicine: Björn Ibsen's records. Intensive Care Med. 2011;37(7):1084-6.

4. Grenvik A, Pinsky MR. Evolution of the intensive care unit as a clinical center and critical care medicine as a discipline. Crit Care Clin. 2009;25(1):239-50, x.

5. Vincent JL, Fink MP, Marini JJ, Pinsky MR, Sibbald WJ, Singer M, et al. Intensive care and emergency medicine: progress over the past 25 years. Chest. 2006;129(4):1061-7.

6. Tobin MJ, Jubran A, Laghi F. Patient-ventilator interaction. Am J Respir Crit Care Med. 2001;163(5):1059-63.

7. Basile M, Press A, Adia AC, Wang JJ, Herman SW, Lester J, et al. Does Calculated Prognostic Estimation Lead to Different Outcomes Compared With Experience-Based Prognostication in the ICU? A Systematic Review. Crit Care Explor. 2019;1(2):e0004.

8. Curtis JR, White DB. Practical guidance for evidence-based ICU family conferences. Chest. 2008;134(4):835-43.

9. Knaus WA, Zimmerman JE, Wagner DP, Draper EA, Lawrence DE. APACHE-acute physiology and chronic health evaluation: a physiologically based classification system. Crit Care Med. 1981;9(8):591-7.

10. Zimmerman JE, Kramer AA. A history of outcome prediction in the ICU. Curr Opin Crit Care. 2014;20(5):550-6.

11. Knaus WA, Draper EA, Wagner DP, Zimmerman JE. APACHE II: a severity of disease classification system. Crit Care Med. 1985;13(10):818-29.

12. Charlson ME, Pompei P, Ales KL, MacKenzie CR. A new method of classifying prognostic comorbidity in longitudinal studies: development and validation. J Chronic Dis. 1987;40(5):373-83.

13. Charlson M, Szatrowski TP, Peterson J, Gold J. Validation of a combined comorbidity index. J Clin Epidemiol. 1994;47(11):1245-51.

14. Le Gall JR, Loirat P, Alperovitch A, Glaser P, Granthil C, Mathieu D, et al. A simplified acute physiology score for ICU patients. Crit Care Med. 1984;12(11):975-7.

15. Moreno RP, Metnitz PG, Almeida E, Jordan B, Bauer P, Campos RA, et al. SAPS 3-From evaluation of the patient to evaluation of the intensive care unit. Part 2: Development of a prognostic model for hospital mortality at ICU admission. Intensive Care Med. 2005;31(10):1345-55.

16. Singer M, Deutschman CS, Seymour CW, Shankar-Hari M, Annane D, Bauer M, et al. The Third International Consensus Definitions for Sepsis and Septic Shock (Sepsis-3). JAMA. 2016;315(8):801-10.

17. Vincent JL, Moreno R, Takala J, Willatts S, De Mendonça A, Bruining H, et al. The SOFA (Sepsis-related Organ Failure Assessment) score to describe organ dysfunction/failure. On behalf of the Working Group on Sepsis-Related Problems of the European Society of Intensive Care Medicine. Intensive Care Med. 1996;22(7):707-10.

18. Ferreira FL, Bota DP, Bross A, Mélot C, Vincent JL. Serial evaluation of the SOFA score to predict outcome in critically ill patients. JAMA. 2001;286(14):1754-8.

19. Jacobi J, Fraser GL, Coursin DB, Riker RR, Fontaine D, Wittbrodt ET, et al. Clinical practice guidelines for the sustained use of sedatives and analgesics in the critically ill adult. Crit Care Med. 2002;30(1):119-41. 
20. Barr J, Fraser GL, Puntillo K, Ely EW, Gélinas C, Dasta JF, et al. Clinical practice guidelines for the management of pain, agitation, and delirium in adult patients in the intensive care unit. Crit Care Med. 2013;41(1):263-306.

21. Kollef MH, Levy NT, Ahrens TS, Schaiff R, Prentice D, Sherman G. The use of continuous i.v. sedation is associated with prolongation of mechanical ventilation. Chest. 1998;114(2):541-8.

22. Hughes CG, McGrane S, Pandharipande PP. Sedation in the intensive care setting. Clin Pharmacol. 2012;4:53-63.

23. Preiser JC, Ichai C, Orban JC, Groeneveld AB. Metabolic response to the stress of critical illness. Br J Anaesth. 2014;113(6):945-54.

24. Brower RG. Consequences of bed rest. Crit Care Med. 2009;37(10 Suppl):S422-8.

25. Gruther W, Benesch T, Zorn C, Paternostro-Sluga T, Quittan M, Fialka-Moser V, et al. Muscle wasting in intensive care patients: ultrasound observation of the M. quadriceps femoris muscle layer. J Rehabil Med. 2008;40(3):185-9.

26. Choi J, Hoffman LA, Schulz R, Tate JA, Donahoe MP, Ren D, et al. Self-reported physical symptoms in intensive care unit (ICU) survivors: pilot exploration over four months post-ICU discharge. J Pain Symptom Manage. 2014;47(2):257-70.

27. Herridge MS, Cheung AM, Tansey CM, Matte-Martyn A, Diaz-Granados N, Al-Saidi $\mathrm{F}$, et al. One-year outcomes in survivors of the acute respiratory distress syndrome. N Engl $\mathbf{J}$ Med. 2003;348(8):683-93.

28. Dinglas VD, Aronson Friedman L, Colantuoni E, Mendez-Tellez PA, Shanholtz CB, Ciesla ND, et al. Muscle Weakness and 5-Year Survival in Acute Respiratory Distress Syndrome Survivors. Crit Care Med. 2017.

29. Vanhorebeek I, Latronico N, Van den Berghe G. ICU-acquired weakness. Intensive Care Med. 2020;46(4):637-53.

30. Stevens RD, Marshall SA, Cornblath DR, Hoke A, Needham DM, de Jonghe B, et al. A framework for diagnosing and classifying intensive care unit-acquired weakness. Crit Care Med. 2009;37(10 Suppl):S299-308.

31. Latronico N, Bolton CF. Critical illness polyneuropathy and myopathy: a major cause of muscle weakness and paralysis. Lancet Neurol. 2011;10(10):931-41.

32. Puthucheary ZA, Rawal J, McPhail M, Connolly B, Ratnayake G, Chan P, et al. Acute skeletal muscle wasting in critical illness. JAMA. 2013;310(15):1591-600.

33. Dos Santos C, Hussain SN, Mathur S, Picard M, Herridge M, Correa J, et al. Mechanisms of Chronic Muscle Wasting and Dysfunction after an Intensive Care Unit Stay. A Pilot Study. Am J Respir Crit Care Med. 2016;194(7):821-30.

34. Vincent JL. The continuum of critical care. Crit Care. 2019;23(Suppl 1):122.

35. Renton J, Pilcher DV, Santamaria JD, Stow P, Bailey M, Hart G, et al. Factors associated with increased risk of readmission to intensive care in Australia. Intensive Care Med. 2011;37(11):1800-8.

36. Bernhardt J, English C, Johnson L, Cumming TB. Early mobilization after stroke: early adoption but limited evidence. Stroke. 2015;46(4):1141-6.

37. Bailey P, Thomsen GE, Spuhler VJ, Blair R, Jewkes J, Bezdjian L, et al. Early activity is feasible and safe in respiratory failure patients. Crit Care Med. 2007;35(1):139-45.

38. Ding N, Zhang Z, Zhang C, Yao L, Yang L, Jiang B, et al. What is the optimum time for initiation of early mobilization in mechanically ventilated patients? A network metaanalysis. PLoS One. 2019;14(10):e0223151.

39. Moss M, Nordon-Craft A, Malone D, Van Pelt D, Frankel SK, Warner ML, et al. A Randomized Trial of an Intensive Physical Therapy Program for Patients with Acute Respiratory Failure. Am J Respir Crit Care Med. 2016;193(10):1101-10. 
40. Schweickert WD, Pohlman MC, Pohlman AS, Nigos C, Pawlik AJ, Esbrook CL, et al. Early physical and occupational therapy in mechanically ventilated, critically ill patients: a randomised controlled trial. Lancet. 2009;373(9678):1874-82.

41. Morris PE, Goad A, Thompson C, Taylor K, Harry B, Passmore L, et al. Early intensive care unit mobility therapy in the treatment of acute respiratory failure. Crit Care Med. 2008;36(8):2238-43.

42. Bernhardt J, Dewey H, Thrift A, Collier J, Donnan G. A very early rehabilitation trial for stroke (AVERT): phase II safety and feasibility. Stroke. 2008;39(2):390-6.

43. Thompson S, Clark A, Molzahn A, Klarenbach S, Tonelli M. Increasing the uptake of exercise programs in the dialysis unit: a protocol for a realist synthesis. Syst Rev. 2016;5:67.

44. Schaller SJ, Anstey M, Blobner M, Edrich T, Grabitz SD, Gradwohl-Matis I, et al. Early, goal-directed mobilisation in the surgical intensive care unit: a randomised controlled trial. Lancet. 2016;388(10052):1377-88.

45. Burtin C, Clerckx B, Robbeets C, Ferdinande P, Langer D, Troosters T, et al. Early exercise in critically ill patients enhances short-term functional recovery. Crit Care Med. 2009;37(9):2499-505.

46. Camargo Pires-Neto R, Fogaça Kawaguchi YM, Sayuri Hirota A, Fu C, Tanaka C, Caruso P, et al. Very early passive cycling exercise in mechanically ventilated critically ill patients: physiological and safety aspects--a case series. PLoS One. 2013;8(9):e74182.

47. Needham DM, Korupolu R, Zanni JM, Pradhan P, Colantuoni E, Palmer JB, et al. Early physical medicine and rehabilitation for patients with acute respiratory failure: a quality improvement project. Arch Phys Med Rehabil. 2010;91(4):536-42.

48. Bruce B, Fries JF, Ambrosini D, Lingala B, Gandek B, Rose M, et al. Better assessment of physical function: item improvement is neglected but essential. Arthritis Res Ther. 2009;11(6):R191.

49. Parry SM, Denehy L, Beach LJ, Berney S, Williamson HC, Granger CL. Functional outcomes in ICU - what should we be using? - an observational study. Crit Care. 2015;19:127.

50. Parry SM, Granger CL, Berney S, Jones J, Beach L, El-Ansary D, et al. Assessment of impairment and activity limitations in the critically ill: a systematic review of measurement instruments and their clinimetric properties. Intensive Care Med. 2015;41(5):744-62.

51. Denehy L, de Morton NA, Skinner EH, Edbrooke L, Haines K, Warrillow S, et al. A physical function test for use in the intensive care unit: validity, responsiveness, and predictive utility of the physical function ICU test (scored). Phys Ther. 2013;93(12):1636-45.

52. Corner EJ, Wood H, Englebretsen C, Thomas A, Grant RL, Nikoletou D, et al. The Chelsea critical care physical assessment tool (CPAx): validation of an innovative new tool to measure physical morbidity in the general adult critical care population; an observational proof-of-concept pilot study. Physiotherapy. 2013;99(1):33-41.

53. Nawa RK, Lettvin C, Winkelman C, Evora PR, Perme C. Initial interrater reliability for a novel measure of patient mobility in a cardiovascular intensive care unit. J Crit Care. 2014;29(3):475.e1-5.

54. Kasotakis G, Schmidt U, Perry D, Grosse-Sundrup M, Benjamin J, Ryan C, et al. The surgical intensive care unit optimal mobility score predicts mortality and length of stay. Crit Care Med. 2012;40(4):1122-8.

55. Hodgson C, Needham D, Haines K, Bailey M, Ward A, Harrold M, et al. Feasibility and inter-rater reliability of the ICU Mobility Scale. Heart Lung. 2014;43(1):19-24.

56. Zanni JM, Korupolu R, Fan E, Pradhan P, Janjua K, Palmer JB, et al. Rehabilitation therapy and outcomes in acute respiratory failure: an observational pilot project. J Crit Care. 2010;25(2):254-62. 
57. Thrush A, Rozek M, Dekerlegand JL. The clinical utility of the functional status score for the intensive care unit (FSS-ICU) at a long-term acute care hospital: a prospective cohort study. Phys Ther. 2012;92(12):1536-45.

58. Dubb R, Nydahl P, Hermes C, Schwabbauer N, Toonstra A, Parker AM, et al. Barriers and Strategies for Early Mobilization of Patients in Intensive Care Units. Ann Am Thorac Soc. 2016;13(5):724-30.

59. Dirks ML, Wall BT, van Loon LJC. Interventional strategies to combat muscle disuse atrophy in humans: focus on neuromuscular electrical stimulation and dietary protein. $\mathrm{J}$ Appl Physiol (1985). 2018;125(3):850-61.

60. Maffiuletti NA. Physiological and methodological considerations for the use of neuromuscular electrical stimulation. Eur J Appl Physiol. 2010;110(2):223-34.

61. Segers J, Hermans G, Bruyninckx F, Meyfroidt G, Langer D, Gosselink R. Feasibility of neuromuscular electrical stimulation in critically ill patients. J Crit Care. 2014;29(6):10828.

62. Wageck B, Nunes GS, Silva FL, Damasceno MC, de Noronha M. Application and effects of neuromuscular electrical stimulation in critically ill patients: systematic review. Med Intensiva. 2014;38(7):444-54.

63. Sillen MJ, Franssen FM, Gosker HR, Wouters EF, Spruit MA. Metabolic and structural changes in lower-limb skeletal muscle following neuromuscular electrical stimulation: a systematic review. PLoS One. 2013;8(9):e69391.

64. Vanderthommen M, Duteil S, Wary C, Raynaud JS, Leroy-Willig A, Crielaard JM, et al. A comparison of voluntary and electrically induced contractions by interleaved $1 \mathrm{H}-$ and 31P-NMRS in humans. J Appl Physiol (1985). 2003;94(3):1012-24.

65. Grunow JJ, Goll M, Carbon NM, Liebl ME, Weber-Carstens S, Wollersheim T. Differential contractile response of critically ill patients to neuromuscular electrical stimulation. Crit Care. 2019;23(1):308.

66. Stefanou C, Karatzanos E, Mitsiou G, Psarra K, Angelopoulos E, Dimopoulos S, et al. Neuromuscular electrical stimulation acutely mobilizes endothelial progenitor cells in critically ill patients with sepsis. Ann Intensive Care. 2016;6(1):21.

67. Smith GV, Alon G, Roys SR, Gullapalli RP. Functional MRI determination of a doseresponse relationship to lower extremity neuromuscular electrical stimulation in healthy subjects. Exp Brain Res. 2003;150(1):33-9.

68. Poulsen JB, Møller K, Jensen CV, Weisdorf S, Kehlet H, Perner A. Effect of transcutaneous electrical muscle stimulation on muscle volume in patients with septic shock. Crit Care Med. 2011;39(3):456-61.

69. Trethewey SP, Brown N, Gao F, Turner AM. Interventions for the management and prevention of sarcopenia in the critically ill: A systematic review. J Crit Care. 2019;50:28795.

70. Dirks ML, Hansen D, Van Assche A, Dendale P, Van Loon LJ. Neuromuscular electrical stimulation prevents muscle wasting in critically ill comatose patients. Clin Sci (Lond). 2015;128(6):357-65.

71. Gruther W, Kainberger F, Fialka-Moser V, Paternostro-Sluga T, Quittan M, Spiss C, et al. Effects of neuromuscular electrical stimulation on muscle layer thickness of knee extensor muscles in intensive care unit patients: a pilot study. J Rehabil Med. 2010;42(6):5937.

72. Gerovasili V, Stefanidis K, Vitzilaios K, Karatzanos E, Politis P, Koroneos A, et al. Electrical muscle stimulation preserves the muscle mass of critically ill patients: a randomized study. Crit Care. 2009;13(5):R161. 
73. Rodriguez PO, Setten M, Maskin LP, Bonelli I, Vidomlansky SR, Attie S, et al. Muscle weakness in septic patients requiring mechanical ventilation: protective effect of transcutaneous neuromuscular electrical stimulation. J Crit Care. 2012;27(3):319.e1-8.

74. Routsi C, Gerovasili V, Vasileiadis I, Karatzanos E, Pitsolis T, Tripodaki E, et al. Electrical muscle stimulation prevents critical illness polyneuromyopathy: a randomized parallel intervention trial. Crit Care. 2010;14(2):R74.

75. Abdellaoui A, Préfaut C, Gouzi F, Couillard A, Coisy-Quivy M, Hugon G, et al. Skeletal muscle effects of electrostimulation after COPD exacerbation: a pilot study. Eur Respir J. 2011;38(4):781-8.

76. Dos Santos FV, Cipriano G, Vieira L, Güntzel Chiappa AM, Cipriano GBF, Vieira P, et al. Neuromuscular electrical stimulation combined with exercise decreases duration of mechanical ventilation in ICU patients: A randomized controlled trial. Physiother Theory Pract. 2018:1-9.

77. Wollersheim T, Grunow JJ, Carbon NM, Haas K, Malleike J, Ramme SF, et al. Muscle wasting and function after muscle activation and early protocol-based physiotherapy: an explorative trial. J Cachexia Sarcopenia Muscle. 2019;10(4):734-47.

78. Fossat G, Baudin F, Courtes L, Bobet S, Dupont A, Bretagnol A, et al. Effect of InBed Leg Cycling and Electrical Stimulation of the Quadriceps on Global Muscle Strength in Critically Ill Adults: A Randomized Clinical Trial. JAMA. 2018;320(4):368-78.

79. França E, Ferrari F, Fernandes P, Cavalcanti R, Duarte A, Martinez B, et al. Fisioterapia em pacientes críticos adultos: recomendações do Departamento de Fisioterapia da Associação de Medicina Intensiva Brasileira. Physical therapy in critically ill adult patients: recommendations from the Brazilian Association of Intensive Care Medicine Department of Physical Therapy. Rev Bras Ter Intensiva2012. p. 6-22.

80. Vivodtzev I, Lacasse Y, Maltais F. Neuromuscular electrical stimulation of the lower limbs in patients with chronic obstructive pulmonary disease. J Cardiopulm Rehabil Prev. 2008;28(2):79-91.

81. Ely EW, Inouye SK, Bernard GR, Gordon S, Francis J, May L, et al. Delirium in mechanically ventilated patients: validity and reliability of the confusion assessment method for the intensive care unit (CAM-ICU). JAMA. 2001;286(21):2703-10.

82. Ely EW, Margolin R, Francis J, May L, Truman B, Dittus R, et al. Evaluation of delirium in critically ill patients: validation of the Confusion Assessment Method for the Intensive Care Unit (CAM-ICU). Crit Care Med. 2001;29(7):1370-9.

83. Silva VZMD, Araújo JA, Cipriano G, Pinedo M, Needham DM, Zanni JM, et al. Brazilian version of the Functional Status Score for the ICU: translation and cross-cultural adaptation. Rev Bras Ter Intensiva. 2017;29(1):34-8.

84. Nordon-Craft A, Schenkman M, Edbrooke L, Malone DJ, Moss M, Denehy L. The physical function intensive care test: implementation in survivors of critical illness. Phys Ther. 2014;94(10):1499-507.

85. De Jonghe B, Bastuji-Garin S, Durand MC, Malissin I, Rodrigues P, Cerf C, et al. Respiratory weakness is associated with limb weakness and delayed weaning in critical illness. Crit Care Med. 2007;35(9):2007-15.

86. Kleyweg RP, van der Meché FG, Schmitz PI. Interobserver agreement in the assessment of muscle strength and functional abilities in Guillain-Barré syndrome. Muscle Nerve. 1991;14(11):1103-9.

87. Tipping CJ, Harrold M, Holland A, Romero L, Nisbet T, Hodgson CL. The effects of active mobilisation and rehabilitation in ICU on mortality and function: a systematic review. Intensive Care Med. 2017;43(2):171-83. 
88. De Jonghe B, Sharshar T, Lefaucheur JP, Authier FJ, Durand-Zaleski I, Boussarsar M, et al. Paresis acquired in the intensive care unit: a prospective multicenter study. JAMA. 2002;288(22):2859-67.

89. Silveira LTYD, Silva JMD, Soler JMP, Sun CYL, Tanaka C, Fu C. Assessing functional status after intensive care unit stay: the Barthel Index and the Katz Index. Int $\mathbf{J}$ Qual Health Care. 2018;30(4):265-70.

90. Ferreira PL, Ferreira LN, Pereira LN. [Contribution for the validation of the Portuguese version of EQ-5D]. Acta Med Port. 2013;26(6):664-75.

91. Pinto EB, Maso I, Vilela RN, Santos LC, Oliveira-Filho J. Validation of the EuroQol quality of life questionnaire on stroke victims. Arq Neuropsiquiatr. 2011;69(2B):320-3.

92. Fischer A, Spiegl M, Altmann K, Winkler A, Salamon A, Themessl-Huber M, et al. Muscle mass, strength and functional outcomes in critically ill patients after cardiothoracic surgery: does neuromuscular electrical stimulation help? The Catastim 2 randomized controlled trial. Crit Care. 2016;20:30.

93. Leite MA, Osaku EF, Albert J, Costa CRLM, Garcia AM, Czapiesvski FDN, et al. Effects of Neuromuscular Electrical Stimulation of the Quadriceps and Diaphragm in Critically Ill Patients: A Pilot Study. Crit Care Res Pract. 2018;2018:4298583.

94. Jackson DL, Proudfoot CW, Cann KF, Walsh T. A systematic review of the impact of sedation practice in the ICU on resource use, costs and patient safety. Crit Care. 2010;14(2):R59.

95. Wall BT, Dirks ML, Snijders T, Senden JM, Dolmans J, van Loon LJ. Substantial skeletal muscle loss occurs during only 5 days of disuse. Acta Physiol (Oxf). 2014;210(3):600-11.

96. Kho ME, Truong AD, Zanni JM, Ciesla ND, Brower RG, Palmer JB, et al. Neuromuscular electrical stimulation in mechanically ventilated patients: a randomized, sham-controlled pilot trial with blinded outcome assessment. J Crit Care. 2015;30(1):32-9.

97. Patsaki I, Gerovasili V, Sidiras G, Karatzanos E, Mitsiou G, Papadopoulos E, et al. Effect of neuromuscular stimulation and individualized rehabilitation on muscle strength in Intensive Care Unit survivors: A randomized trial. J Crit Care. 2017;40:76-82.

98. Morris PE, Berry MJ, Files DC, Thompson JC, Hauser J, Flores L, et al. Standardized Rehabilitation and Hospital Length of Stay Among Patients With Acute Respiratory Failure: A Randomized Clinical Trial. JAMA. 2016;315(24):2694-702.

99. Kayambu G, Boots R, Paratz J. Early physical rehabilitation in intensive care patients with sepsis syndromes: a pilot randomised controlled trial. Intensive Care Med. 2015;41(5):865-74.

100. Lin SM, Liu CY, Wang CH, Lin HC, Huang CD, Huang PY, et al. The impact of delirium on the survival of mechanically ventilated patients. Crit Care Med. 2004;32(11):2254-9.

101. Ely EW, Shintani A, Truman B, Speroff T, Gordon SM, Harrell FE, et al. Delirium as a predictor of mortality in mechanically ventilated patients in the intensive care unit. JAMA. 2004;291(14):1753-62.

102. Hopkins RO, Weaver LK, Pope D, Orme JF, Bigler ED, Larson-LOHR V. Neuropsychological sequelae and impaired health status in survivors of severe acute respiratory distress syndrome. Am J Respir Crit Care Med. 1999;160(1):50-6.

103. McCusker J, Cole M, Dendukuri N, Belzile E, Primeau F. Delirium in older medical inpatients and subsequent cognitive and functional status: a prospective study. CMAJ. 2001;165(5):575-83.

104. Pun BT, Ely EW. The importance of diagnosing and managing ICU delirium. Chest. 2007;132(2):624-36. 
105. Zanotti E, Felicetti G, Maini M, Fracchia C. Peripheral muscle strength training in bed-bound patients with COPD receiving mechanical ventilation: effect of electrical stimulation. Chest. 2003;124(1):292-6. 


\title{
ANEXOS
}

\section{ANEXO A - Escala FSS-ICU}

\section{Escala de Estado Funcional em Unidade de Terapia Intensiva (Functional Status Score for the Intensive Care Unit - FSS-ICU) - Tradução Brasileira}

\author{
Recomendações Gerais para Avaliação:
}

A escala FSS-ICU deve ser utilizada para graduar somente o desempenho físico do paciente. $O$ avaliador poderá auxiliar com o manejo de dispositivos médicos e/ou equipamentos, assim como supervisionar os sistemas de monitorização dos pacientes. Esta forma de assistência do avaliador não deverá ser considerada quando graduarmos o desempenho físico do paciente.

- Exceto em casos especificos (como por exemplo, deambulação ou mobilidade por cadeira de rodas, onde para se graduar o desempenho permite-se 2 avaliadores), a graduação da FSS-ICU deve ser baseada em um único avaliador que fisicamente auxilia ou supervisiona o paciente para cada tarefa proposta. A graduação da FSS-ICU deve ser realizada sem o uso de dispositivos que auxiliem o paciente a levantar.

- A graduação deve ser baseada em como a tarefa proposta é executada na sessão em que o teste é realizado, e não como o avaliador observou como o paciente a realizou no passado, ou antecipar se o paciente pode ser capaz de executá-la.

\section{Instrucões Específicas para Graduação:}

Para cada tarefa descrita nas páginas seguintes, passe o valor para a tabela abaixo. Para cada tarefa, os valores mínimo e máximo são 0 e 7, respectivamente. $\mathrm{O}$ "Valor Total" será a soma dos itens 1 a 5 , apresentando um valor mínimo e máximo de 0 e 35, respectivamente.

Se a(s) tarefa(s) não foi realizada por outra razão que não seja a fraqueza (como exemplo recusa do paciente, ou o equipamento médico do paciente evita a realização da tarefa), use o seguinte método para graduar:

1) Se $\leq 2$ tarefas não foram realizadas por essas razões, utilize o valor médio dos itens que foram completados para graduar 1 ou 2 tarefas não realizadas.

2) $\mathrm{Se}>2$ tarefas não foram realizadas por essas razões, somente as tarefas completadas poderão ser graduadas e o valor total da FSS-ICU não poderá ser calculado.

\begin{tabular}{|c|c|}
\hline Tarefa & Valor \\
\hline 1. Rolar & \\
\hline $\begin{array}{l}\text { 2. Transferência da posição } \\
\text { supina para sentada }\end{array}$ & \\
\hline $\begin{array}{l}\text { 3. Transferência da posição } \\
\text { sentada para posição de pé }\end{array}$ & \\
\hline 4. Sentar na beira da cama & \\
\hline 5. Andar & \\
\hline Valor Total (soma dos valores) & \\
\hline
\end{tabular}

\section{(c) $(-)$}

Este trabalho, criado por Vinicius Z. Maldaner da Silva, PT, PhD; Fernando S. Guimaraes PT, PhD; Dale M. Needham MD, PhD; Jennifer M. Zanni, PT, PhD; Mariela Pinedo, BA; e Johns Hopkins University Outcomes Group after Critical Illness \& Surgery (OACIS) Group, está licenciado sob uma Licença Atribuição-Não Comercial-Sem Derivações 4.0 Internacional. Para ver uma cópia desta licença visite http://creativecommons.org/licenses/by-nc-nd/4.0/deed.pt_BR. 


\section{Descricões}

\section{1 - Rolar}

\section{O paciente precisa de ajuda para se virar na cama?}

[Nota: Coloque a cama tão plana quanto seguramente possivel para o paciente]

Não:

$>$ O paciente consegue rolar sem usar a grade da cama ou outro objeto para puxar? Se sim, marque 7.

O paciente precisa da grade da cama ou algum objeto para rolar na cama? Se sim, marque 6.

Sim:

> O paciente precisa de incentivo verbal ou orientação para rolar, mas é fisicamente capaz de rolar na cama sem assistência (o paciente pode utilizar a grade da cama ou algum objeto como ajuda caso necessário)? Se sim, marque 5 .

> $\mathrm{O}$ paciente requer assistência mínima (definida como um paciente que realiza $75 \%$ ou mais do trabalho total) para rolar na cama? Se sim, marque 4.

$>\mathrm{O}$ paciente requer assistência moderada (definida como um paciente que realiza entre $26-74 \%$ do trabalho total) para rolar na cama? Se sim, marque 3.

> paciente requer assistência máxima (definida como um paciente que realiza $25 \%$ ou menos do trabalho total) para rolar na cama? Se sim, marque 2.

O paciente é completamente dependente para se virar (definido como a tarefa "rolar" sendo realizada, mas o paciente não sendo capaz de ajudar em todo o processo)? Se sim, marque 1.

$>$ O paciente é incapaz de tentar ou completar a tarefa "rolar" por fraqueza muscular? Se sim, marque 0.

Se a tarefa não foi realizada por alguma outra razão que não seja fraqueza (como por exemplo, recusa do paciente ou o equipamento médico evita a realização da tarefa), não pontuar.

Este trabalho, criado por Vinicius Z. Maldaner da Silva, PT, PhD; Fernando S. Guimaraes University Outcomes Group after Critical Illness \& Surgery (OACIS) Group, está licenciado sob uma Licença Atribuição-Não Comercial-Sem Derivações 4.0 Internacional. Para ver uma cópia desta licença visite http://creativecommons.org/licenses/by-nc-nd/4.0/deed.pt_BR. 


\section{2 - Transferência da posição supina para a posição sentada:}

\section{O paciente precisa de assistência para se sentar partindo de uma posição deitada?}

[Nota: Coloque a cama tão plana quanto seguramente possivel para o paciente]

Não:

$>$ O paciente consegue ir de uma posição deitada para uma posição sentada sozinho, sem se segurar na grade da cama ou em outro objeto para apoio? Se sim, marque 7 .

O paciente consegue ir de uma posição deitada para uma posição sentada sozinho, mas precisa se segurar na grade da cama ou em outro objeto para apoio? Se sim, marque 6.

Sim:

O paciente precisa de incentivo verbal ou orientação para ir de uma posição deitada para uma posição sentada, apesar de ser fisicamente capaz de realizar a tarefa sem assistência (ele pode usar a grade da cama ou outro objeto para ajudá-lo, se necessário)? Se sim, marque 5.

O paciente precisa de assistência mínima para ir de uma posição deitada para uma posição sentada (definida como um paciente que realiza $75 \%$ ou mais do trabalho total)? Se sim, marque 4.

O paciente precisa de assistência moderada para ir de uma posição deitada para uma posição sentada (definida como um paciente que realiza entre $26-74 \%$ do trabalho total)? Se sim, marque 3.

O paciente precisa de assistência máxima para ir de uma posição deitada para uma posição sentada (definida como o paciente que realiza $25 \%$ ou menos do trabalho total)? Se sim, marque 2.

O paciente é completamente dependente ou incapaz de ajudar no esforço para ir de uma posição deitada para uma posição sentada (definido como a tarefa "transferência de supino para sentado" sendo realizada, mas o paciente não sendo capaz de ajudar em todo o processo)? Se sim, marque 1.

O paciente é incapaz de tentar ou completar a tarefa "transferência de supino para sentado" por fraqueza muscular? Se sim, marque 0.

Se a tarefa não foi realizada por alguma outra razão que não seja fraqueza (como por exemplo, recusa do paciente, ou o equipamento médico do paciente evita a realização da tarefa), não pontuar.

Este trabalho, criado por Vinicius Z. Maldaner da Silva, PT, PhD; Fernando S. Guimaraes PT, PhD; Dale M. Needham MD, PhD; Jennifer M. Zanni, PT, PhD; Mariela Pinedo, BA; e Johns Hopkins University Outcomes Group after Critical Illness \& Surgery (OACIS) Group, está licenciado sob uma Licença Atribuição-Não Comercial-Sem Derivações 4.0 Internacional. Para ver uma cópia desta licença visite http://creativecommons.org/licenses/by-nc-nd/4.0/deed.pt_BR. 


\section{3 - Transferência da posição sentada para a posição de pé:}

\section{O paciente precisa de assistência para ficar de pé partindo de uma posição sentada?}

[Nota: as transferências podem ocorrer partindo de qualquer superfície com altura razoável e habitual, incluindo a cama, cadeira, etc.]

Não:

O paciente fica de pé ou se levanta de uma posição sentada sem usar os braços da cadeira ou um objeto para apoio? Se sim, marque 7.

O paciente precisa dos braços da cadeira ou de um objeto de apoio para se levantar? Se sim, marque 6.

Sim:

O paciente precisa de incentivo verbal ou orientação para se levantar de uma posição sentada, mas pode fazê-lo sem ajuda física (o paciente pode usar os braços da cadeira ou outro objeto para ajudá-lo, se necessário)? Se sim, marque 5.

O paciente precisa de assistência mínima quando se levanta de uma posição sentada (definida como um paciente que realiza $75 \%$ ou mais do trabalho total)? O paciente pode usar os braços da cadeira, se necessário. Se sim, marque 4.

O paciente precisa de assistência moderada quando se levanta de uma posição sentada (definida como um paciente que realiza entre $26-74 \%$ do trabalho total)? Se sim, marque 3 .

O paciente precisa de assistência máxima quando se levanta de uma posição sentada (definida como o paciente realizando $25 \%$ ou menos do trabalho total)? Se sim, marque 2.

$>$ O paciente é completamente dependente ou incapaz de ajudar na tarefa de se levantar de uma posição sentada (definido como a tarefa "transferência da posição sentada para a posição de pé" sendo realizada, mas o paciente não sendo capaz de ajudar em todo o processo)? Se sim, marque 1.

$>$ O paciente é incapaz de tentar ou completar a tarefa "transferência da posição sentada para a posição de pé" por fraqueza muscular? Se sim, marque 0.

Se a tarefa não foi realizada por alguma outra razão que não seja fraqueza (como por exemplo, recusa do paciente, ou o equipamento médico do paciente evita a realização da tarefa), não pontuar.

Este trabalho, criado por Vinicius Z. Maldaner da Silva , PT, PhD; Fernando S. Guimaraes PT, PhD; Dale M. Needham MD, PhD; Jennifer M. Zanni, PT, PhD; Mariela Pinedo, BA; e Johns Hopkins University Outcomes Group after Critical Illness \& Surgery (OACIS) Group, está licenciado sob uma Licença Atribuição-Não Comercial-Sem Derivações 4.0 Internacional. Para ver uma cópia desta licença visite http://creativecommons.org/licenses/by-nc-nd/4.0/deed.pt_BR. 


\section{4 - Sentar na beira da cama:}

\section{O paciente precisa de assistência para sentar na beira da cama?}

[Nota: não há tempo determinado para esta tarefa]

Não:

> O paciente consegue sentar na beira da cama sozinho com suas mãos livres e sem segurar na grade ou em outro objeto para ajuda? Se sim, marque 7.

> paciente precisa usar suas mãos ou a grade da cama para se equilibrar quando senta sozinho na beira da cama? Se sim, marque 6 .

Sim:

> paciente precisa de incentivo verbal ou orientação para sentar na beira da cama, mas é fisicamente capaz de fazê-lo sozinho (pode usar a mão para se equilibrar se necessário)? Se sim, marque 5.

O paciente precisa de assistência mínima para sentar na beira da cama (definido como um paciente que realiza $75 \%$ ou mais do trabalho total)? Se sim, marque 4.

O paciente precisa de assistência moderada para sentar na beira da cama (definido como um paciente que realiza entre $26-74 \%$ do trabalho total)? Se sim, marque 3.

$>$ O paciente precisa de assistência máxima para sentar na beira da cama (definida como o paciente realizando $25 \%$ ou menos do trabalho total)? Se sim, marque 2.

- O paciente é completamente dependente ou incapaz de ajudar para se sentar na beira da cama (definido como a tarefa "sentar na beira da cama" sendo realizada mas o paciente não sendo capaz de ajudar em todo o processo)? Se sim, marque 1.

O paciente é incapaz de tentar ou completar a tarefa "sentar na beira da cama" por fraqueza muscular? Se sim, marque 0 .

Se a tarefa não foi realizada por alguma outra razão que não seja fraqueza (como por exemplo, recusa do paciente, ou o equipamento médico do paciente evita a realização da tarefa), não pontuar.

Este trabalho, criado por Vinicius Z. Maldaner da Silva, PT, PhD; Fernando S. Guimaraes University Outcomes Group after Critical Illness \& Surgery (OACIS) Group, está licenciado sob uma Licença Atribuição-Não Comercial-Sem Derivações 4.0 Internacional. Para ver uma cópia desta licença visite http://creativecommons.org/licenses/by-nc-nd/4.0/deed.pt_BR. 


\section{5- Andar:}

\section{O paciente precisa de ajuda para andar 150 pés $(45 \mathrm{~m})$ ?}

[Nota: Acompanhar o paciente com uma cadeira de rodas ou ajudar com o equipamento médico durante a deambulação não deve ser considerado como uma segunda pessoa auxiliando a tarefa]

Não:

> $\mathrm{O}$ paciente consegue andar 150 pés $(45 \mathrm{~m})$ sem o uso de equipamento de assistência, tais como bengala, andador, muletas ou o uso de apoios ou próteses? Se sim, marque 7.

O paciente consegue andar 150 pés $(45 \mathrm{~m})$, mas requer o uso de equipamento de assistência, tais como bengalas, andador, muletas ou o uso de apoios ou próteses? Se sim, marque 6.

Sim:

O paciente precisa somente de supervisão ou incentivo verbal para andar 150 pés $(45 \mathrm{~m}$ ) sem ajuda física (o paciente pode usar um equipamento de assistência, se necessário)? Se sim, marque 5.

$>$ O paciente consegue andar ao menos 150 pés $(45 \mathrm{~m})$ com assistência mínima de somente uma pessoa (definida como um paciente que realiza $75 \%$ ou mais do esforço para andar)? Se sim, marque 4.

O paciente consegue andar ao menos 150 pés $(45 \mathrm{~m})$ com assistência moderada de somente uma pessoa (definida como um paciente capaz de realizar entre $50 \%$ e $74 \%$ do esforço para andar)? Se sim, marque 3.

O paciente consegue andar ao menos 50 pés (15 metros) com assistência de somente uma pessoa? Se sim, marque 2.

O paciente consegue andar menos de 50 pés (15 metros) com assistência de uma pessoa ou precisa da assistência de duas pessoas para ajudá-lo fisicamente a andar qualquer distância? Se sim, marque 1.

$>$ O paciente é incapaz de tentar ou completar a tarefa "andar" por fraqueza muscular? Se sim, marque 0.

Se a tarefa não foi realizada por alguma outra razão que não seja fraqueza (como por exemplo, recusa do paciente, ou o equipamento médico do paciente evita a realização da tarefa), não pontuar.

\section{(c) (1) $(\Theta$}

Este trabalho, criado por Vinicius Z. Maldaner da Silva, PT, PhD; Fernando S. Guimaraes PT, PhD; Dale M. Needham MD, PhD; Jennifer M. Zanni, PT, PhD; Mariela Pinedo, BA; e Johns Hopkins University Outcomes Group after Critical Illness \& Surgery (OACIS) Group, está licenciado sob uma Licença Atribuição-Não Comercial-Sem Derivações 4.0 Internacional. Para ver uma cópia desta licença visite http://creativecommons.org/licenses/by-nc-nd/4.0/deed.pt_BR. 
Mobilidade com a cadeira de rodas:

\section{O paciente precisa de ajuda para conduzir a cadeira de rodas em uma superfície plana por 150 pés (45 metros)?}

[Nota: Ajudar com o equipamento médico (por exemplo, suporte para medicação intravenosa, monitorização cardíaca, etc.) enquanto o paciente conduz a cadeira de rodas não deve ser considerado como uma segunda pessoa auxiliando a tarefa]

Não:

O paciente pode conduzir a cadeira de rodas de forma segura por 150 pés (45 metros) sem supervisão ou ajuda física? Se sim, marque 6.

Sim:

O paciente requer apenas supervisão ou incentivo verbal para conduzir a cadeira de rodas de forma segura por 150 pés (45 metros) sem ajuda física? Se sim, marque 5 .

- O paciente consegue conduzir a cadeira de rodas de forma segura por no mínimo 150 pés (45 metros) apenas com assistência ocasional mínima (definida como a capacidade do paciente para realizar mais de $75 \%$ da tarefa) de uma pessoa, como por exemplo, para girar ou negociar limites? Se sim, marque 4.

O paciente consegue conduzir a cadeira de rodas de forma segura por no mínimo 150 pés (45 metros) com assistência moderada de apenas uma pessoa (definido como a capacidade do paciente para realizar 50 a $74 \%$ da tarefa)? Se sim, marque 3 .

O paciente consegue conduzir a cadeira de rodas de forma segura por ao menos 50 pés (15 metros) com a assistência de apenas uma pessoa? Se sim, marque 2.

> $\mathrm{O}$ paciente consegue conduzir a cadeira de rodas de forma segura por menos de 50 pés (15 metros) com a assistência de uma pessoa ou requer a assistência de duas pessoas para auxiliar fisicamente a conduzir a cadeira de rodas em qualquer distância? Se sim, marque 1.

$>$ O paciente é incapaz de tentar ou completar a tarefa "mobilidade com a cadeira de rodas" por fraqueza muscular? Se sim, marque 0 .

Se a tarefa não foi realizada por alguma outra razão que não seja fraqueza (como por exemplo, recusa do paciente, ou o equipamento médico do paciente evita a realização da tarefa), não pontuar.

Este trabalho, criado por Vinicius Z. Maldaner da Silva, PT, PhD; Fernando S. Guimaraes PT, PhD; Dale M. Needham MD, PhD; Jennifer M. Zanni, PT, PhD; Mariela Pinedo, BA; e Johns Hopkins University Outcomes Group after Critical Illness \& Surgery (OACIS) Group, está licenciado sob uma Licença Atribuição-Não Comercial-Sem Derivações 4.0 Internacional. Para ver uma cópia desta licença visite http://creativecommons.org/licenses/by-nc-nd/4.0/deed.pt_BR. 
ANEXO B - Escala PFIT

Marcação do Teste de Função Física na unidade de terapia intensiva (PFIT)

O PFIT é uma bateria de medidas de desfechos envolvendo 4 componentes: assi stência do sentar para levantar; cadência da marcha estacionária (marcha no local); força para flexão de ombro e extensão de joelho. O PFIT foi desenvolvido como uma medida de desfecho para avaliar e prescrever a reabilitação em pacientes com doenças críticas. Pacientes estão frequentemente muito doentes e em ambiente impróprio para realizar medidas funcionais como o teste de caminhada de 6 minutos ou teste timed up to go (TUG) e não capaz de responder questões especificas como no índice de Barthel.

\section{Componentes do PFIT:}

- Assistência (sentar para levantar)

- Cadência (passos/minuto)

- Ombro (força de flexão)

- Joelho (força de extensão)

\section{Performance do teste}

A percepção subjetiva do esforço (RPE) avaliada pela escal a modificada de BORG, a saturação de oxigênio (SPO2), frequência cardíaca e pressão arterial media são moni toradas durante o teste e treinamento.

\section{Preparação}

- Colocar o paciente fora do leito (OOB).

- Sentar o paciente na cadeira.

- O paciente deve ser assi sti do se requerido para mover em di reção à bei ra da cadeira para que ele possa ser capaz de tentar ficar em pé.

- Pacientes devem ser informados sobre o que consi ste o teste, e as segui ntes instruções padroni zadas para a marcha no local devam ser lidas.

- “Uma vez que você esteja na posição em pé, nós solicitaremos para marchar no chão. Nós gostaríamosque você marchasseno chão o maior tempo que você puder. Nós iremos gravar quanto tempo você andou e quantos passos você fez. Este teste é desenvolvido para gravar sua capacidade máxima de exercício, portanto é muito importante que você marche no chão pelo mai or tempo que você possivel mente possa.

- Se Apl icável, (i.e reteste), então: “Da última vez que vc fez o teste, você andou por e fez passos.”

- Dê incentivo padronizado a cada 10 segundos. "Continue o máximo que você puder, Você está indo bem, Bem feito”. 
- Se o paciente é incapaz de sentar fora do leito $(\mathrm{OOB})$ o paciente pode fazer o sentar-levantar na bei rada cama. Para padronizar o teste a al tura da cadei ra/cama deverá ser ajustada para que os pés do paciente estejam apoiados no chão com seus joel hos fleti dos a 90 graus. A di stância da cadeira/cama para o chão e o uso de descanso dos braços deverá ser regi strado.

- Solicitar ao paciente para ficar em pé. Nenhuma assi stência deverá ser dada ai menos que o paciente é incapaz de transferir para a posição em pé. Uma pessoa então deverá ajudar, e se isso for insuficiente, a segunda pessoa deverá então assistir (ajudar) a transferência para a posição em pé.

- $\quad$ O nível de assi stência requerida para transferir da posição sentada para em pé deverá ser registrada.

\section{Componente da marcha no local (marcha estacionária) (MOS)}

- Uma vez que o paciente tenha sido assistido para a posição em pé, o componente MOS deverá ser regi strado o mais precoce possivel.

- Instrua o paciente que quando você disser "Comece" você quer que ele marche o mai or tempo possivel antes de col ocá-loem pé.

- Instrua o paciente para "começar" o componente MOS e registre o número de passos dados ( 1 passo = quando o pé do paciente (direito ou esquerdo) entra em contato com o chão) e o tempo gasto (em segundos).

- Uma vez que o paciente parar por mais que 2 segundos (i.e sem novos passos em 2 segundos) o teste deverá ser cessado e o paciente assistido (se requerido) para retornar a posição sentada na cadeira.

- Regi stre aRPE do paciente ao compl etar o componente MOS

- Efeito teto - se fizer 80 passos/minuto - pode parar ou pare depois 5 minutos

\section{Teste de força de membros superiores e inferiores}

- Uma vez que o paciente retornou a posição sentada, o teste de força de flexão de ombro bilateral e extensão de joelho bilateral devem ser real izados uma vez que o paciente tenha tido amplo tempo de descanso.

- Força muscular deve ser graduada de acordo com a escal aOxford 


\begin{tabular}{|l|l|}
\hline $\mathbf{0}$ & Sem contração visivel \\
\hline $\mathbf{1}$ & Tremor de contração (sem movimento do membro) \\
\hline $\mathbf{2}$ & Movimento ativo com eliminação da gravidade \\
\hline $\mathbf{3}$ & Movimento ativo contra a gravidade \\
\hline $\mathbf{4}$ & Movimento ativo contra gravidade e resistência \\
\hline $\mathbf{5}$ & Força Normal \\
\hline
\end{tabular}

- A flexão bilateral de ombro deve ser realizada inicial mente. Movimento ativo livre deve ser aval iado primeiro, e então a resistência será aplicada durante a amplitude de movimento, resistindo no cotovelo onde a resistência é requerida. Força muscular deve ser registrada para cada braço. Registre o maior valor de força marcado na planilha de registro.

- Extensão de joelho deve ser resistida no tornozelo onde a resistência é requeri da para aval iação. Força deve ser regi strada em cada perna.

- A força de flexão de ombro e extensão de joel ho pode ser aval iada com o paciente sentado na cama se o paciente for incapaz de OOB.

\section{Escore da PFIT}

Um escore ordinal é obtido de 12 (ie. Adicione score de 3 para os 4 itens) conforme a Tabela 1 abaixo

Tabela 1: Escore PFIT

\begin{tabular}{|l|l|l|l|l|}
\hline $\begin{array}{l}\text { Assistência } \\
\text { (sentar para } \\
\text { levantar) }\end{array}$ & $\begin{array}{l}\text { Cadência } \\
\text { (Passos por } \\
\text { minuto) }\end{array}$ & $\begin{array}{l}\text { Força no } \\
\text { ombro } \\
\text { (Flexão de } \\
\text { ombro) }\end{array}$ & $\begin{array}{l}\text { Força no joelho } \\
\text { (Extensão de } \\
\text { Joelho) }\end{array}$ & TOTAL \\
\hline 0=incapaz & $0=$ incapaz & $0=$ Grau 0, 1, 2 & $0=$ Grau 0, 1, 2 & \\
\hline 1=assistência x2 & $1=>0-49$ & $1=$ Grau 3 & 1=Grau 3 & \\
\hline 2=assistência $\times 1$ & $2=50-<80$ & 2=Grau 4 & 2=Grau 4 & \\
\hline $\begin{array}{l}3=\text { nenhuma } \\
\text { assistência }\end{array}$ & $3=80+$ & $3=$ Grau 5 & $3=$ Grau 5 & \\
\hline
\end{tabular}


ANEXO C - Escala MRC

Tabela 1 - Escore do Medical Research Council (MRC)

\begin{tabular}{|c|}
\hline Movimentos avaliados \\
\hline - Abduçáo do ombro \\
\hline Flexão do cotovelo \\
\hline Extensão do punho \\
\hline Flexáo do quadril \\
\hline Extensão do joelho \\
\hline Dorsiflexáo do tornozelo \\
\hline Grau de força muscular \\
\hline $0=$ Nenhuma contraçáo visível \\
\hline - 1 = Contraçáo visível sem movimento do segmento \\
\hline - 2 = Movimento ativo com eliminaçáo da gravidade \\
\hline - 3 = Movimento ativo contra a gravidade \\
\hline - 4 = Movimento ativo contra a gravidade e resistência \\
\hline - Forca normal \\
\hline
\end{tabular}

Consiste em seis movimentos avaliados bilaterais e grau de força muscular para cada movimento entre 0 (paralisia total) e 5 (força muscular normal). A pontuaçáo total varia de 0 (tetraparesia completa) a 60 (força muscular normal). Fonte: Adaptado de De Jonghe et al. (2005). ${ }^{(6)}$ 
ANEXO D - Índice de Barthel

\begin{tabular}{|c|c|}
\hline ATIVIDADE & PONTUAÇÃO \\
\hline $\begin{array}{l}\text { ALIM ENTAÇÃO } \\
0=\text { incapacitado } \\
5=\text { precisa de ajuda para cortar, passar manteiga, etc, ou } \\
\text { dieta modificada } \\
10=\text { independente }\end{array}$ & \\
\hline $\begin{array}{l}\text { BANHO } \\
0=\text { dependente } \\
5=\text { independente (ou no chuveiro) }\end{array}$ & \\
\hline $\begin{array}{l}\text { ATIVIDADES ROTINEIRAS } \\
0=\text { precisa de ajuda com a higiene pessoal } \\
5=\text { independente rosto/cabelo/dentes/barbear }\end{array}$ & \\
\hline $\begin{array}{l}\text { VESTIR-SE } \\
0=\text { dependente } \\
5=\text { precisa de ajuda mas consegue fazer uma parte sozinho } \\
10=\text { independente (incluindo botões, zipers, laços, etc.) }\end{array}$ & \\
\hline $\begin{array}{l}\text { INTESTINO } \\
0=\text { incontinente (necessidade de enemas) } \\
5=\text { acidente ocasional } \\
10=\text { continente }\end{array}$ & \\
\hline $\begin{array}{l}\text { SI STEM A URINÁRIO } \\
0=\text { incontinente, ou cateterizado e incapaz de manejo } \\
5=\text { acidente ocasional } \\
10=\text { continente }\end{array}$ & \\
\hline
\end{tabular}




\begin{tabular}{|c|c|}
\hline $\begin{array}{l}\text { USO DO TOILET } \\
0=\text { dependente } \\
5=\text { precisa de al guma ajuda parcial } \\
10=\text { independente ( pentear-se, limpar-se) }\end{array}$ & \\
\hline $\begin{array}{l}\text { TRANSFERÊNCIA (DA CAM A PARA A CADEIRA E } \\
\text { VICE VERSA) } \\
0=\text { incapacitado, sem equil ıbrio para ficar sentado } \\
5=\text { muita ajuda (uma ou duas pessoas, física), pode sentar } \\
10=\text { pouca ajuda (verbal ou física) } \\
15=\text { independente }\end{array}$ & \\
\hline $\begin{array}{l}\text { MOBILIDADE (EM SUPERFICIES PLANAS) } \\
0=\text { imóvel ou < } 50 \text { metros } \\
5=\text { cadeira de rodas independente, incluindo esquinas, }>50 \\
\text { metros } \\
10=\text { caminha com a ajuda de uma pessoa (verbal ou física) > } \\
50 \text { metros } \\
15=\text { independente (mas pode precisar de al guma ajuda; } \\
\text { como exemplo, bengala) }>50 \text { metros }\end{array}$ & \\
\hline $\begin{array}{l}\text { ESCADAS } \\
0=\text { incapacitado } \\
5=\text { precisa de ajuda (verbal }, \text { física, ou ser carregado) } \\
10=\text { independente }\end{array}$ & \\
\hline
\end{tabular}




\section{PONTUAÇÃO}

TOTAL (0-100):

\section{Orientações:}

1. A pontuação na Escala Barthel refere-se ao que os sujeitos fazem e não ao que eles recordam ter feito um dia.

2. Seu principal objetivo é saber sobre o grau de independência em relação a qual quer tipo de ajuda (física ou verbal).

3. Se o sujeito não consegue ler o questionário, alguém pode ler o mesmo para ele. É permito que al gum amigo ou parente responda pelo sujeito (caso este esteja impossibilitado de responder).

4. Preferencialmente procure obter respostas relativas às últimas 48 horas, dependendo do caso, pode ser por períodos maiores.

Observação: esta tradução encontra-se em processo de validação para a língua portuguesa. Data: 28 de agosto de 2006.

\section{Traduzido por:}

Dr. Guanis de Barros Vilela Junior

Grupo de Pesquisas em Qualidade de Vida e Atividade Física

UEPG / METROCAMP 
ANEXO E - Escala EQ-5D

Assinale com uma cruz (assim $\mathbf{~}$ ), um quadrado de cada um dos seguintes grupos, indicando qual das afirmações melhor descreve o seu estado de saúde hoje.

\section{Mobilidade}

Não tenho problemas em andar

Tenho alguns problemas em andar

Estou limitado a ficar na cama

\section{Cuidados Pessoais}

Não tenho problemas com os meus cuidados pessoais

Tenho alguns problemas para me lavar ou me vestir

Sou incapaz de me lavar ou vestir sozinho (a)

Atividades Habituais (ex. trabalho, estudos, atividades domésticas, atividades em familia ou de lazer)

Não tenho problemas em desempenhar as minhas atividades habituais

Tenho alguns problemas em desempenhar as minhas atividades habituais

Sou incapaz de desempenhar as minhas atividades habituais

\section{Dor/Mal-estar}

Não tenho dores ou mal-estar

Tenho dores ou mal-estar moderados

Tenho dores ou mal-estar extremos

\section{Ansiedade/Depressão}

Não estou ansioso(a) ou deprimido(a)

Estou moderadamente ansioso(a) ou deprimido(a)

Estou extremamente ansioso(a) ou deprimido(a) 
O melhor estado de saúde imaginável

Para ajudar as pessoas a dizer quão bom ou mau o seu estado de saúde é nós desenhamos uma escala (semelhante a um termômetro) na qual o melhor estado de saúde que possa imaginar é marcado por 100 e o pior estado de saúde que possa imaginar é marcado por 0 .

Gostaríamos que indicasse nesta escala quão bom ou mau é, na sua opinião, o seu estado de saúde hoje. Por favor, desenhe uma linha a partir do quadrado que se encontra abaixo, até ao ponto da escala que melhor classifica o seu estado de saúde hoje.

\section{O seu estado de saúde




\section{APÊNDICES}

APÊNDICE A - Artigo científico submetido à revista internacional.

Early neuromuscular electrical stimulation in addition to early mobilization improves functional status and decreases hospitalization days of critically ill patients

Débora R. Campos ${ }^{1}$, PT, MSc; Thatiana B. C. Bueno ${ }^{1}$, PT; Jackeline S. G. G. dos Anjos ${ }^{1}$, PT; Daniel Zoppi ${ }^{1}$, MD, PhD; Bruno G. Dantas ${ }^{1}$, MD; Rik Gosselink², PT, PhD; Rinaldo R. de Jesus Guirro $^{3}, \mathrm{PT}, \mathrm{PhD}$; Marcos de Carvalho Borges ${ }^{1}, \mathrm{MD}, \mathrm{PhD}$

${ }^{1}$ Department of Internal Medicine, Ribeirão Preto Medical School, University of São Paulo, Ribeirão Preto, Brazil

${ }^{2}$ Department of Rehabilitation Sciences, Research Group for Rehabilitation in Internal Disorders, KU Leuven, Leuven, Belgium

${ }^{3}$ Department of Health Science, Ribeirão Preto Medical School, University of São Paulo, Ribeirão Preto, Brazil

Name of the institution(s) where the work was performed: Clinical Hospital of Ribeirão Preto, University of São Paulo, Brazil.

This study was financed in part by the Coordenação de Aperfeiçoamento de Pessoal de Nível Superior - Brasil (CAPES) - Finance Code 001.

The authors have not disclosed any potential conflicts of interest.

For information regarding this article, E-mail: marcosborges@fmrp.usp.br. 


\section{ABSTRACT}

Objective: To evaluate the impact of the additional use of early neuromuscular electrical stimulation (NMES) to an early mobilization (EM) protocol.

Design: Randomized controlled trial.

Setting: Intensive care unit (ICU) of the Clinical Hospital of Ribeirão Preto, University of São Paulo, Brazil.

Patients: One hundred and thirty-nine consecutive mechanical ventilated patients were included in the first 48 hours of ICU admission.

Interventions: The patients were divided into two groups: EM and EM+NMES. Both groups received EM daily. In the EM+NMES group, patients received additionally NMES five days a week, per 60 minutes, starting on the first 48 hours of ICU admission until ICU discharge.

Measurements and Main Results: Functional status, muscle strength, ICU and hospital LOS, incidence of delirium, days on mechanical ventilation, mortality and quality of life were assessed. Patients in the EM+NMES group presented a significant higher score of functional status measured by FSS-ICU scale when compared with EM group in the first day awake (21.2 \pm 6.8 vs. $15.2 \pm 8.4, \mathrm{p}=$ $.015)$, ICU discharge ( $26.3 \pm 7.8$ vs. $18.9 \pm 8.6, p=.003)$, and hospital discharge ( $29 \pm 8.8$ vs. $24.5 \pm$ $8.7, p=.014)$, respectively. They also had better functional status measured by the PFIT scale, took less days to stand up during the ICU stay, had a significant shorter hospital LOS, lower incidence of ICU-acquired weakness and better global muscle strength.

Conclusions: The additional application of early NMES promoted better functional status outcomes on the first day awake and at ICU and hospital discharge. The patients on the EM+NMES group also took fewer days to stand up, had shorter hospital LOS, lower incidence of ICU-acquired weakness and better muscle strength. Future studies are still necessary to clarify the effects of associated therapies to EM, specially to assess long-term outcomes.

Key words: critical illness; intensive care units; electrical stimulation; exercise therapy; length of stay; muscle weakness; physical functional performance. 


\section{INTRODUCTION}

Patients in the intensive care unit (ICU) have an increased risk of developing complications related to hospitalization (1). Several conditions, such as ICU-acquired weakness (ICU$A W)$, deep vein thrombosis, cognitive impairments, delirium, nosocomial pneumonia, weaning failure, and mortality, may be associated with prolonged immobilization, mechanical ventilation $(M V)$, and sedation $(2,3)$. The ICU-AW can increase MV duration and the ICU and hospital length of stay (LOS) (4). Muscle wasting is more significant in the first week of a critical illness (5) and is influenced by age, sepsis, systemic inflammation, immobilization, bed rest, longer MV and ICU stay (6). Bed rest affects not only musculoskeletal system, but also cardiovascular and respiratory systems (2).

Early mobilization (EM), structured physical rehabilitation, provided to critically ill patients, usually begins in the first days of hospitalization, and evolves from passive range of motion (ROM) to more complex, functional and resistance exercises (7). EM is a safe and feasible strategy in the ICU (7) that decreases the incidence of delirium $(8,9)$, accelerates functional recovery $(10,11)$, decreases MV duration $(8,12)$, improves the quality of life, and reduces ICU and hospital LOS $(9,11$, 12). However, some studies did not demonstrate these benefits $(13,14)$. The heterogeneity of patient diagnoses, dose of mobility, different EM protocols, and functional scales used may explain the discrepancy in these findings.

Moreover, most critically ill patients, especially during periods of sedation, may not be able to produce active muscle contractions. In this phase, besides passive ROM, it is important to develop additional strategies to prevent the loss of muscle mass such as adequate nutrition, bed cycling, neuromuscular electrical stimulation (NMES), or the combination of these strategies (15).

As NMES does not require the active cooperation of the patient, it is feasible to be applied in the ICU, even in patients under sedation. Based on muscle contractions caused by an electrical impulse (16), NMES can be effective in reducing the loss of muscle mass (17-20) which can also preserve or increase the muscle strength $(21,22)$. In a randomized controlled trial, NMES 
improved physical and functional performance in elderly patients hospitalized with pneumonia (23). A recent metanalysis showed that early implementation of the NMES prevented ICU-acquired weakness, and decreased MV duration, ICU and hospital LOS. However, NMES did not improve patient's functional status during hospitalization (24).

Few studies have evaluated the association of different strategies to prevent ICU-AW and other conditions related to the ICU stay. Therefore, the purpose of this study was to assess the effects of EM associated with NMES in critically ill patients.

\section{MATERIALS AND METHODS}

\section{Study design and randomization}

This randomized controlled trial investigated the additional application of NMES to an EM protocol in critically ill patients. The protocol was approved by the local research ethics committee (CAAE: 72872117.2.0000.5440) and registered in the Brazilian Registry of Clinical Trials (identifier RBR-3w3kmg). Written informed consent was obtained from all patients or their legal representatives.

Consecutive patients admitted in the ICU between July of 2018 and December of 2019 were screened within the first 48 hours. They were randomized with a 1: 1 allocation ratio in two groups and stratified by age (18-29 years, $30-59$ years, and 60 years or more). Data management was performed using the Redcap ${ }^{\circledR}$ platform (Research Electronic Data Capture) and the randomization was done using a computer-generated list (www.sealedenvelope.com). The intervention group received NMES associated with EM (EM+NMES group). The control group was submitted only to the EM protocol (EM group). All patients received respiratory physical therapy.

\section{Study setting and patients}

The research was performed in ICUs of the Emergency Unit of the Ribeirao Preto Clinical Hospital, Ribeirão Preto Medical School, University of São Paulo, with a total of 18 beds for clinical 
and surgical adult patients. Inclusion and exclusion criteria are available at supplemental digital content 1.

\section{Early mobilization protocol}

The same EM protocol was applied in both groups, every day, by the entire ICU multiprofessional staff, coordinated by the physical therapists (supplemental digital content 2). The rehabilitation was guided to achieve daily functional milestones (sitting on the edge of the bed, sitting in the armchair, standing up, and walking). Dose of mobilization was also registered (25).

\section{Neuromuscular electrical stimulation protocol}

The NMES sessions were performed exclusively by one specialized physical therapist, once a day for 60 minutes, five days a week, from ICU admission until ICU discharge. The NMES protocol is available at supplemental digital content 3.

The data collection before and after EM and NMES sessions and the criteria for interrupting the application of the protocols are available at the supplemental digital content 4.

\section{Outcomes}

The following demographic and clinical data were collected: sex, age, comorbidities (assessed with Charlson's comorbidity index) (26), Simplified Acute Physiology Score 3 (SAPS 3) (27), Sequential Organ Failure Assessment (SOFA) (28), ICU and hospital LOS, MV duration, medication used and incidence of delirium (assessed with CAM-ICU scale) (29). Quality of life was evaluated with the Euro Quality of life-5 dimensions and 3 levels questionnaire (EQ-5D-3L) at ICU and hospital discharge and expressed in two items: the EQ-5D-3L index and the Euro Quality visual analogue scale (EQ-VAS) $(30,31)$.

The primary outcome was functional performance evaluated with the Functional Status Score for the ICU (FSS-ICU), applied on the first day awake, at ICU discharge and hospital discharge 
(32, 33). FSS-ICU assesses the hierarchical level of dependency/assistance needed to perform functional mobility tasks in bed, sitting out of bed, standing and walking with a scoring system that rates a functional activity between 1 (total assist) and 7 (complete independence) (33). A score of 0 was assigned if a patient was unable to perform a task, due to either physical limitation or medical status (34).

Secondary outcomes included assessment of muscle strength and functional status with other scales. Medical Research Council Sum-Score $(\mathrm{MRC}-\mathrm{SS})(8,11)$ was used to evaluate global muscle strength on the first day awake, ICU and hospital discharge. ICU-AW was defined as MRC-SS < 48 at ICU discharge. Physical Function Test in the Intensive Care Unit (PFIT) (35) was performed on the first day awake, and at ICU discharge and hospital discharge. The first day awake was defined when the patients could respond to at least three of the following commands ("open/close your eyes", "look at me", "put out your tongue", "nod your head", "raise your eyebrows") (36). The Barthel index (37) was applied to the patient or his/her legal representative on ICU admission to estimate the baseline functional independence and basic activities of daily living (ADL) before the event that caused the hospitalization, and also at ICU and hospital discharge. All the measurements were made by a physical therapist that was specifically trained to apply all the scales and was not involved in the patient's treatment.

\section{Sample size and statistical analysis}

To define differences in functional status between the EM+NMES group and the EM group, we used previous data from our hospital and previous studies $(34,38)$. Considering a minimum change of 7 points in the FSS-ICU scale, assuming a significance level of $5 \%$, a power of $80 \%$ (beta $=20 \%$ ), and 8 points of standard deviation, 21 patients who completed the primary outcome (FSS-ICU score at ICU discharge) were required in each group.

Categorical variables were expressed as percentage. Continuous variables were expressed as mean and standard deviation (normal distribution) and as median and interquartile 
range (non-normal distribution). For categorical variables, the Fisher test was used. For continuous variables with normal distribution, Student's $t$ test was used and for variables with non-normal distribution, the Mann Whitney non-parametric test was used. A value of $p<0.05$ was considered significant. The analysis was performed with GraphPadPrism ${ }^{\circledR}$ and SPSS Statistics ${ }^{\circledR}$.

RESULTS

Patient flow. From July 2018 to December 2019, 451 consecutive patients were screened, 139 patients were enrolled and randomized into the study groups (EM = 69 and EM+NMES $=70$ ). Figure 1 shows the trial flow, and Table 1 shows the baseline characteristics of the enrolled patients.

Baseline Characteristics. There were no significant differences in baseline characteristics, including age, sex, SAPS 3, SOFA, Charlson comorbidity index, body mass index (BMI), Barthel index and diagnoses (Table 1). There were no significant differences in medications such as sedatives, neuromuscular blocking agents, opioids, dexmedetomidine, benzodiazepines, vasoactive drugs and antibiotics (supplemental digital content 5).

Protocol safety and applicability. Mortality rate during ICU stay and hospital stay were similar in both groups: 22 (32\%) deaths in EM group vs. 26 (37\%) in EM+NMES group during the ICU stay $(p=.59)$; and $26(35 \%)$ in EM group vs. $30(42) \%$ in EM+NMES group during the hospital stay ( $p=$ .38). All deaths were unrelated to the interventions in this trial. Forty patients completed the protocol in the EM group and 34 in the EM+NMES group (figure 1). However, 14 patients were unable to perform the FSS-ICU scale in the EM group and 13 in the EM+NMES group. Baseline characteristics (age, sex, SAPS 3, Charlson's comorbidity index, BMI and diagnoses) of these patients were similar. The primary outcome (FSS-ICU at ICU discharge) was assessed in 26 patients in the EM group and in 21 patients in the EM+NMES group.

EM was early initiated in both groups in the first day of ICU admission (15 $\mathrm{h} \pm 8 \mathrm{~h}$ in the EM group vs. $15 \mathrm{~h} \pm 12 \mathrm{~h}$ in the EM+NMES group, $p=.334)$. NMES was initiated mostly in the second 
day of ICU admission ( $34 \pm 12$ hours). A total of 422 NMES sessions were carried out with no significant impact on heart rate, mean arterial pressure and respiratory rate collected before and after these sessions. Transcutaneous oxygen saturation was $96 \%$ (95-98) before NMES sessions and $97 \%$ (95-99) after NMES sessions $(p=.001)$. In $20 \%$ of the NMES sessions, there were no visible muscle contraction. In these sessions, NMES was most affected by edema (49\%), higher doses of vasopressor agents (23\%), use of neuromuscular blockers (16\%), and other causes (12\%). The time of first awakening since ICU admission was 9 (5-13) days on the EM group and 7 (5-10) days on EM+NMES group $(p=.068)$

Primary outcome. Primary outcome results are shown in Figure 2. The EM+NMES group presented a significant higher score of functional status measured by FSS-ICU when compared with EM group in the first day awake ( $21.2 \pm 6.8$ vs. $15.2 \pm 8.4, p=.015)$, ICU discharge ( $26.3 \pm 7.8$ vs. 18.9 $\pm 8.6, p=.003)$, and hospital discharge ( $29 \pm 8.8$ vs. $24.5 \pm 8.7, p=.014)$, respectively.

Secondary outcomes. Functional status and muscle strength were also assessed with PFIT score and MRC-SS. In the first day awake, ICU and hospital discharge, patients that received NMES associated to EM presented a significant improve in the functional status and in the muscle strength, when compared to patients that received only EM (Figure 2). Detailed muscle strength data of lower limbs are available at supplemental digital content 6 . The EM group also had a higher incidence of ICU-AW when comparing to the EM+NMES group: 10 (45\%) vs. $2(11 \%)$ patients, $p=.035$. Primary and secondary outcomes are expressed with their respective effect sizes in the Table 2. Patients in the EM+NMES group took less days to stand up for the first time during ICU stay than patients in the EM group: 8 (5-11) vs. 10 (6-13) days, $p=.036$ (Figure 3).

Of note, hospital LOS was significantly lower in EM+NMES group compared to EM group: 18.5 (10-29) vs. 30 (12-40) respectively, $p=.048$. Barthel index, ICU LOS and days on MV were not significantly different between groups. There were no significant differences between groups regarding EQ-5D-3L index, EQ VAS and CAM-ICU scale. 


\section{DISCUSSION}

The main results of this study were that patients who underwent early NMES associated with EM improved functional recovery, took less days to stand up for the first time, had shorter hospital LOS, and had greater muscle strength on the first day awake, at ICU discharge and hospital discharge. Additionally, this is the first randomized trial that evaluated the association of early NMES and EM in critically ill patients using specific functional scales for the ICU (FSS-ICU and PFIT).

Patients who were submitted to NMES and EM, compared to EM only, had higher scores in both functional scales (FSS-ICU and PFIT) and in the MRC-SS at the first day awake, ICU and hospital discharge. Although some studies have also demonstrated that the additional use of NMES can increase or maintain muscle strength in critically ill patients $(16,22,39)$, other studies did not demonstrate these effects $(18,40,41)$. The difference can be related to the time that NMES was initiated, period of treatment and protocol used. In our study, NMES was initiated at latest in the second day of ICU admission and this might be an important aspect to improve patient's functional status. Moreover, the NMES protocol were well tolerated and presented no adverse effects during the trial, as prior reported $(38,42)$.

Although there is no consensus related to the best time to initiate the NMES therapy, muscle wasting occurs early and rapidly during the first week of critical illness (5). As NMES does not require patient cooperation, it can be applied early, prior of the beginning of muscle wasting and with a treatment period of more than 30 minutes daily $(17,22,39)$. Some studies did not show NMES benefit $(18,40,43)$. Interestingly, some of them did not use NMES as early as our study. Wollersheim et al. (40) started NMES intervention in 72 hours after admission and the group that was stimulated did not present better muscle strength. Kho et al. (18), that started NMES in patients with 7 days in the ICU or 4 days in MV, did not demonstrate improvement in leg strength at hospital discharge.

Similarly to the present study, Fossat et al. 2018 (41) started the NMES treatment in critically ill patients with a median of 30 hours after ICU admission and did not find better results in 
the stimulated patients. They hypothesized that the muscles were in a catabolic phase and this might interfere with NMES therapy (41). As they used bed-cycling in addition to the NMES, another possibility is that therapy combination could have overstressed the muscles. More studies are necessary to evaluate the best timing and protocol for NMES treatment.

Another important factor is the study population of the different trials. We did not exclude patients with stroke and trauma brain injuries, considering that these diagnoses are prevalent in most of ICUs. As neurologic patients may stay sedated for longer period, this population could benefit from early NMES application. Routsi el al. 2010 (22) also included neurological patients in their study and demonstrated that NMES prevented the development of critical illness polyneuromyopathy and resulted in shorter duration of weaning.

Additionally, the moment of initiation and the intensity of EM can also impact in various clinical outcomes $(12,25)$. In our study, both groups were submitted to a structured EM protocol, with similar activities. Of note, patients in the EM+NMES group achieved earlier the milestone "standing up". This data strengthens the hypothesis that the NMES therapy could have accelerated the functional status recovery (44).

It is known that ICU-AW is a predictor of increasing the ICU LOS (4). In the present study, the EM+NMES group had a lower incidence of ICU-AW (defined as MRC sum $<48$ at ICU discharge) corroborating with previous findings (39). Additionally, these patients had a shorter hospital LOS, which is consistent with a previous pilot study that assessed the effect of EM on the quadriceps of critically ill patients (38). This data reinforces the concept that muscle weakness acquired during the ICU stay can delay the patient's recovery even after the ICU discharge $(1,3)$, considering that the patients from the EM group had longer hospital LOS.

In our study, the additional use of NMES decreased the days on MV, however without statistical difference (probably due to the low sample size). Previous studies have already showed that NMES can speed up ventilatory weaning $(22,38)$. Although we used a similar NMES session duration (60 minutes in our study vs. 55 minutes in the study of Routsi et al.), the daily muscle 
contraction in our study lasted approximately $33 \%$ less (considering the NMES protocol), what could also have influenced the effect of NMES on MV (22).

Finally, we did not demonstrate differences between groups regarding EQ-5D-3L index and EQ VAS. Moreover, the additional use of NMES resulted in better functional status, but it was not enough to decrease the incidence of delirium or improve the patient's independency in basic ADL evaluated by the Barthel index.

\section{Clinical implications}

Different from the EM, the early NMES is not well established in the ICU clinical practice (45). Despite some studies demonstrated positive outcomes when applying this preventive strategy, more research is needed to include it in ICU standard care protocols (41). Considering NMES is a feasible and safe technic, it can have a promising future in the treatment of critically ill patients.

\section{Limitations}

This study has some limitations. First, it was not blinded and it was performed at a single site. Second, the sample size and the diagnoses heterogeneity of the patients did not allow to define a specific group of patients that EM+NMES would better benefit, however our results demonstrated that it can be applied in a general ICU. Third, we did not perform any non-volitional measurements to determine the muscle strength which could have selected the patients who were cooperative with the measurements.

\section{Conclusions}

In conclusion, the additional application of early NMES to an EM protocol promoted better functional status outcomes on the first day awake and at ICU and hospital discharge. The EM+NMES group also took fewer days to stand up, had shorter hospital LOS, lower incidence of ICUAW in the patients that were able to perform the MRC-SS and better muscle strength. Future studies 
are still necessary to clarify the effects of associated therapies to EM, specially to assess long-term outcomes.

\section{Acknowledgments}

We thank all the multiprofessional staff from the ICU of the Emergency Unity (Ribeirao Preto Clinical Hospital, Ribeirão Preto Medical School, University of São Paulo) who assisted with data collection and protocol management. We also thank Ricardo Kenji Nawa, PT, PhD, for assisting in the study design.

\section{REFERENCES}

1. Dinglas VD, Aronson Friedman L, Colantuoni E, Mendez-Tellez PA, Shanholtz CB, Ciesla ND, et al. Muscle Weakness and 5-Year Survival in Acute Respiratory Distress Syndrome Survivors. Crit Care Med. 2017.

2. Brower RG. Consequences of bed rest. Crit Care Med. 2009;37(10 Suppl):S422-8.

3. Herridge MS, Cheung AM, Tansey CM, Matte-Martyn A, Diaz-Granados N, Al-Saidi $\mathrm{F}$, et al. One-year outcomes in survivors of the acute respiratory distress syndrome. N Engl $\mathbf{J}$ Med. 2003;348(8):683-93.

4. De Jonghe B, Sharshar T, Lefaucheur JP, Authier FJ, Durand-Zaleski I, Boussarsar M, et al. Paresis acquired in the intensive care unit: a prospective multicenter study. JAMA. 2002;288(22):2859-67.

5. Puthucheary ZA, Rawal J, McPhail M, Connolly B, Ratnayake G, Chan P, et al. Acute skeletal muscle wasting in critical illness. JAMA. 2013;310(15):1591-600.

6. Puthucheary Z, Montgomery H, Moxham J, Harridge S, Hart N. Structure to function: muscle failure in critically ill patients. J Physiol. 2010;588(Pt 23):4641-8.

7. Waldauf P, Jiroutková K, Krajčová A, Puthucheary Z, Duška F. Effects of Rehabilitation Interventions on Clinical Outcomes in Critically Ill Patients: Systematic Review and Meta-Analysis of Randomized Controlled Trials. Crit Care Med. 2020;48(7):1055-65.

8. Schweickert WD, Pohlman MC, Pohlman AS, Nigos C, Pawlik AJ, Esbrook CL, et al. Early physical and occupational therapy in mechanically ventilated, critically ill patients: a randomised controlled trial. Lancet. 2009;373(9678):1874-82.

9. Needham DM, Korupolu R, Zanni JM, Pradhan P, Colantuoni E, Palmer JB, et al. Early physical medicine and rehabilitation for patients with acute respiratory failure: a quality improvement project. Arch Phys Med Rehabil. 2010;91(4):536-42.

10. Burtin C, Clerckx B, Robbeets C, Ferdinande P, Langer D, Troosters T, et al. Early exercise in critically ill patients enhances short-term functional recovery. Crit Care Med. 2009;37(9):2499-505. 
11. Schaller SJ, Anstey M, Blobner M, Edrich T, Grabitz SD, Gradwohl-Matis I, et al. Early, goal-directed mobilisation in the surgical intensive care unit: a randomised controlled trial. Lancet. 2016;388(10052):1377-88.

12. Morris PE, Goad A, Thompson C, Taylor K, Harry B, Passmore L, et al. Early intensive care unit mobility therapy in the treatment of acute respiratory failure. Crit Care Med. 2008;36(8):2238-43.

13. Morris PE, Berry MJ, Files DC, Thompson JC, Hauser J, Flores L, et al. Standardized Rehabilitation and Hospital Length of Stay Among Patients With Acute Respiratory Failure: A Randomized Clinical Trial. JAMA. 2016;315(24):2694-702.

14. Denehy L, Skinner EH, Edbrooke L, Haines K, Warrillow S, Hawthorne G, et al. Exercise rehabilitation for patients with critical illness: a randomized controlled trial with 12 months of follow-up. Crit Care. 2013;17(4):R156.

15. Trethewey SP, Brown N, Gao F, Turner AM. Interventions for the management and prevention of sarcopenia in the critically ill: A systematic review. J Crit Care. 2019;50:28795.

16. Wageck B, Nunes GS, Silva FL, Damasceno MC, de Noronha M. Application and effects of neuromuscular electrical stimulation in critically ill patients: systematic review. Med Intensiva. 2014;38(7):444-54.

17. Dirks ML, Hansen D, Van Assche A, Dendale P, Van Loon LJ. Neuromuscular electrical stimulation prevents muscle wasting in critically ill comatose patients. Clin Sci (Lond). 2015; 128(6):357-65.

18. Kho ME, Truong AD, Zanni JM, Ciesla ND, Brower RG, Palmer JB, et al. Neuromuscular electrical stimulation in mechanically ventilated patients: a randomized, sham-controlled pilot trial with blinded outcome assessment. J Crit Care. 2015;30(1):32-9.

19. Maffiuletti NA, Roig M, Karatzanos E, Nanas S. Neuromuscular electrical stimulation for preventing skeletal-muscle weakness and wasting in critically ill patients: a systematic review. BMC Med. 2013;11:137.

20. Segers J, Vanhorebeek I, Langer D, Charususin N, Wei W, Frickx B, et al. Early neuromuscular electrical stimulation reduces the loss of muscle mass in critically ill patients A within subject randomized controlled trial. J Crit Care. 2021;62:65-71.

21. Rodriguez PO, Setten M, Maskin LP, Bonelli I, Vidomlansky SR, Attie S, et al. Muscle weakness in septic patients requiring mechanical ventilation: protective effect of transcutaneous neuromuscular electrical stimulation. J Crit Care. 2012;27(3):319.e1-8.

22. Routsi C, Gerovasili V, Vasileiadis I, Karatzanos E, Pitsolis T, Tripodaki E, et al. Electrical muscle stimulation prevents critical illness polyneuromyopathy: a randomized parallel intervention trial. Crit Care. 2010;14(2):R74.

23. López-López L, Torres-Sánchez I, Rodríguez-Torres J, Cabrera-Martos I, Ortiz-Rubio A, Valenza MC. Does adding an integrated physical therapy and neuromuscular electrical stimulation therapy to standard rehabilitation improve functional outcome in elderly patients with pneumonia? A randomised controlled trial. Clin Rehabil. 2019;33(11):1757-66.

24. Liu M, Luo J, Zhou J, Zhu X. Intervention effect of neuromuscular electrical stimulation on ICU acquired weakness: A meta-analysis. Int J Nurs Sci. 2020;7(2):228-37.

25. Bernhardt J, Churilov L, Ellery F, Collier J, Chamberlain J, Langhorne P, et al. Prespecified dose-response analysis for A Very Early Rehabilitation Trial (AVERT). Neurology. 2016;86(23):2138-45.

26. Charlson ME, Pompei P, Ales KL, MacKenzie CR. A new method of classifying prognostic comorbidity in longitudinal studies: development and validation. J Chronic Dis. 1987;40(5):373-83.

27. Le Gall JR, Loirat P, Alperovitch A, Glaser P, Granthil C, Mathieu D, et al. A simplified acute physiology score for ICU patients. Crit Care Med. 1984;12(11):975-7. 
28. Vincent JL, Moreno R, Takala J, Willatts S, De Mendonça A, Bruining H, et al. The SOFA (Sepsis-related Organ Failure Assessment) score to describe organ dysfunction/failure. On behalf of the Working Group on Sepsis-Related Problems of the European Society of Intensive Care Medicine. Intensive Care Med. 1996;22(7):707-10.

29. Ely EW, Margolin R, Francis J, May L, Truman B, Dittus R, et al. Evaluation of delirium in critically ill patients: validation of the Confusion Assessment Method for the Intensive Care Unit (CAM-ICU). Crit Care Med. 2001;29(7):1370-9.

30. Dolan P. Modeling valuations for EuroQol health states. Med Care. 1997;35(11):1095108.

31. Ferreira PL, Ferreira LN, Pereira LN. [Contribution for the validation of the Portuguese version of EQ-5D]. Acta Med Port. 2013;26(6):664-75.

32. Silva VZMD, Araújo JA, Cipriano G, Pinedo M, Needham DM, Zanni JM, et al. Brazilian version of the Functional Status Score for the ICU: translation and cross-cultural adaptation. Rev Bras Ter Intensiva. 2017;29(1):34-8.

33. Parry SM, Denehy L, Beach LJ, Berney S, Williamson HC, Granger CL. Functional outcomes in ICU - what should we be using? - an observational study. Crit Care. 2015;19:127.

34. Thrush A, Rozek M, Dekerlegand JL. The clinical utility of the functional status score for the intensive care unit (FSS-ICU) at a long-term acute care hospital: a prospective cohort study. Phys Ther. 2012;92(12):1536-45.

35. Nordon-Craft A, Schenkman M, Edbrooke L, Malone DJ, Moss M, Denehy L. The physical function intensive care test: implementation in survivors of critical illness. Phys Ther. 2014;94(10):1499-507.

36. De Jonghe B, Bastuji-Garin S, Durand MC, Malissin I, Rodrigues P, Cerf C, et al. Respiratory weakness is associated with limb weakness and delayed weaning in critical illness. Crit Care Med. 2007;35(9):2007-15.

37. Silveira LTYD, Silva JMD, Soler JMP, Sun CYL, Tanaka C, Fu C. Assessing functional status after intensive care unit stay: the Barthel Index and the Katz Index. Int $\mathbf{J}$ Qual Health Care. 2018;30(4):265-70.

38. Leite MA, Osaku EF, Albert J, Costa CRLM, Garcia AM, Czapiesvski FDN, et al. Effects of Neuromuscular Electrical Stimulation of the Quadriceps and Diaphragm in Critically Ill Patients: A Pilot Study. Crit Care Res Pract. 2018;2018:4298583.

39. Gerovasili V, Stefanidis K, Vitzilaios K, Karatzanos E, Politis P, Koroneos A, et al. Electrical muscle stimulation preserves the muscle mass of critically ill patients: a randomized study. Crit Care. 2009;13(5):R161.

40. Wollersheim T, Grunow JJ, Carbon NM, Haas K, Malleike J, Ramme SF, et al. Muscle wasting and function after muscle activation and early protocol-based physiotherapy: an explorative trial. J Cachexia Sarcopenia Muscle. 2019;10(4):734-47.

41. Fossat G, Baudin F, Courtes L, Bobet S, Dupont A, Bretagnol A, et al. Effect of InBed Leg Cycling and Electrical Stimulation of the Quadriceps on Global Muscle Strength in Critically Ill Adults: A Randomized Clinical Trial. JAMA. 2018;320(4):368-78.

42. Segers J, Hermans G, Bruyninckx F, Meyfroidt G, Langer D, Gosselink R. Feasibility of neuromuscular electrical stimulation in critically ill patients. J Crit Care. 2014;29(6):10828.

43. Patsaki I, Gerovasili V, Sidiras G, Karatzanos E, Mitsiou G, Papadopoulos E, et al. Effect of neuromuscular stimulation and individualized rehabilitation on muscle strength in Intensive Care Unit survivors: A randomized trial. J Crit Care. 2017;40:76-82.

44. Zanotti E, Felicetti G, Maini M, Fracchia C. Peripheral muscle strength training in bed-bound patients with COPD receiving mechanical ventilation: effect of electrical stimulation. Chest. 2003;124(1):292-6. 
45. Dirks ML, Wall BT, van Loon LJC. Interventional strategies to combat muscle disuse atrophy in humans: focus on neuromuscular electrical stimulation and dietary protein. J Appl Physiol (1985). 2018;125(3):850-61. 


\section{FIGURE LEGENDS}

Figure 1: Flowchart of the study.

h: hours; ICU: intensive care unit; EM: early mobilization; NMES: neuromuscular electrical stimulation; FSS-ICU: functional status Scale in the intensive care unit; LL: lower limbs; FICF: free and informed consent form.

Figure 2: Boxplot of the functional status and muscle strength outcomes.

ICU: intensive care unit; EM: early mobilization; NMES: neuromuscular electrical stimulation; FSS-ICU: functional status scale in the intensive care unit; PFIT: physical function test in the intensive care unit; MRC-SS: Medical research council sum-score.

Figure 3: Kaplan-Meier curves to demonstrate the number of days until achieving motor milestones. EM: early mobilization; NMES: neuromuscular electrical stimulation; $n$ : sample size.

The number ' 1 ' at the $y$ axis means that the motor milestone was achieved, and the number ' 0 ' means it was not.

Variables are expressed as median and interquartile range. 
TABLES

Table 1: Baseline characteristics.

\begin{tabular}{lccc}
\hline Variables & EM & EM + NMES & $p$ Value \\
& $n=69$ & $n=70$ & 0.971 \\
Age & $50 \pm 18$ & $50 \pm 18$ & 0.112 \\
Memale & $42,03 \%$ & $28,57 \%$ & 0.924 \\
SAPS 3 & $57,97 \%$ & $71,43 \%$ & 0.642 \\
SOFA & $69,59 \pm 16,50$ & $69,36 \pm 12,78$ & 0.799 \\
CCI & $10,04 \pm 2,74$ & $9,81 \pm 3,04$ & 0.926 \\
BMI & $2,81 \pm 2,42$ & $2,72 \pm 2,40$ & 0.694 \\
Barthel index & $26,78 \pm 6,51$ & $26,63 \pm 6,28$ & \\
Diagnoses & $99,41 \pm 2,03$ & $99,57 \pm 1,65$ & \\
Stroke & & & \\
Traumatic brain injury & $15(21 \%)$ & $22(31 \%)$ & 0.827 \\
Post-surgical & $18(26 \%)$ & $19(27 \%)$ & \\
Respiratory failure & $43(62 \%)$ & $37(52 \%)$ & \\
Sepsis & $36(52 \%)$ & $35(50 \%)$ & \\
Others & $34(49 \%)$ & $30(43 \%)$ & \\
\hline
\end{tabular}

EM: Early mobilization; NMES: Neuromuscular electrical stimulation; SAPS 3: Simplified Acute Physiology Score 3; SOFA: Sequential Organ Failure Assessment; CCI: Charlson's Comorbidity Index; BMI: Body mass index.

Categorical variables are expressed as percentage. Continuous variables are expressed as mean \pm standard deviation. 
Table 2: Primary and secondary outcomes. Sample sizes ( $n), p$ value and effect size.

\begin{tabular}{|c|c|c|c|c|c|c|}
\hline Outcome & EM & $\mathrm{N}$ & EM+NMES & $\mathrm{N}$ & $p$ Value & $\begin{array}{r}\text { Effect } \\
\text { size }\end{array}$ \\
\hline \multicolumn{7}{|l|}{ At the first day awake } \\
\hline FSS-ICU, score & $15.2 \pm 8.4$ & 23 & $21.2 \pm 6.8$ & 20 & .015 & .77 \\
\hline PFIT, score & $5.8 \pm 2.8$ & 22 & $8.1 \pm 2.4$ & 18 & .018 & .88 \\
\hline MRC-SS, score & $42(33-54)$ & 18 & $54(47-60)$ & 15 & .011 & .65 \\
\hline MRC-SS LL, score & $22(18-27)$ & 18 & $30(24-30)$ & 15 & .005 & 1.02 \\
\hline \multicolumn{7}{|l|}{ At ICU discharge } \\
\hline FSS-ICU, score & $18.9 \pm 8.6$ & 26 & $26.3 \pm 7.8$ & 21 & .003 & .89 \\
\hline PFIT, score & $7(5-9)$ & 22 & $11(10-12)$ & 18 & .001 & .20 \\
\hline MRC-SS, score & $50(39-56)$ & 22 & $58.5(55-60)$ & 18 & .001 & .65 \\
\hline MRC-SS LL, score & $26.5(20-28)$ & 22 & $30(28-30)$ & 18 & .001 & .59 \\
\hline Barthel index & $15(4-51)$ & 38 & $37(0-61)$ & 34 & .202 & .41 \\
\hline EQ-5D-3L index & $0 \pm 0.25$ & 25 & $0.11 \pm 0.29$ & 18 & .147 & .41 \\
\hline EQ VAS & $70(52-80)$ & 25 & $79(50-80)$ & 18 & .225 & .31 \\
\hline \multicolumn{7}{|l|}{ At Hospital discharge } \\
\hline FSS-ICU, score & $24.5 \pm 8.7$ & 26 & $29 \pm 8.8$ & 24 & .014 & .69 \\
\hline PFIT, score & $9(6-11)$ & 26 & $11(10-12)$ & 21 & .005 & .50 \\
\hline MRC-SS, score & $52(49-59)$ & 23 & $59(56-60)$ & 22 & .010 & .90 \\
\hline MRC-SS LL, score & $28(23-30)$ & 23 & $30(29-30)$ & 22 & .011 & .39 \\
\hline Barthel index & $73(15-80)$ & 36 & $70(25-95)$ & 33 & .085 & .44 \\
\hline EQ-5D-3L index & $0.11 \pm 0.35$ & 26 & $0.22 \pm 0.29$ & 21 & .319 & .33 \\
\hline EQ VAS & $77(59-86)$ & 26 & $80(70-92)$ & 21 & .086 & .09 \\
\hline ICU LOS, days & $15(7-23)$ & 69 & $11(6-16)$ & 70 & .061 & .29 \\
\hline Hospital LOS, days & $30(12-40)$ & 69 & $18.5(10-29)$ & 70 & .048 & .47 \\
\hline Days of MV & $12(7-16)$ & 69 & $9(6-13)$ & 70 & .135 & .35 \\
\hline $\begin{array}{l}\text { Incidence of ICU-AW*, n } \\
\text { (\%) }\end{array}$ & $10(45)$ & 22 & $2(11)$ & 18 & .035 & - \\
\hline
\end{tabular}

EM: early mobilization; NMES: neuromuscular electrical stimulation ; ICU: intensive care unit; LOS: length of stay; MV: mechanical ventilation; ICU-AW: ICU acquired weakness; FSS-ICU: functional status scale in the intensive care unit; PFIT: physical function test in the intensive care unit; MRC-SS: medical research council sum-score; LL: lower limbs; EQ-5D-3L: Euro Quality of life-5 dimensions and 3 levels questionnaire; EQ VAS: euro quality visual analogue scale. Variables are expressed as median (interquartile range) or mean \pm standard deviation. ${ }^{*}$ ICU-AW detected with MRC sum $<48$ at ICU discharge. 


\section{FIGURES}

\section{Figure 1}

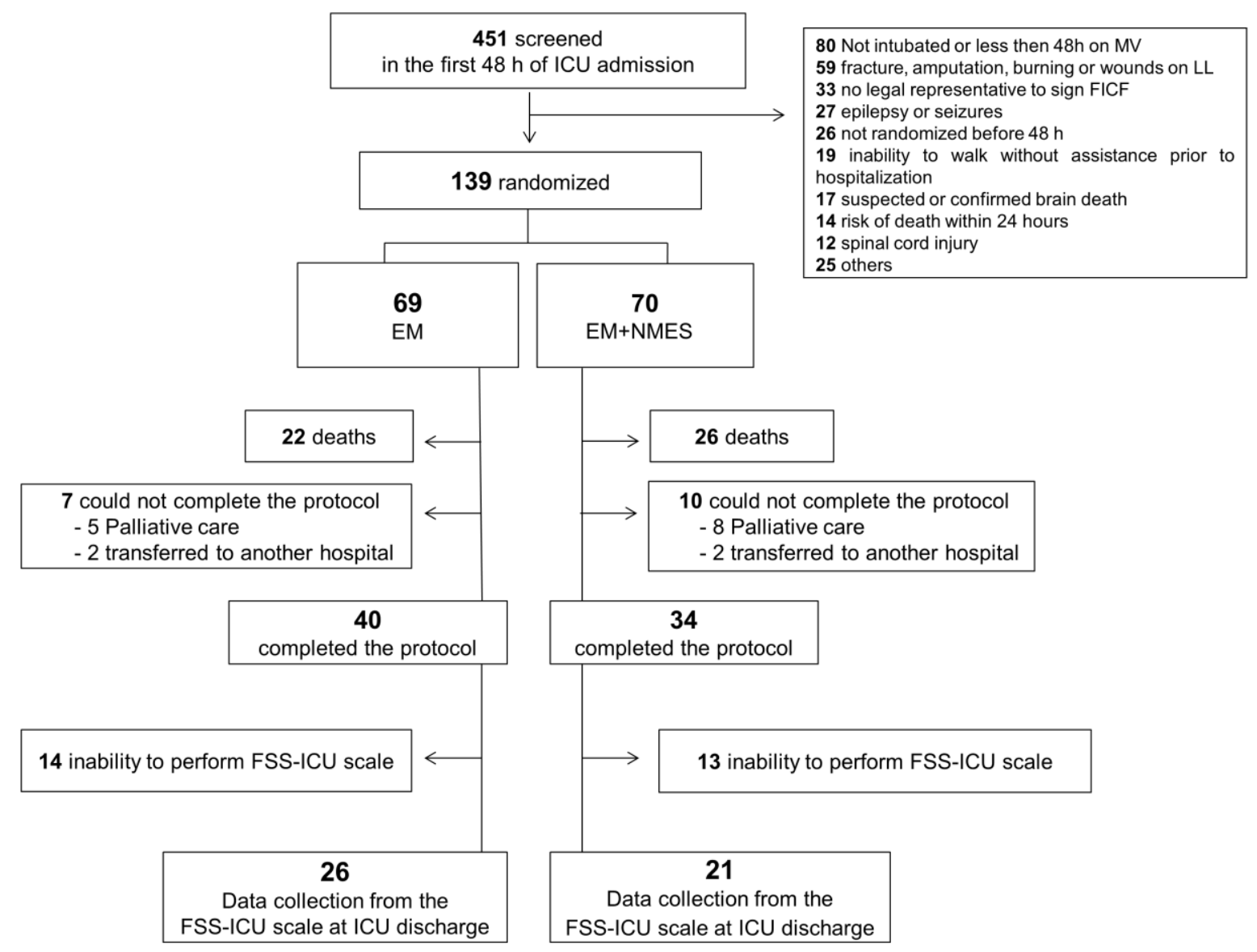


Figure 2
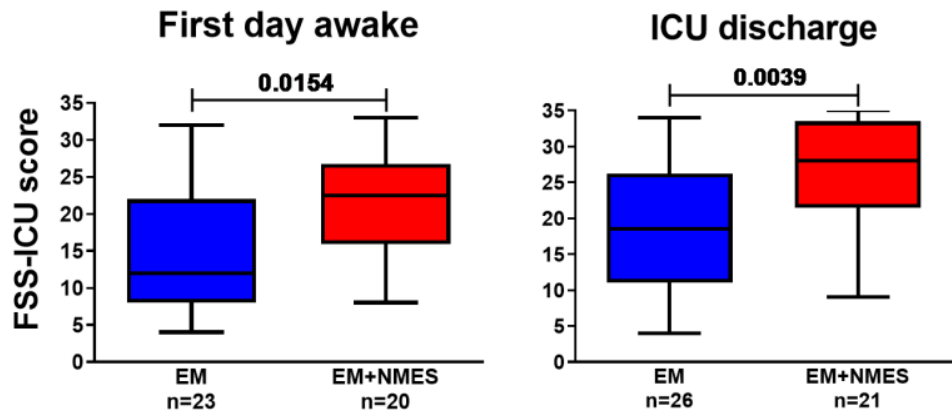

Hospital discharge
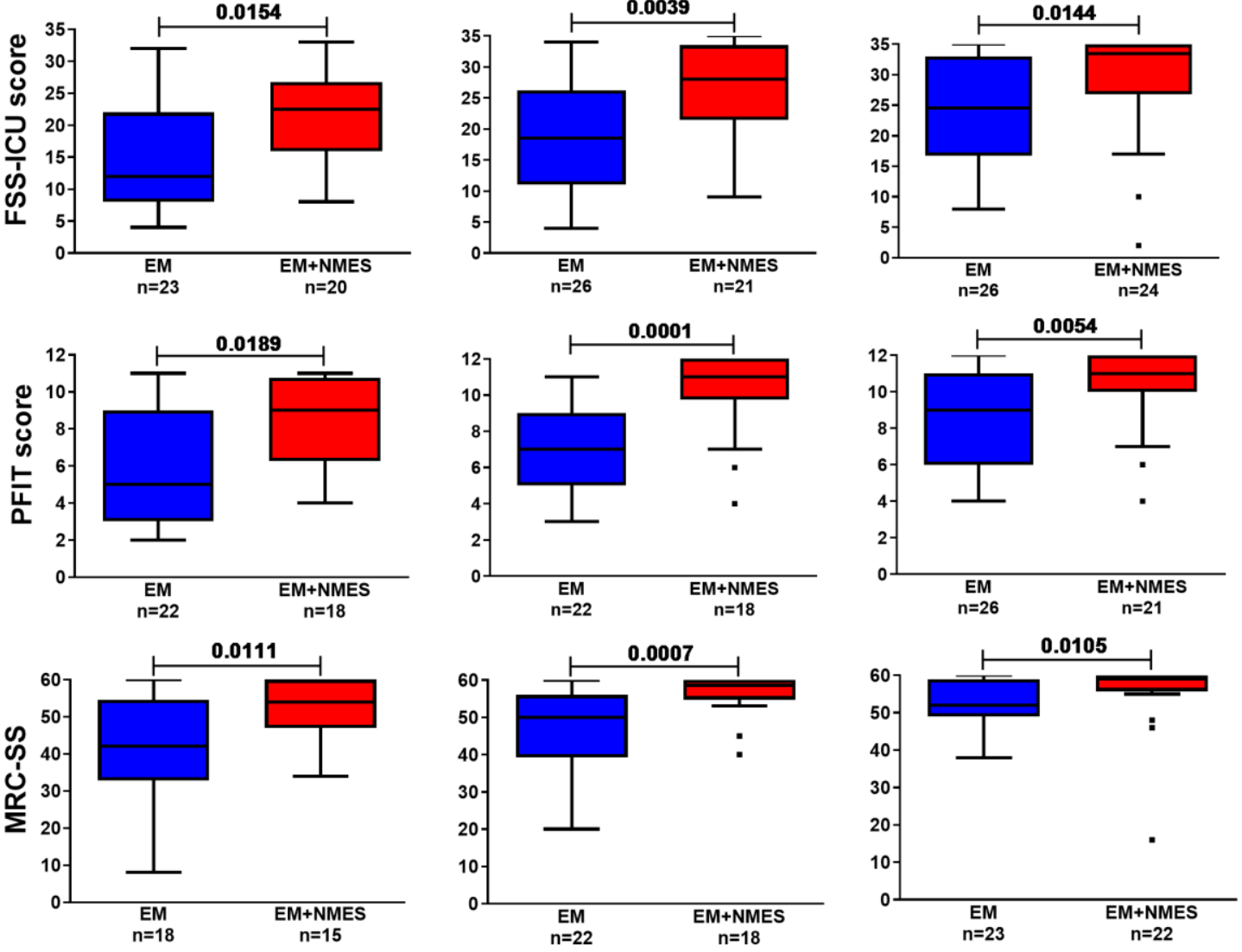
Figure 3

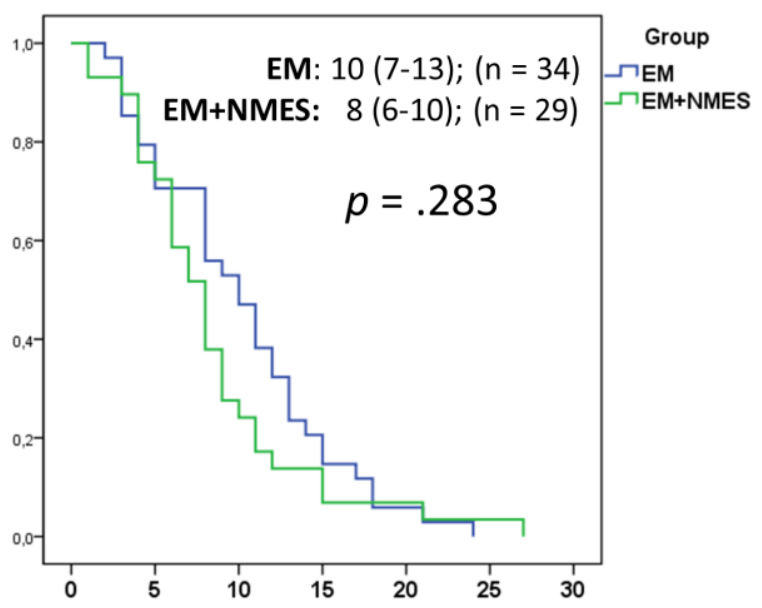

Number of days until sitting on the edge of the bed

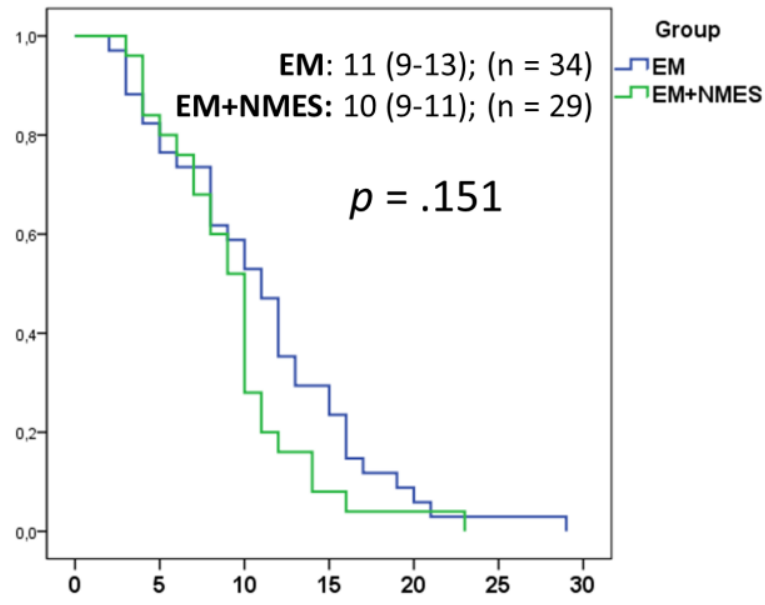

Number of days until sitting on the armchair
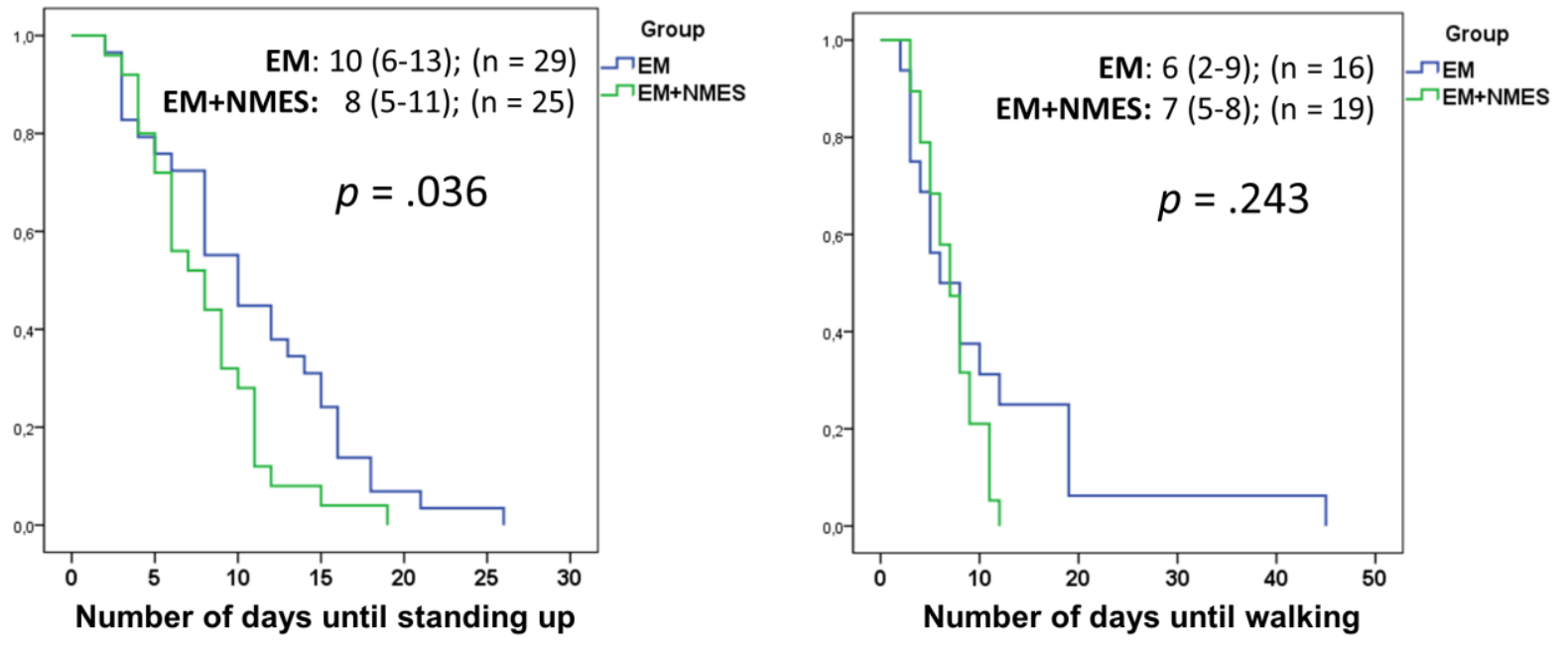